\title{
Microwave-assisted catalyst-free synthesis of tetrasubtituted pyrroles using dialkyl acetylenedicarboxylates and monophenacylaniline
}

\author{
Arumugam Mariappan, ${ }^{\dagger}$ Kandasamy Rajaguru, ${ }^{\dagger}$ Shanmugam Muthusubramanian, ${ }^{\dagger, *}$ \\ and Nattamai Bhuvanesh". \\ ${ }^{\dagger}$ Department of Organic Chemistry, School of Chemistry, Madurai Kamaraj University, \\ Madurai - 625 021, India. \\ ${ }^{\#} X$-ray Diffraction Laboratory, Department of Chemistry, Texas A \& M University, \\ College Station, TX 77842, USA. \\ e-mail:muthumanian2001@yahoo.com \\ *Corresponding author \\ Table of contents
}

Page No

Experimental section 3

Characterization data for compounds 3a-3r 4-13

${ }^{1} \mathrm{H} \&{ }^{13} \mathrm{C}$ NMR spectra of compound $\mathbf{3 a}$ 14

${ }^{1} \mathrm{H},{ }^{13} \mathrm{C}$ NMR \& Mass spectra of compound $\mathbf{3 b}$ 16

${ }^{1} \mathrm{H} \&{ }^{13} \mathrm{C}$ NMR spectra of compound $\mathbf{3 c}$ 19

${ }^{1} \mathrm{H},{ }^{13} \mathrm{C}$ NMR \& Mass spectra of compound 3d 21

${ }^{1} \mathrm{H} \&{ }^{13} \mathrm{C}$ NMR spectra of compound $\mathbf{3 e}$ 24

${ }^{1} \mathrm{H} \&{ }^{13} \mathrm{C}$ NMR spectra of compound $\mathbf{3 f}$ 26

${ }^{1} \mathrm{H} \&{ }^{13} \mathrm{C}$ NMR spectra of compound $\mathbf{3 g}$ 28

${ }^{1} \mathrm{H},{ }^{13} \mathrm{C}$ NMR \& Mass spectra of compound $\mathbf{3 h}$ 30

${ }^{1} \mathrm{H} \&{ }^{13} \mathrm{C}$ NMR spectra of compound $\mathbf{3 i}$ 33

${ }^{1} \mathrm{H} \&{ }^{13} \mathrm{C}$ NMR spectra of compound $\mathbf{3 j}$ 35

${ }^{1} \mathrm{H} \&{ }^{13} \mathrm{C}$ NMR spectra of compound $\mathbf{3 k}$ 37

${ }^{1} \mathrm{H} \&{ }^{13} \mathrm{C}$ NMR spectra of compound $3 \mathrm{I}$ 39

${ }^{1} \mathrm{H} \&{ }^{13} \mathrm{C}$ NMR spectra of compound $\mathbf{3 m}$ 41

${ }^{1} \mathrm{H} \&{ }^{13} \mathrm{C}$ NMR spectra of compound $\mathbf{3 n}$ 43

${ }^{1} \mathrm{H} \&{ }^{13} \mathrm{C}$ NMR spectra of compound 30 45

${ }^{1} \mathrm{H},{ }^{13} \mathrm{C}$ NMR \& Mass spectra of compound 3p 47

${ }^{1} \mathrm{H},{ }^{13} \mathrm{C}$ NMR \& Mass spectra of compound 3q 50

${ }^{1} \mathrm{H} \&{ }^{13} \mathrm{C}$ NMR spectra of compound $\mathbf{3 r}$ 53

${ }^{1} \mathrm{H} \&{ }^{13} \mathrm{C}$ NMR spectra of compound $3 \mathrm{~s}$ 55 
${ }^{1} \mathrm{H} \&{ }^{13} \mathrm{C}$ NMR spectra of compound $\mathbf{3 t}$

${ }^{1} \mathrm{H} \&{ }^{13} \mathrm{C}$ NMR spectra of compound $3 \mathbf{u}$ 59

References

61 


\section{Experimental Section}

\section{General Remarks:}

All solvents were purchased from commercial sources and used without further purification. The melting points were measured in open capillary tubes and are uncorrected. A CEM Discover microwave synthesizer (Model No: 908010) operating at 180/264 V and 50/60 Hz with microwave power maximum level of $300 \mathrm{~W}$ and microwave frequency of $2455 \mathrm{MHz}$ was employed for the microwave-assisted experiments. Nuclear Magnetic Resonance $\left({ }^{1} \mathrm{H}\right.$ and ${ }^{13} \mathrm{C}$ NMR) spectra were recorded on a $300 \mathrm{MHz}$ spectrometer in $\mathrm{CDCl}_{3}$ using TMS as an internal standard. Chemical shifts are reported in parts per million $(\delta)$, coupling constants $(J$ values) are reported in Hertz $(\mathrm{Hz})$ and spin multiplicities are indicated by the following symbols: s (singlet), $d$ (doublet), t (triplet), q (quatret), sept (septet), m (multiplet). ${ }^{13} \mathrm{C}$ NMR spectra were routinely run with broadband decoupling. Elemental analyses were performed on a Perkin Elmer 2400Series II Elemental CHNS analyzer.

\section{General procedure for the synthesis of Dimethyl 1,4-diphenyl-1H-pyrrole-2,3-dicarboxylate 3}

A mixture of substituted monophenacylaniline 1 (1 mmol), dialkyl acetylenedicarboxylate $2(1.1 \mathrm{mmol})$ in DMF (1 mL) was sealed and subjected to microwave irradiation programmed at $110{ }^{\circ} \mathrm{C}$ and $110 \mathrm{~W}$ for 10 minutes. The completion of the reaction was monitored by TLC. The reaction mixture was extracted with ethyl acetate. The organic layer was washed with water and brine, dried over anhydrous sodium sulfate and concentrated in vacuum. Crude product was purified by column chromatography using petroleum ether - ethyl acetate (5: 95) as the eluent to get 3 . 


\section{Characterization data for compounds (3a-3r)}

\section{Diethyl 1,4-bis(4-chlorophenyl)-1H-pyrrole-2,3-dicarboxylate (3c)}

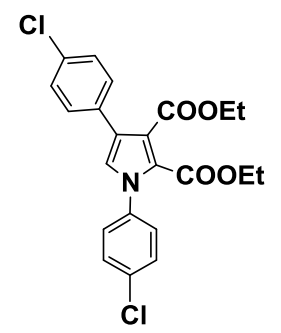

Yellow viscous liquid; IR (neat): 1728, 1698, 1590, $1453 \mathrm{~cm}^{-1} .{ }^{1} \mathrm{H}$ NMR $\left(300 \mathrm{MHz}, \mathrm{CDCl}_{3}\right) \delta 7.44$ - $7.34(\mathrm{~m}, 6 \mathrm{H}), 7.31-7.27(\mathrm{~m}, 2 \mathrm{H}), 6.94(\mathrm{~s}, 1 \mathrm{H}), 4.32(\mathrm{q}, J=7.1 \mathrm{~Hz}, 2 \mathrm{H}), 4.18(\mathrm{q}, J=7.1 \mathrm{~Hz}, 2 \mathrm{H})$, $1.30(\mathrm{t}, J=7.1 \mathrm{~Hz}, 3 \mathrm{H}), 1.19(\mathrm{t}, J=7.1 \mathrm{~Hz}, 3 \mathrm{H}) .{ }^{13} \mathrm{C} \mathrm{NMR}\left(75 \mathrm{MHz}, \mathrm{CDCl}_{3}\right) \delta 165.6,159.7$, 137.9, 134.5, 133.2, 131.6, 129.1, 129.1, 128.7, 127.5, 125.4, 124.0, 123.8, 122.1, 61.3, 61.0, 14.0, 13.9. Anal. Calcd for $\mathrm{C}_{22} \mathrm{H}_{19} \mathrm{Cl}_{2} \mathrm{NO}_{4}$ : C, 61.13; H, 4.43; N, $3.24 \%$. Found: C, 61.08; H, 4.47; N, $3.30 \%$.

Diethyl 4-(4-chlorophenyl)-1-(p-tolyl)-1H-pyrrole-2,3-dicarboxylate (3d)

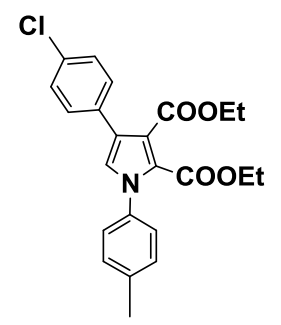

Yellow solid; mp $97-100{ }^{\circ} \mathrm{C}$; IR (neat): 1730, 1700, 1588, $1454 \mathrm{~cm}^{-1} .{ }^{1} \mathrm{H}$ NMR $(300 \mathrm{MHz}$, $\left.\mathrm{CDCl}_{3}\right) \delta 7.40-7.31(\mathrm{~m}, 4 \mathrm{H}), 7.27-7.21(\mathrm{~m}, 4 \mathrm{H}), 6.94(\mathrm{~s}, 1 \mathrm{H}), 4.31(\mathrm{q}, J=7.1 \mathrm{~Hz}, 2 \mathrm{H}), 4.18(\mathrm{q}$, $J=7.1 \mathrm{~Hz}, 2 \mathrm{H}), 2.41(\mathrm{~s}, 3 \mathrm{H}), 1.29(\mathrm{t}, J=7.1 \mathrm{~Hz}, 3 \mathrm{H}), 1.18(\mathrm{t}, J=7.1 \mathrm{~Hz}, 3 \mathrm{H}) .{ }^{13} \mathrm{C} \mathrm{NMR}(75 \mathrm{MHz}$, $\left.\mathrm{CDCl}_{3}\right) \delta 165.9,159.9,138.5,136.8,132.9,131.8,129.4,129.1,128.6,125.8,125.5,123.9,123.5$, 
121.2, 61.3, 60.9, 21.1, 14.0, 13.9. Anal. Calcd for $\mathrm{C}_{23} \mathrm{H}_{22} \mathrm{ClNO}_{4}$ : C, 67.07; H, 5.38; N, $3.40 \%$.

Found: C, 67.09; H, 5.33; N, $3.43 \%$. ESI-MS m/z calcd $[\mathrm{M}+\mathrm{H}]^{+}$412.12, found 412.11.

\section{Diethyl 4-(4-bromophenyl)-1-(p-tolyl)-1H-pyrrole-2,3-dicarboxylate (3e)}

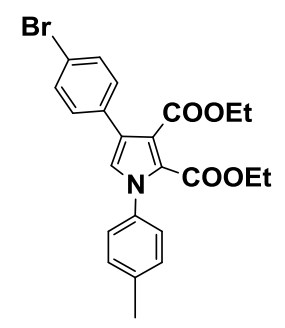

Brown viscous liquid; IR (neat): $3123,1731,1703,1592,1446 \mathrm{~cm}^{-1} .{ }^{1} \mathrm{H} \mathrm{NMR}\left(300 \mathrm{MHz}, \mathrm{CDCl}_{3}\right)$ $\delta 7.48(\mathrm{~d}, J=8.6 \mathrm{~Hz}, 2 \mathrm{H}), 7.32(\mathrm{~d}, J=8.6 \mathrm{~Hz}, 2 \mathrm{H}), 7.24-7.21(\mathrm{~m}, 4 \mathrm{H}), 6.94(\mathrm{~s}, 1 \mathrm{H}), 4.31(\mathrm{q}, J=$ $7.1 \mathrm{~Hz}, 2 \mathrm{H}), 4.17(\mathrm{q}, J=7.1 \mathrm{~Hz}, 2 \mathrm{H}), 2.41(\mathrm{~s}, 3 \mathrm{H}), 1.29(\mathrm{t}, J=7.2 \mathrm{~Hz}, 3 \mathrm{H}), 1.17(\mathrm{t}, J=7.1 \mathrm{~Hz}$, $3 \mathrm{H}) .{ }^{13} \mathrm{C} \mathrm{NMR}\left(75 \mathrm{MHz}, \mathrm{CDCl}_{3}\right) \delta 166.4,160.2,139.0,137.2,132.7,131.9,{ }^{*} 129.9,129.9,126.3$, 125.9, 123.8, 121.6, 121.5, 61.7, 61.3, 21.6, 14.5, 14.3. Anal. Calcd for $\mathrm{C}_{23} \mathrm{H}_{22} \mathrm{BrNO}_{4}: \mathrm{C}, 60.54 ; \mathrm{H}$, 4.86; N, $3.07 \%$. Found: C, 60.51; H, 4.91; N, $3.05 \% . *($ Two carbon signals merged here)

\section{Diethyl 1-(4-chlorophenyl)-4-(p-tolyl)-1H-pyrrole-2,3-dicarboxylate (3f)}

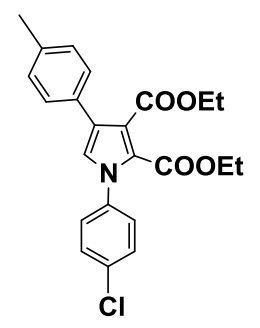

White solid; mp $99-101{ }^{\circ} \mathrm{C}$; IR (neat): 3130, 1730, 1701, 1511, $1418 \mathrm{~cm}^{-1} .{ }^{1} \mathrm{H}$ NMR $(300 \mathrm{MHz}$, $\left.\mathrm{CDCl}_{3}\right) \delta 7.42(\mathrm{~d}, J=8.7 \mathrm{~Hz}, 2 \mathrm{H}), 7.35-7.27(\mathrm{~m}, 4 \mathrm{H}), 7.18(\mathrm{~d}, J=7.8 \mathrm{~Hz}, 2 \mathrm{H}), 6.94(\mathrm{~s}, 1 \mathrm{H}), 4.2$ $(\mathrm{q}, J=7.1 \mathrm{~Hz}, 2 \mathrm{H}), 4.2(\mathrm{q}, J=7.1 \mathrm{~Hz}, 2 \mathrm{H}), 2.36(\mathrm{~s}, 3 \mathrm{H}), 1.30(\mathrm{t}, J=7.1 \mathrm{~Hz}, 3 \mathrm{H}), 1.19(\mathrm{t}, J=7.1$ $\mathrm{Hz}, 3 \mathrm{H}) .{ }^{13} \mathrm{C} \mathrm{NMR}\left(75 \mathrm{MHz}, \mathrm{CDCl}_{3}\right) \delta 165.8,159.7,138.1,136.9,134.3,129.9,129.2,129.0$, 
127.6, 127.5, 125.6, 124.9, 122.7, 122.6, 61.4, 60.8, 21.1, 14.0, 13.9. Anal. Calcd for $\mathrm{C}_{23} \mathrm{H}_{22} \mathrm{ClNO}_{4}$ : C, 67.07; H, 5.38; N, $3.40 \%$. Found: C, 67.09; H, 5.41; N, $3.36 \%$.

\section{Diethyl 1-(4-chlorophenyl)-4-(4-methoxyphenyl)-1H-pyrrole-2,3-dicarboxylate (3g)}

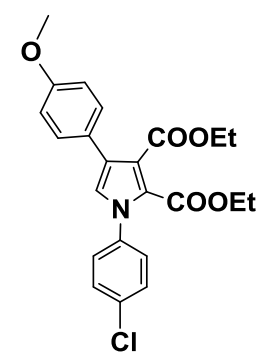

Yellow viscous liquid; IR (neat): 1707, 1611, 1586, $1450 \mathrm{~cm}^{-1} .{ }^{1} \mathrm{H}$ NMR $\left(300 \mathrm{MHz}, \mathrm{CDCl}_{3}\right) \delta 7.44$ $-7.36(\mathrm{~m}, 4 \mathrm{H}), 7.30-7.24(\mathrm{~m}, 2 \mathrm{H}), 6.91(\mathrm{~m}, 3 \mathrm{H}), * 4.32(\mathrm{q}, J=7.1 \mathrm{~Hz}, 2 \mathrm{H}), 4.17(\mathrm{q}, J=7.1 \mathrm{~Hz}$, 2H), $3.83(\mathrm{~s}, 3 \mathrm{H}), 1.30(\mathrm{t}, J=7.1 \mathrm{~Hz}, 3 \mathrm{H}), 1.19(\mathrm{t}, J=7.1 \mathrm{~Hz}, 3 \mathrm{H}) .{ }^{13} \mathrm{C} \mathrm{NMR}\left(75 \mathrm{MHz}, \mathrm{CDCl}_{3}\right) \delta$ $166.1,159.6,158.8,138.0,134.2,128.9, * 128.8,127.5,125.3,124.6,122.7,122.4,113.9,61.3$, 60.8, 55.1, 14.0, 13.8. Anal. Calcd for $\mathrm{C}_{23} \mathrm{H}_{22} \mathrm{ClNO}_{5}$ : C, 64.56; H, 5.18; N, $3.27 \%$. Found: $\mathrm{C}$, 64.60; H, 5.21; N, $3.23 \%$. *(Two carbon signals merged here).

\section{Diethyl 4-(4-methoxyphenyl)-1-(p-tolyl)-1H-pyrrole-2,3-dicarboxylate (3h)}

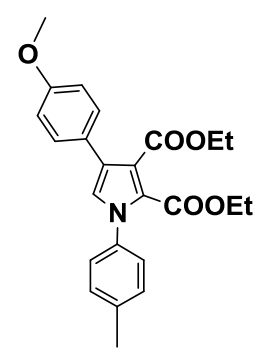

Pale brown solid; mp $62-64{ }^{\circ} \mathrm{C}$; IR (neat): $1706,1608,1579 \mathrm{~cm}^{-1} .{ }^{1} \mathrm{H}$ NMR $\left(300 \mathrm{MHz}, \mathrm{CDCl}_{3}\right) \delta$ $7.38(\mathrm{~d}, J=8.7 \mathrm{~Hz}, 2 \mathrm{H}), 7.21-7.24(\mathrm{~m}, 4 \mathrm{H}), 6.92-6.89(\mathrm{~m}, 3 \mathrm{H}), 4.31(\mathrm{q}, J=7.1 \mathrm{~Hz}, 2 \mathrm{H}), 4.17$ (q, $J=7.1 \mathrm{~Hz}, 2 \mathrm{H}), 3.83(\mathrm{~s}, 3 \mathrm{H}), 2.41(\mathrm{~s}, 3 \mathrm{H}), 1.30(\mathrm{t}, J=7.1 \mathrm{~Hz}, 3 \mathrm{H}), 1.19(\mathrm{t}, J=7.1 \mathrm{~Hz}, 3 \mathrm{H}) .{ }^{13} \mathrm{C}$ 
NMR $\left(75 \mathrm{MHz}, \mathrm{CDCl}_{3}\right) \delta 166.3,159.9,158.7,138.3,137.0,129.3,128.9,125.9,125.7,125.4$, 124.3, 123.1, 121.5, 113.8, 61.2, 60.7, 55.2, 21.1, 14.0, 13.9. Anal. Calcd for $\mathrm{C}_{24} \mathrm{H}_{25} \mathrm{NO}_{5}: \mathrm{C}$, 70.75; H, 6.18; N, $3.44 \%$. Found: C, 70.72; H, 6.23; N, $3.47 \%$.

Diethyl 4-(4-nitrophenyl)-1-(p-tolyl)-1H-pyrrole-2,3-dicarboxylate (3i)

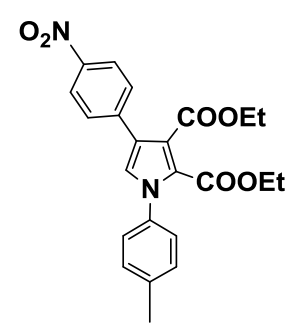

Yellow solid; mp $109-112{ }^{\circ} \mathrm{C}$; IR (neat):1721, 1595, 1513, 1338, $1225 \mathrm{~cm}^{-1} .{ }^{1} \mathrm{H}$ NMR $(300 \mathrm{MHz}$, $\left.\mathrm{CDCl}_{3}\right) \delta 8.23(\mathrm{~d}, J=8.8 \mathrm{~Hz}, 2 \mathrm{H}), 7.61(\mathrm{~d}, J=8.8 \mathrm{~Hz}, 2 \mathrm{H}), 7.29-7.22(\mathrm{~m}, 4 \mathrm{H}), 7.06(\mathrm{~s}, 1 \mathrm{H})$, $4.33(\mathrm{q}, J=7.1 \mathrm{~Hz}, 2 \mathrm{H}), 4.19(\mathrm{q}, J=7.1 \mathrm{~Hz}, 2 \mathrm{H}), 2.43(\mathrm{~s}, 3 \mathrm{H}), 1.31(\mathrm{t}, J=7.1 \mathrm{~Hz}, 3 \mathrm{H}), 1.20(\mathrm{t}, J=$

$7.1 \mathrm{~Hz}, 3 \mathrm{H}) .{ }^{13} \mathrm{C} \mathrm{NMR}\left(75 \mathrm{MHz}, \mathrm{CDCl}_{3}\right) \delta 165.3,159.9,146.7,140.3,138.9,136.6,130.2,129.6$, 128.4, 125.9, 125.8, 123.7, 122.7, 120.7, 61.4, 61.1, 21.1, 14.0, 13.8. Anal. Calcd for $\mathrm{C}_{23} \mathrm{H}_{22} \mathrm{~N}_{2} \mathrm{O}_{6}$ : C, 65.40; H, 5.25; N, $6.63 \%$. Found: C, 65.43; H, 5.20; N, $6.69 \%$.

\section{Diethyl 1-(2-isopropylphenyl)-4-phenyl-1H-pyrrole-2,3-dicarboxylate (3j)}

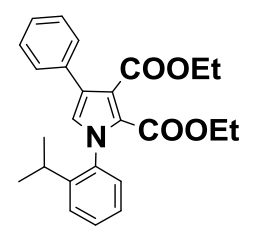

White solid; mp $71-73^{\circ} \mathrm{C}$; IR (neat): 3066, 2959, 1708, 1602, $1546 \mathrm{~cm}^{-1} .{ }^{1} \mathrm{H}$ NMR (300 MHz, $\left.\mathrm{CDCl}_{3}\right) \delta 7.50-7.47(\mathrm{~m}, 4 \mathrm{H}), 7.41-7.36(\mathrm{~m}, 2 \mathrm{H}), 7.32-7.20(\mathrm{~m}, 3 \mathrm{H}), 6.91(\mathrm{~s}, 1 \mathrm{H}), 4.36(\mathrm{q}, J$ $=7.2 \mathrm{~Hz}, 2 \mathrm{H}), 4.10(\mathrm{q}, J=7.1,1 \mathrm{H}), 4.09(\mathrm{q}, J=7.1,1 \mathrm{H}), 2.70(\mathrm{sept}, J=6 \mathrm{~Hz}, 1 \mathrm{H}), 1.32(\mathrm{t}, J=$ $7.1 \mathrm{~Hz}, 3 \mathrm{H}), 1.19(\mathrm{~d}, J=6.0 \mathrm{~Hz}, 3 \mathrm{H}), 1.16(\mathrm{~d}, J=6.0 \mathrm{~Hz}, 3 \mathrm{H}), 1.07(\mathrm{t}, J=7.1 \mathrm{~Hz}, 3 \mathrm{H}) .{ }^{13} \mathrm{C} \mathrm{NMR}$ 
$\left(75 \mathrm{MHz}, \mathrm{CDCl}_{3}\right) \delta 166.4,159.4,145.8,137.6,133.3,129.3,128.5,127.6,127.5,126.9,126.2$, 125.9, 125.8, 124.4, 123.9, 121.6, 61.3, 60.5, 27.8, 24.7, 23.1, 14.0, 13.7. Anal. Calcd for $\mathrm{C}_{25} \mathrm{H}_{27} \mathrm{NO}_{4}$ : C, 74.05; H, 6.71; N, $3.45 \%$. Found: C, 74.01; H, 6.76; N, 3.48

Diethyl 4-(4-chlorophenyl)-1-(3-methoxyphenyl)-1H-pyrrole-2,3-dicarboxylate (3k)

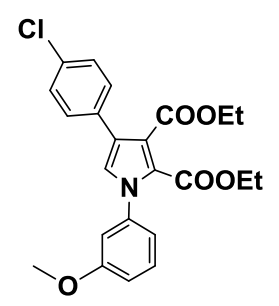

Brown viscous liquid; IR (neat): 1732, 1706, 1612, $1548 \mathrm{~cm}^{-1} .{ }^{1} \mathrm{H}$ NMR $\left(300 \mathrm{MHz}, \mathrm{CDCl}_{3}\right) \delta 7.40$ $-7.32(\mathrm{~m}, 5 \mathrm{H}), 6.99-6.92(\mathrm{~m}, 3 \mathrm{H}),{ }^{*} 6.89(\mathrm{~m}, 1 \mathrm{H}), 4.31(\mathrm{q}, J=7.1 \mathrm{~Hz}, 2 \mathrm{H}), 4.18(\mathrm{q}, J=7.1 \mathrm{~Hz}$, 2H), $3.82(\mathrm{~s}, 3 \mathrm{H}), 1.29(\mathrm{t}, J=7.1 \mathrm{~Hz}, 3 \mathrm{H}), 1.17(\mathrm{t}, J=7.1 \mathrm{~Hz}, 3 \mathrm{H}) .{ }^{13} \mathrm{C} \mathrm{NMR}\left(75 \mathrm{MHz}, \mathrm{CDCl}_{3}\right) \delta$ $165.7,159.9,159.8,140.3,133.0,131.7,129.6,129.2,128.6,125.3,124.2,123.7,121.1,118.2$, 114.2, 111.9, 61.3, 61.0, 55.4, 14.0, 13.8. Anal. Calcd for $\mathrm{C}_{23} \mathrm{H}_{22} \mathrm{ClNO}_{5}: \mathrm{C}, 64.56 ; \mathrm{H}, 5.18 ; \mathrm{N}$, 3.27\%. Found: C, 64.61; H, 5.21; N, $3.23 \%$.

Diethyl 4-(4-chlorophenyl)-1-(naphthalen-1-yl)-1H-pyrrole-2,3-dicarboxylate (3I)

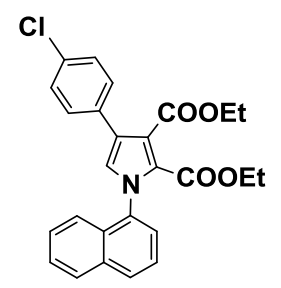

Brown viscous liquid; IR (neat): IR (neat): 1730, 1709, 1593, $1450 \mathrm{~cm}^{-1} .{ }^{1} \mathrm{H}$ NMR $(300 \mathrm{MHz}$, $\left.\mathrm{CDCl}_{3}\right) \delta 7.95(\mathrm{~m}, 2 \mathrm{H}), 7.56-7.47(\mathrm{~m}, 5 \mathrm{H}), 7.45-7.40(\mathrm{~m}, 2 \mathrm{H}), 7.35(\mathrm{~d}, J=8.5 \mathrm{~Hz}, 2 \mathrm{H}), 7.05$ $(\mathrm{s}, 1 \mathrm{H}), 4.37(\mathrm{q}, J=7.1 \mathrm{~Hz}, 2 \mathrm{H}), 3.95(\mathrm{q}, J=6 \mathrm{~Hz}, 1 \mathrm{H}), 3.94(\mathrm{q}, J=6 \mathrm{~Hz}, 1 \mathrm{H}), 1.34(\mathrm{~d}, J=7.1 \mathrm{~Hz}$, 
$3 \mathrm{H}), 0.85(\mathrm{t}, J=7.1 \mathrm{~Hz}, 3 \mathrm{H}) .{ }^{13} \mathrm{C} \mathrm{NMR}\left(75 \mathrm{MHz}, \mathrm{CDCl}_{3}\right) \delta 166.1,159.3,136.5,133.6,133.2$, $131.8,130.6,129.3,129.0,128.7,128.1,127.5,126.7,126.4,125.1,124.9,124.6,123.6,122.5$, 121.8, 61.4, 60.3, 14.1, 13.2. Anal. Calcd for $\mathrm{C}_{26} \mathrm{H}_{22} \mathrm{ClNO}_{4}$ : C, 69.72; H, 4.95; N, $3.13 \%$. Found: C, 69.76; H, 4.90; N, $3.10 \%$.

\section{Diethyl 4-(naphthalen-2-yl)-1-(p-tolyl)-1H-pyrrole-2,3-dicarboxylate (3m)}

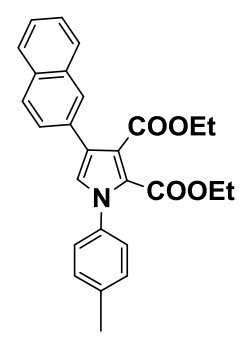

Pale yellow solid; mp $89-92{ }^{\circ} \mathrm{C}$; IR (neat): 1728, 1704, 1625, $1514 \mathrm{~cm}^{-1} .{ }^{1} \mathrm{H}$ NMR $(300 \mathrm{MHz}$, $\left.\mathrm{CDCl}_{3}\right) \delta 7.92(\mathrm{~s}, 1 \mathrm{H}), 7.85-7.81(\mathrm{~m}, 3 \mathrm{H}), 7.59(\mathrm{~d}, J=8.5 \mathrm{~Hz}, 1 \mathrm{H}), 7.50-7.43(\mathrm{~m}, 2 \mathrm{H}), 7.27(\mathrm{~s}$, 4H), 7.09 (s, 1H), 4.34 (q, $J=7.1 \mathrm{~Hz}, 2 \mathrm{H}), 4.19(\mathrm{q}, J=7.1 \mathrm{~Hz}, 2 \mathrm{H}), 2.42(\mathrm{~s}, 3 \mathrm{H}), 1.29$ (d, $J=7.1$ $\mathrm{Hz}, 3 \mathrm{H}), 1.18(\mathrm{~d}, J=7.1 \mathrm{~Hz}, 3 \mathrm{H}) .{ }^{13} \mathrm{C} \mathrm{NMR}\left(75 \mathrm{MHz}, \mathrm{CDCl}_{3}\right) \delta 166.2,159.8,138.3,136.9$, $133.4,132.3,130.7,129.3,127.9,127.8,127.5,126.2,126.1,126.0,125.8,125.6,124.4,121.8$, 61.2, 60.7, 21.1, 14.0, 13.8. Anal. Calcd for $\mathrm{C}_{27} \mathrm{H}_{25} \mathrm{NO}_{4} \mathrm{C}, 75.86 ; \mathrm{H}, 5.89 ; \mathrm{N}, 3.28 \%$. Found: $\mathrm{C}$, 75.82; H, 5.86; N, $3.31 \%$.

Diethyl 1-(4-chlorophenyl)-4-(naphthalen-2-yl)-1H-pyrrole-2,3-dicarboxylate (3n)

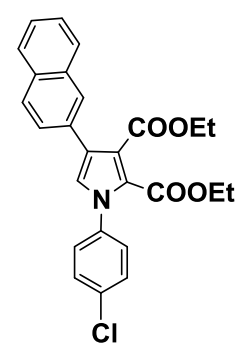


White solid; mp $118-121{ }^{\circ} \mathrm{C}$; IR (neat): 1724, 1707, 1626, $1516 \mathrm{~cm}^{-1} .{ }^{1} \mathrm{H}$ NMR $(300 \mathrm{MHz}$, $\left.\mathrm{CDCl}_{3}\right) \delta 7.91(\mathrm{~s}, 1 \mathrm{H}), 7.86-7.80(\mathrm{~m}, 4 \mathrm{H}), 7.60-7.56(\mathrm{~m}, 1 \mathrm{H}), 7.49-7.47(\mathrm{~m}, 1 \mathrm{H}), 7.46-7.42$ (m, 2H), $7.35-7.33(\mathrm{~m}, 1 \mathrm{H}), 7.32-7.30(\mathrm{~m}, 1 \mathrm{H}), 7.08(\mathrm{~s}, 1 \mathrm{H}), 4.34(\mathrm{q}, J=7.1 \mathrm{~Hz}, 2 \mathrm{H}), 4.20(\mathrm{q}, J$ $=7.1 \mathrm{~Hz}, 2 \mathrm{H}), 1.29(\mathrm{t}, J=7.1 \mathrm{~Hz}, 3 \mathrm{H}), 1.21(\mathrm{t}, J=7.1 \mathrm{~Hz}, 3 \mathrm{H}) \cdot{ }^{13} \mathrm{C} \mathrm{NMR}\left(75 \mathrm{MHz}, \mathrm{CDCl}_{3}\right) \delta$ $166.1,159.7,138.1,134.5,133.5,132.6,130.5,129.1,128.1,127.9,127.6{ }^{*} 126.25,126.22$, 126.20, 125.90, 125.86, 125.0, 123.4, 122.8, 61.4, 61.0, 14.1, 13.9. Anal. Calcd for $\mathrm{C}_{26} \mathrm{H}_{22} \mathrm{ClNO}_{4}$ : C, 69.72; H, 4.95; N, $3.13 \%$. Found: C, 69.65; H, 4.90; N, $3.17 \%$. *(Two carbon signals merged here)

\section{Diethyl 1-(4-bromophenyl)-4-(naphthalen-1-yl)-1H-pyrrole-2,3-dicarboxylate (3o)}

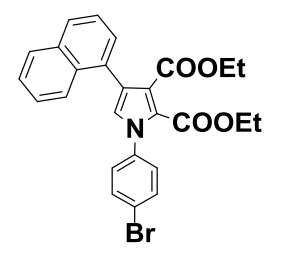

White viscous solid; IR (neat): 1726, 1701, 1593, $1442 \mathrm{~cm}^{-1} .{ }^{1} \mathrm{H}$ NMR $\left(300 \mathrm{MHz}, \mathrm{CDCl}_{3}\right) \delta 7.96-$ $7.93(\mathrm{~m}, 1 \mathrm{H}), 7.89-7.83(\mathrm{~m}, 2 \mathrm{H}), 7.61(\mathrm{~d}, J=8.7 \mathrm{~Hz}, 2 \mathrm{H}), 7.48-7.44(\mathrm{~m}, 4 \mathrm{H}), 7.31(\mathrm{~d}, J=8.7$ $\mathrm{Hz}, 2 \mathrm{H}), 6.95(\mathrm{~s}, 1 \mathrm{H}), 4.23(\mathrm{q}, J=7.1 \mathrm{~Hz}, 2 \mathrm{H}), 3.97(\mathrm{q}, J=7.1 \mathrm{~Hz}, 2 \mathrm{H}), 1.22(\mathrm{~d}, J=7.1 \mathrm{~Hz}, 3 \mathrm{H})$, $0.82(\mathrm{~d}, J=7.1 \mathrm{~Hz}, 3 \mathrm{H}) .{ }^{13} \mathrm{C} \mathrm{NMR}\left(75 \mathrm{MHz}, \mathrm{CDCl}_{3}\right) \delta 164.8,160.3,138.3,133.5,132.6,132.1$, $131.1,128.1,127.9,127.7,127.5,126.2,125.9,125.7{ }^{*} 125.1,124.3,123.8,123.1,122.3,61.3$, 60.7, 13.9, 13.5. Anal. Calcd for : $\mathrm{C}_{26} \mathrm{H}_{22} \mathrm{BrNO}_{4} \mathrm{C}, 63.43 ; \mathrm{H}, 4.50 ; \mathrm{N}, 2.84 \%$. Found: C, 63.37; H, 4.46; N, $2.87 \%$ (*Two carbon signals merged here).

Diethyl 1-(naphthalen-1-yl)-4-(naphthalen-2-yl)-1H-pyrrole-2,3-dicarboxylate (3p) 


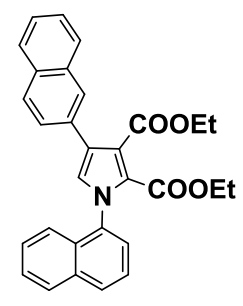

Pale brown solid; mp 216-218 ${ }^{\circ} \mathrm{C}$; IR (neat): 1731, 1706, 1625, $1514 \mathrm{~cm}^{-1} .{ }^{1} \mathrm{H}$ NMR $(300 \mathrm{MHz}$, $\left.\mathrm{CDCl}_{3}\right) \delta 7.98-7.92(\mathrm{~m}, 3 \mathrm{H}), 7.87-7.81(\mathrm{~m}, 3 \mathrm{H}), 7.63(\mathrm{dd}, J=8.5,1.7 \mathrm{~Hz}, 1 \mathrm{H}), 7.56-7.45(\mathrm{~m}$, 7H), $7.19(\mathrm{~s}, 1 \mathrm{H}), 4.40(\mathrm{q}, J=7.1 \mathrm{~Hz}, 2 \mathrm{H}), 3.97(\mathrm{q}, J=6 \mathrm{~Hz}, 1 \mathrm{H}), 3.98(\mathrm{q}, J=6 \mathrm{~Hz}, 1 \mathrm{H}), 1.33(\mathrm{t}, J$ $=7.1 \mathrm{~Hz}, 3 \mathrm{H}), 0.87(\mathrm{t}, J=7.1 \mathrm{~Hz}, 3 \mathrm{H}) .{ }^{13} \mathrm{C} \mathrm{NMR}\left(75 \mathrm{MHz}, \mathrm{CDCl}_{3}\right) \delta 166.5,159.3,136.6,133.7$, 133.5, 132.4, 130.8, 130.7, 129.2, 128.1, 128.0, 127.9, 127.6, 127.4, 126.9, 126.7, 126.2, 126.1, 126.0, 125.7, 124.9, 124.6, 124.5, 124.5, 122.5, 122.3, 61.5, 60.5, 14.1, 13.5. Anal. Calcd for $\mathrm{C}_{30} \mathrm{H}_{25} \mathrm{NO}_{4}$ : C, 77.74; H, 5.44; N, 3.02\%. Found: C, 77.79; H, 5.41; N, $3.08 \%$. ESI-MS m/z calcd 463.18, found 463.39.

\section{Diethyl 1-(4-methoxyphenyl)-4-(naphthalen-2-yl)-1H-pyrrole-2,3-dicarboxylate (3q)}

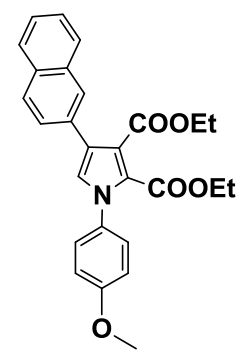

White solid; mp $85-88^{\circ} \mathrm{C}$; IR (neat): 1708, 1628, 1598, $1458 \mathrm{~cm}^{-1} .{ }^{1} \mathrm{H}$ NMR $\left(300 \mathrm{MHz}, \mathrm{CDCl}_{3}\right)$ $\delta 7.92(\mathrm{~s}, 1 \mathrm{H}), 7.83(\mathrm{t}, J=7.2 \mathrm{~Hz}, 3 \mathrm{H}), 7.59(\mathrm{dd}, J=8.5,1.6 \mathrm{~Hz}, 1 \mathrm{H}), 7.49-7.45(\mathrm{~m}, 2 \mathrm{H}), 7.31$ $(\mathrm{d}, J=8.8 \mathrm{~Hz}, 2 \mathrm{H}), 7.07(\mathrm{~s}, 1 \mathrm{H}), 6.97(\mathrm{~d}, J=8.9 \mathrm{~Hz}, 2 \mathrm{H}), 4.34(\mathrm{q}, J=7.1 \mathrm{~Hz}, 2 \mathrm{H}), 4.19(\mathrm{q}, J=$ $7.1 \mathrm{~Hz}, 2 \mathrm{H}), 3.86(\mathrm{~s}, 3 \mathrm{H}), 1.29(\mathrm{t}, J=7.2 \mathrm{~Hz}, 3 \mathrm{H}), 1.20(\mathrm{t}, J=7.1 \mathrm{~Hz}, 3 \mathrm{H}) .{ }^{13} \mathrm{C}$ NMR $(75 \mathrm{MHz}$, $\left.\mathrm{CDCl}_{3}\right) \delta 166.2,159.7,159.3,133.3,132.2,132.2,130.6,127.8,127.7,127.4,127.2,126.1,126.1$, 
126.0, 125.9, 125.6, 124.1, 123.4, 121.7, 113.7, 61.1, 60.6, 55.3, 13.9, 13.8. Anal. Calcd for $\mathrm{C}_{27} \mathrm{H}_{25} \mathrm{NO}_{5}: \mathrm{C}, 73.12 ; \mathrm{H}, 5.68 ; \mathrm{N}, 3.16 \%$. Found: C, 73.07; H, 5.71; N, $3.18 \%$. ESI-MS m/z calcd $[\mathrm{M}+\mathrm{H}]^{+}$444.17, found 444.07.

Dimethyl 1-(4-chlorophenyl)-4-phenyl-1H-pyrrole-2,3-dicarboxylate $(3 \mathbf{r})^{1}$

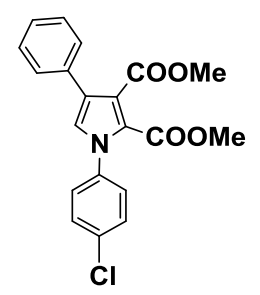

Yellow solid; ${ }^{1} \mathrm{H}$ NMR $\left(300 \mathrm{MHz}, \mathrm{CDCl}_{3}\right) \delta 7.44-7.41(\mathrm{~m}, 4 \mathrm{H}), 7.40-7.35(\mathrm{~m}, 2 \mathrm{H}), 7.32-7.25$ (m, 3H), 6.97 (s, 1H), 3.85 (s, 3H), 3.72 (s, 3H). ${ }^{13} \mathrm{C}$ NMR $\left(75 \mathrm{MHz}, \mathrm{CDCl}_{3}\right) \delta 166.5,160.0,137.8$, $134.3,132.7,129.0,128.5,127.4,127.4,127.2,125.8,124.9,122.7,122.2,52.4,51.9$.

Dimethyl 1-(4-bromophenyl)-4-phenyl-1H-pyrrole-2,3-dicarboxylate (3s)

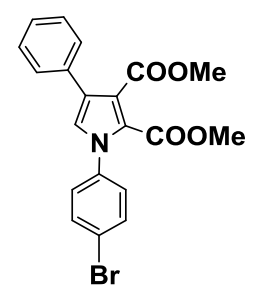

Colourless viscous liquid; IR (neat):1707, 1600, $1436 \mathrm{~cm}^{-1} .{ }^{1} \mathrm{H}$ NMR $\left(300 \mathrm{MHz}, \mathrm{CDCl}_{3}\right) \delta 7.59$ $(\mathrm{d}, J=8.7 \mathrm{~Hz}, 2 \mathrm{H}), 7.45-7.40(\mathrm{~m}, 3 \mathrm{H}), 7.37-7.30(\mathrm{~m}, 2 \mathrm{H}), 7.24(\mathrm{~d}, J=8.7 \mathrm{~Hz}, 2 \mathrm{H}), 6.98(\mathrm{~s}$, 1H), 3.85 (s, 3H), $3.73(\mathrm{~s}, 3 \mathrm{H}) .{ }^{13} \mathrm{C} \mathrm{NMR}\left(75 \mathrm{MHz}, \mathrm{CDCl}_{3}\right) \delta 166.5,160.2,138.5,132.9,132.1$, 128.6, 127.8, 127.7, 127.3, 125.8, 125.2, 123.0, 122.5, 122.4, 52.3, 51.9. Anal. Calcd for $\mathrm{C}_{20} \mathrm{H}_{16} \mathrm{BrNO}_{4}$ : C, 57.99; H, 3.89; N, $3.38 \%$. Found: C, 57.94; H, 3.92; N, $3.32 \%$.

Dimethyl 1-(4-chlorophenyl)-4-(p-tolyl)-1H-pyrrole-2,3-dicarboxylate (3t) 


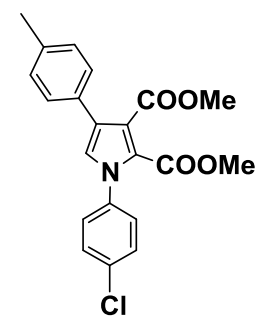

White solid; mp $101-121{ }^{\circ} \mathrm{C}$; IR (neat): 1727, 1699, 1555, $1434 \mathrm{~cm}^{-1} .{ }^{1} \mathrm{H}$ NMR $(300 \mathrm{MHz}$, $\left.\mathrm{CDCl}_{3}\right) \delta 7.42(\mathrm{~d}, J=8.7 \mathrm{~Hz}, 2 \mathrm{H}), 7.34-7.30(\mathrm{~m}, 4 \mathrm{H}), 7.18(\mathrm{~d}, J=8.0 \mathrm{~Hz}, 2 \mathrm{H}), 6.95(\mathrm{~s}, 1 \mathrm{H}), 3.85$ (s, 3H), $3.72(\mathrm{~s}, 3 \mathrm{H}), 2.36(\mathrm{~s}, 3 \mathrm{H}) .{ }^{13} \mathrm{C}$ NMR $\left(75 \mathrm{MHz}, \mathrm{CDCl}_{3}\right) \delta$ 166.6, 160.1, 137.9, 137.0, 134.4, $129.8,129.3,{ }^{*} 129.0,127.5,127.4,125.7,125.0,122.3,52.4,51.9,21.1$. Anal. Calcd for $\mathrm{C}_{21} \mathrm{H}_{18} \mathrm{ClNO}_{4}: \mathrm{C}, 65.71 ; \mathrm{H}, 4.73 ; \mathrm{N}, 3.65 \%$. Found: $\mathrm{C}, 65.66 ; \mathrm{H}, 4.77 ; \mathrm{N}, 3.62 \%$. *(Two carbon signals merged here)

\section{Dimethyl 1,4-bis(4-methoxyphenyl)-1H-pyrrole-2,3-dicarboxylate (3u)}

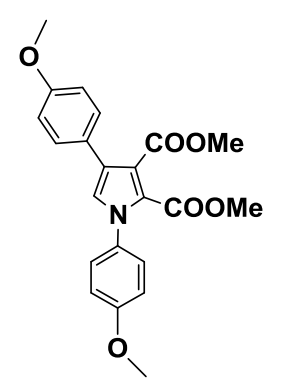

Pale yellow solid; mp $91-93{ }^{\circ} \mathrm{C}$; IR (neat): 1723, 1697, $1497 \mathrm{~cm}^{-1} .{ }^{1} \mathrm{H}$ NMR (300 MHz, $\mathrm{CDCl}_{3}$ ) $\delta 7.37(\mathrm{~d}, J=8.8 \mathrm{~Hz}, 2 \mathrm{H}), 7.28-7.25(\mathrm{~m}, 2 \mathrm{H}), 6.96(\mathrm{~s}, 1 \mathrm{H}), 6.93-6.90(\mathrm{~m}, 4 \mathrm{H}), 3.85(\mathrm{~s}, 3 \mathrm{H})$, $3.84(\mathrm{~s}, 3 \mathrm{H}), 3.82(\mathrm{~s}, 3 \mathrm{H}), 3.71(\mathrm{~s}, 3 \mathrm{H}) .{ }^{13} \mathrm{C} \mathrm{NMR}\left(75 \mathrm{MHz}, \mathrm{CDCl}_{3}\right) \delta 166.7,160.5,159.5,158.9$, 132.4, 128.9, 127.3, 125.8, 125.7, 124.4, 123.3, 121.3, 114.0, 113.7, 55.5, 55.3, 52.2, 51.8. Anal. Calcd for $\mathrm{C}_{22} \mathrm{H}_{21} \mathrm{NO}_{6}$ : C, 66.83; H, 5.35; N, $3.54 \%$. Found: C, 66.87; H, 5.31; N, $3.57 \%$. 


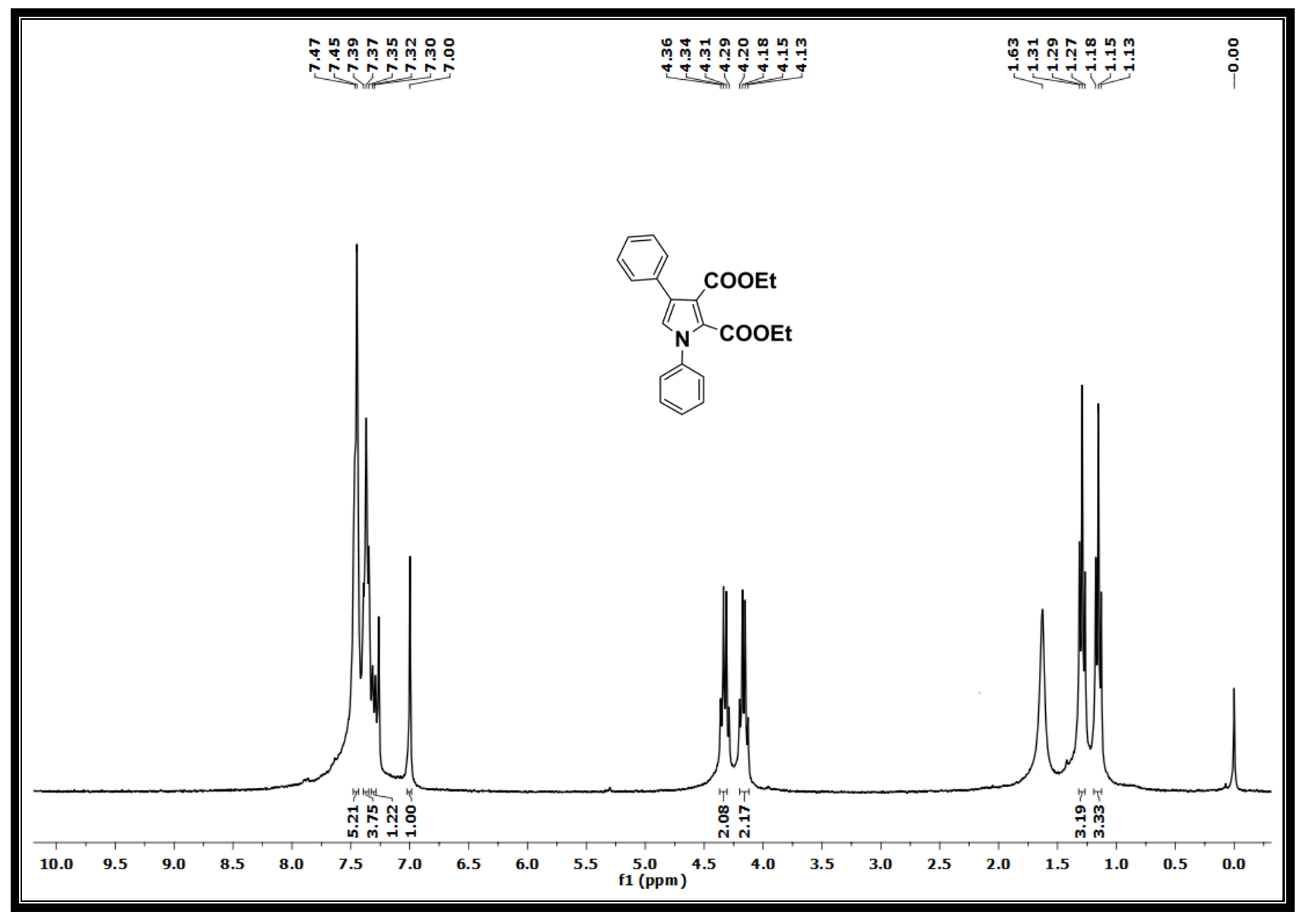

Fig. 1. ${ }^{1} \mathrm{H}$ NMR spectrum of $\mathbf{3 a}$ 


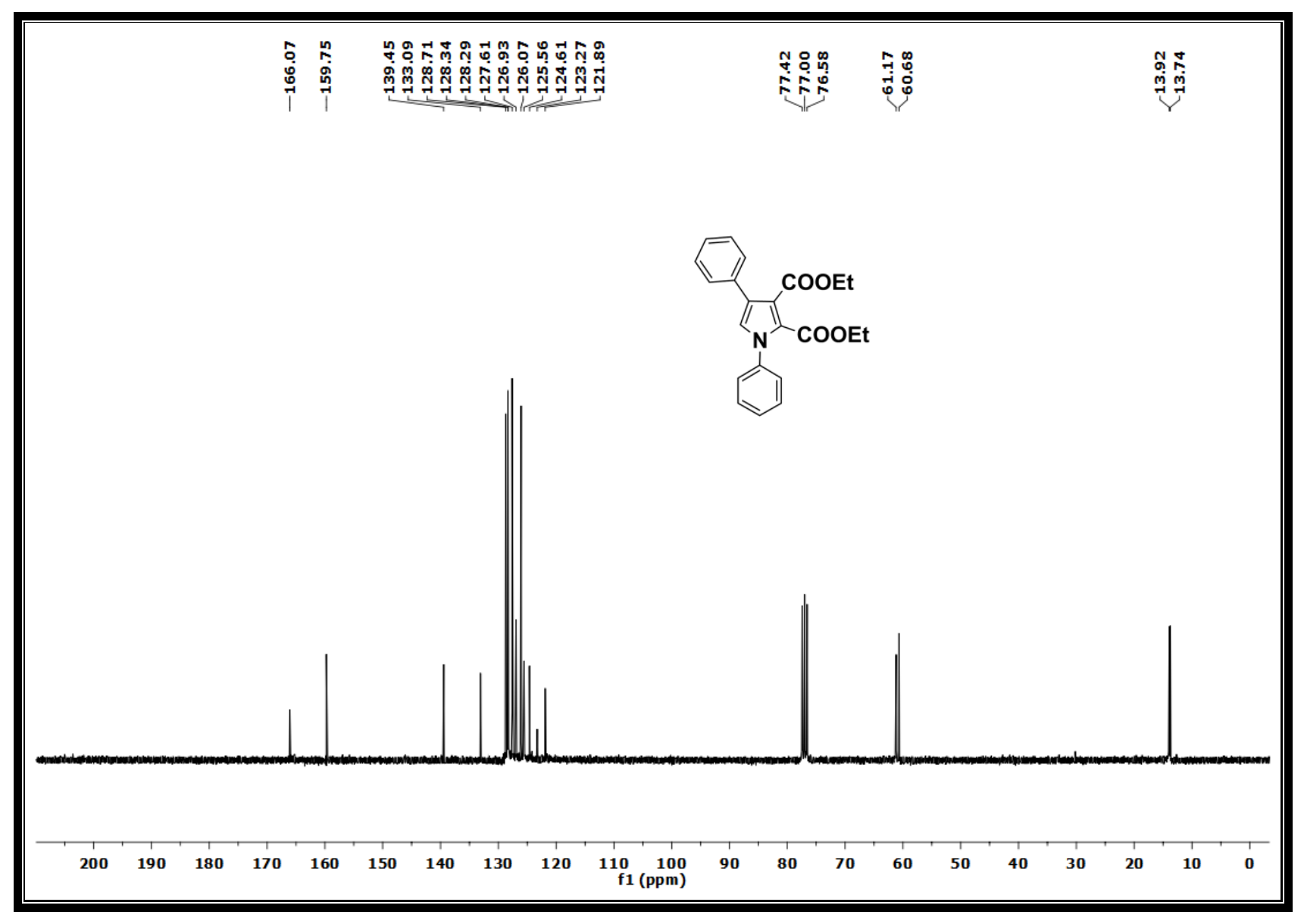

Fig.2. ${ }^{13} \mathrm{C}$ NMR spectrum 3a 


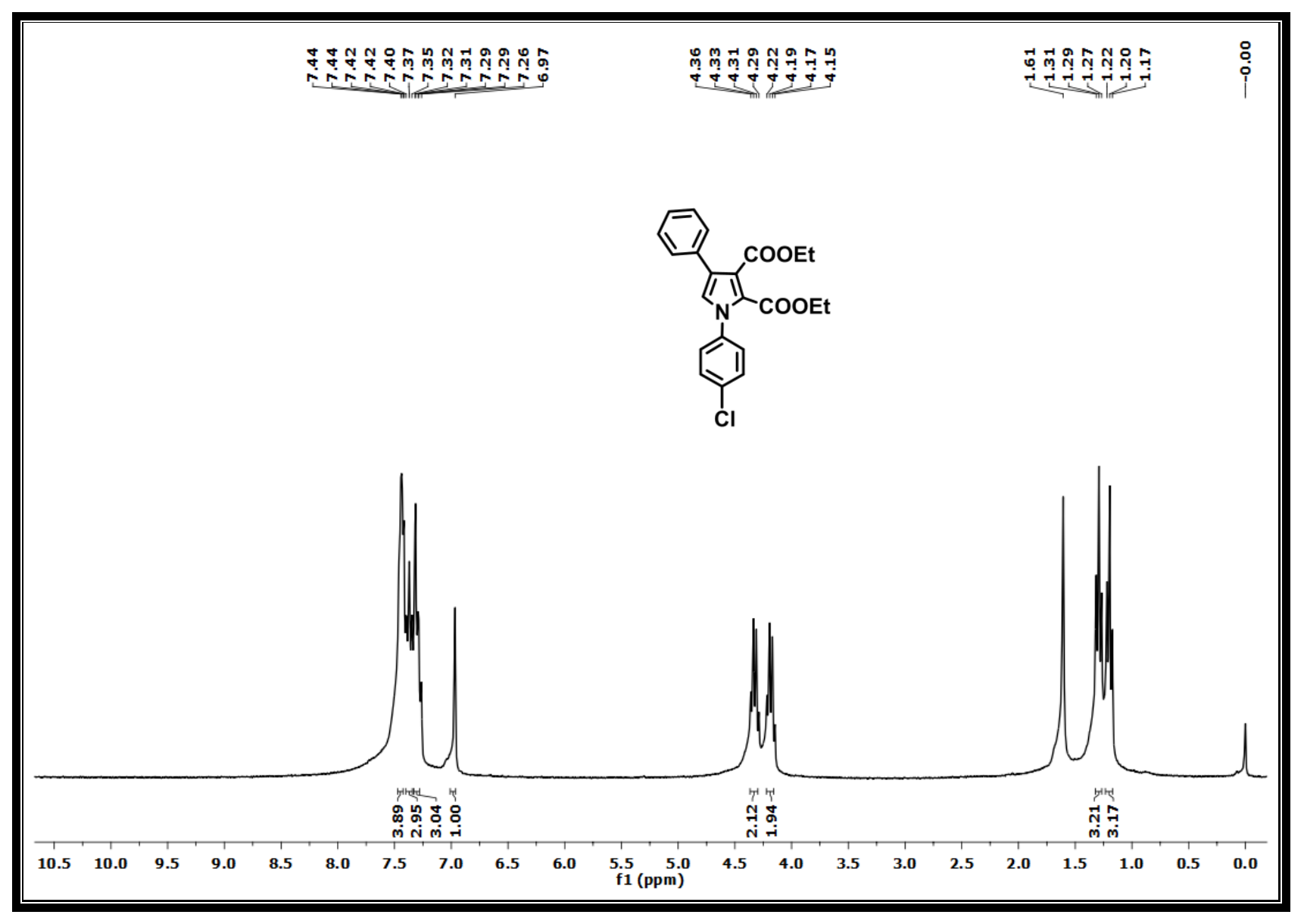

Fig. 3. ${ }^{1} \mathrm{H}$ NMR spectrum of $\mathbf{3 b}$ 


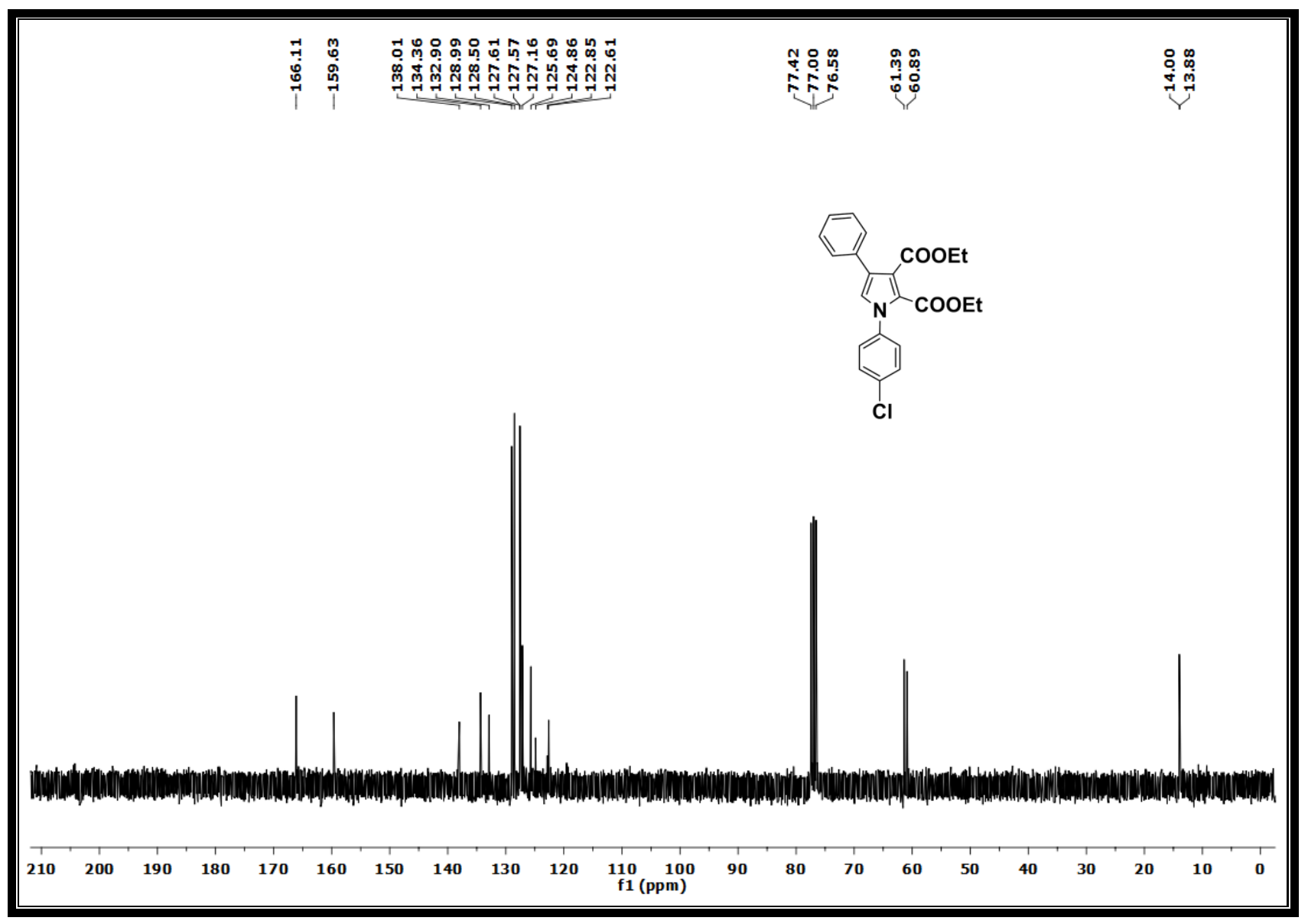

Fig.4. ${ }^{13} \mathrm{C}$ NMR spectrum $\mathbf{3 b}$ 


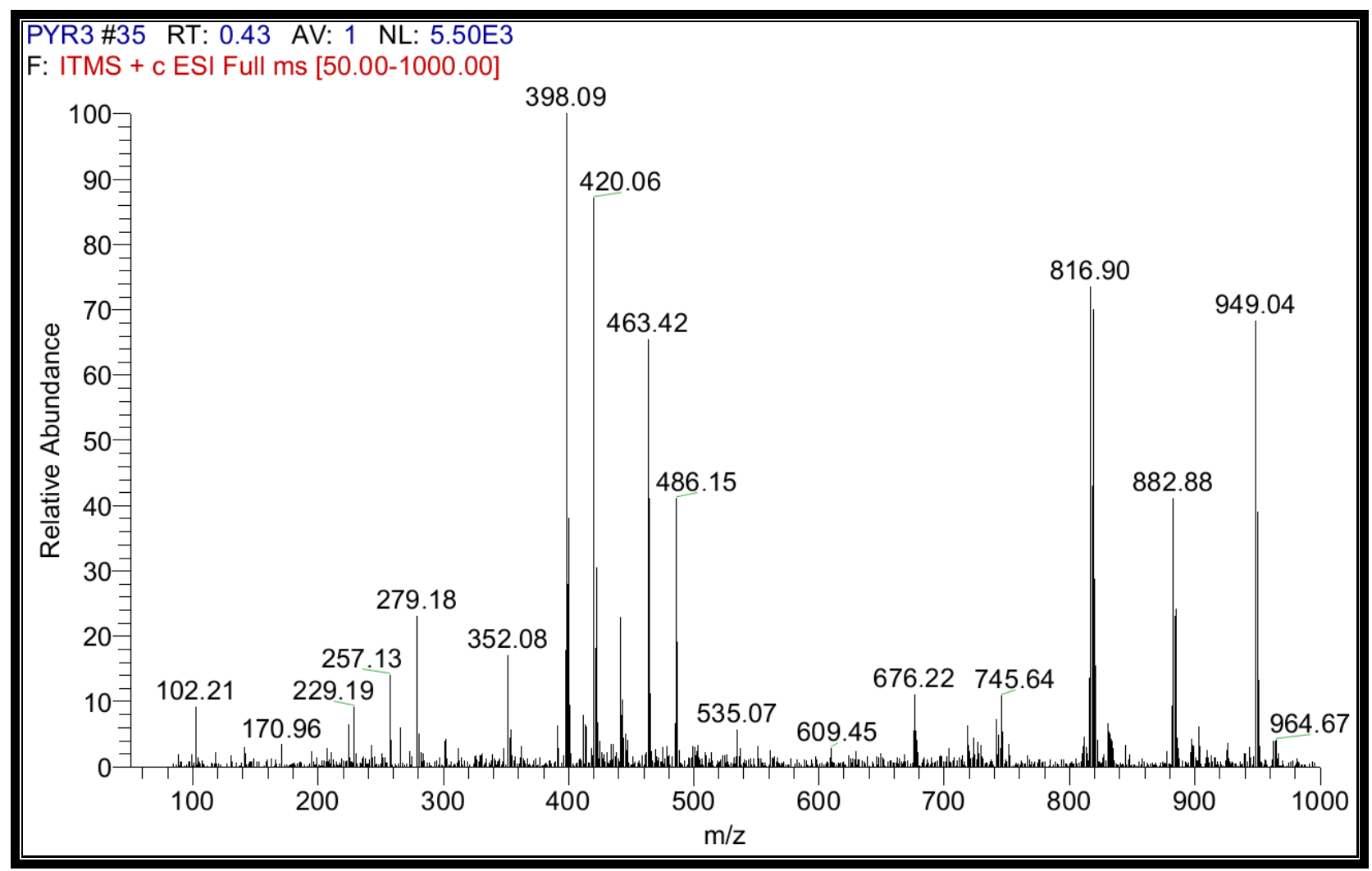

Fig.5. Mass spectrum of $\mathbf{3 b}$ 


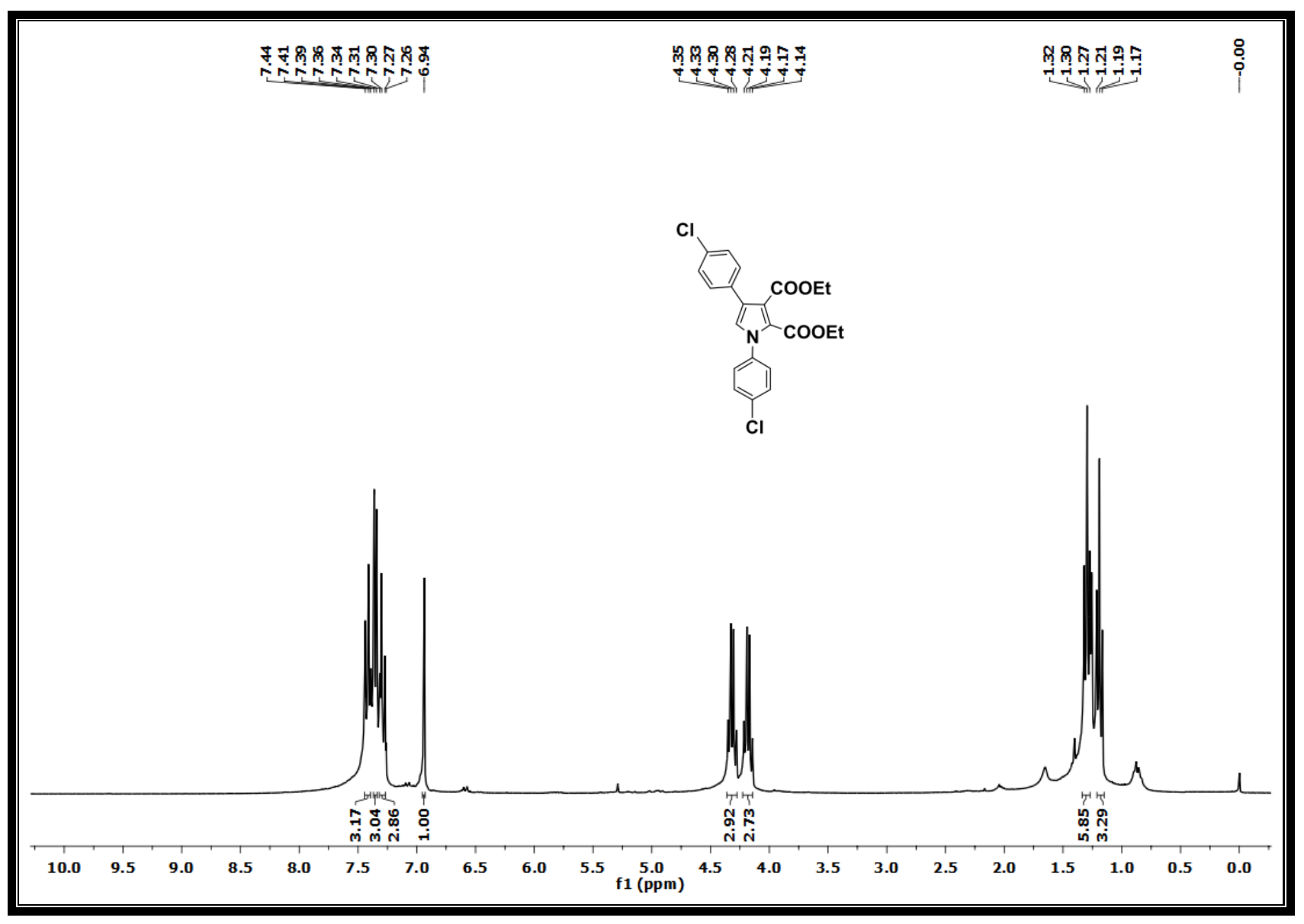

Fig.6. ${ }^{1} \mathrm{H}$ NMR spectrum of $\mathbf{3 c}$ 


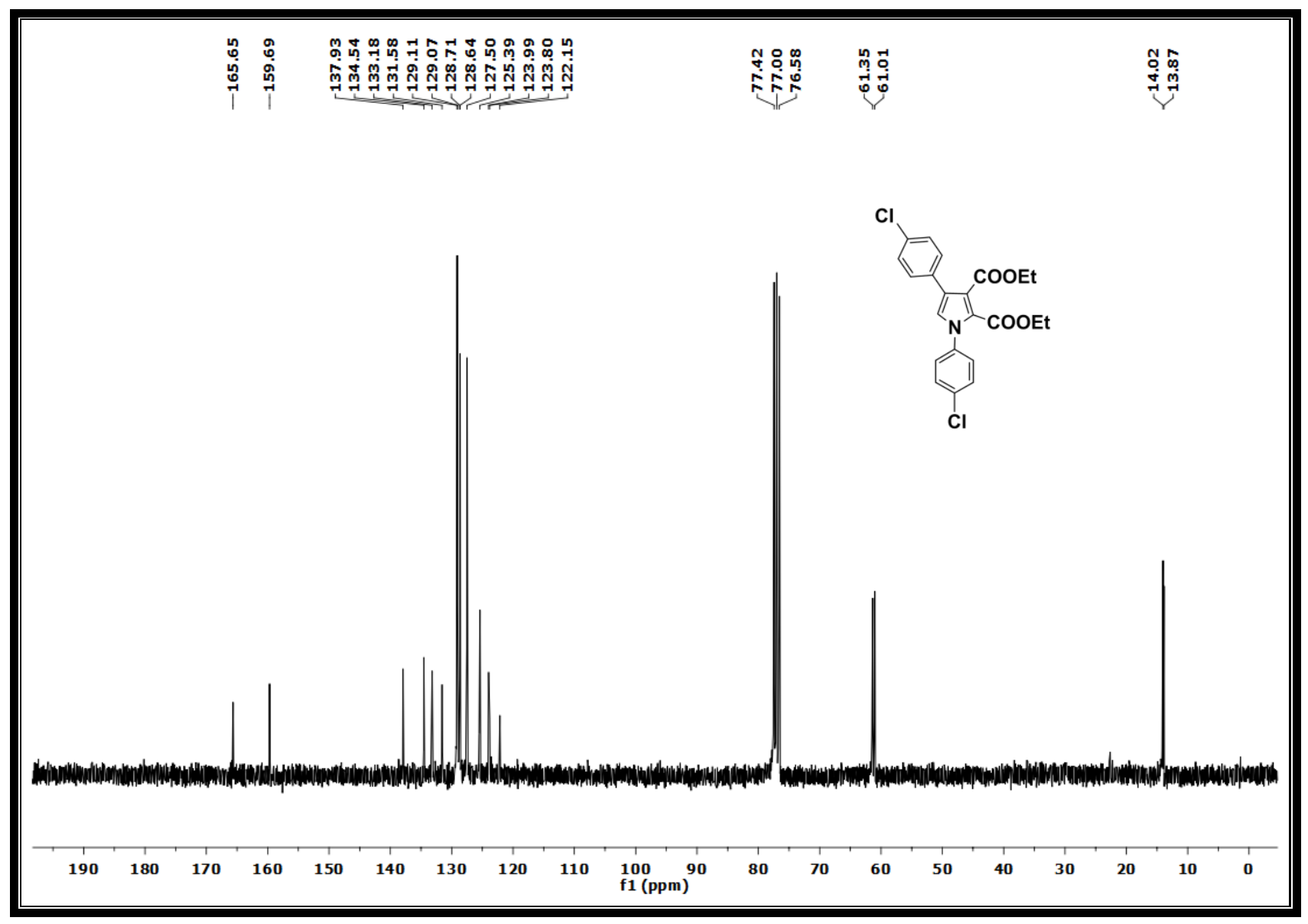

Fig.7. ${ }^{13} \mathrm{C}$ NMR spectrum 3c 


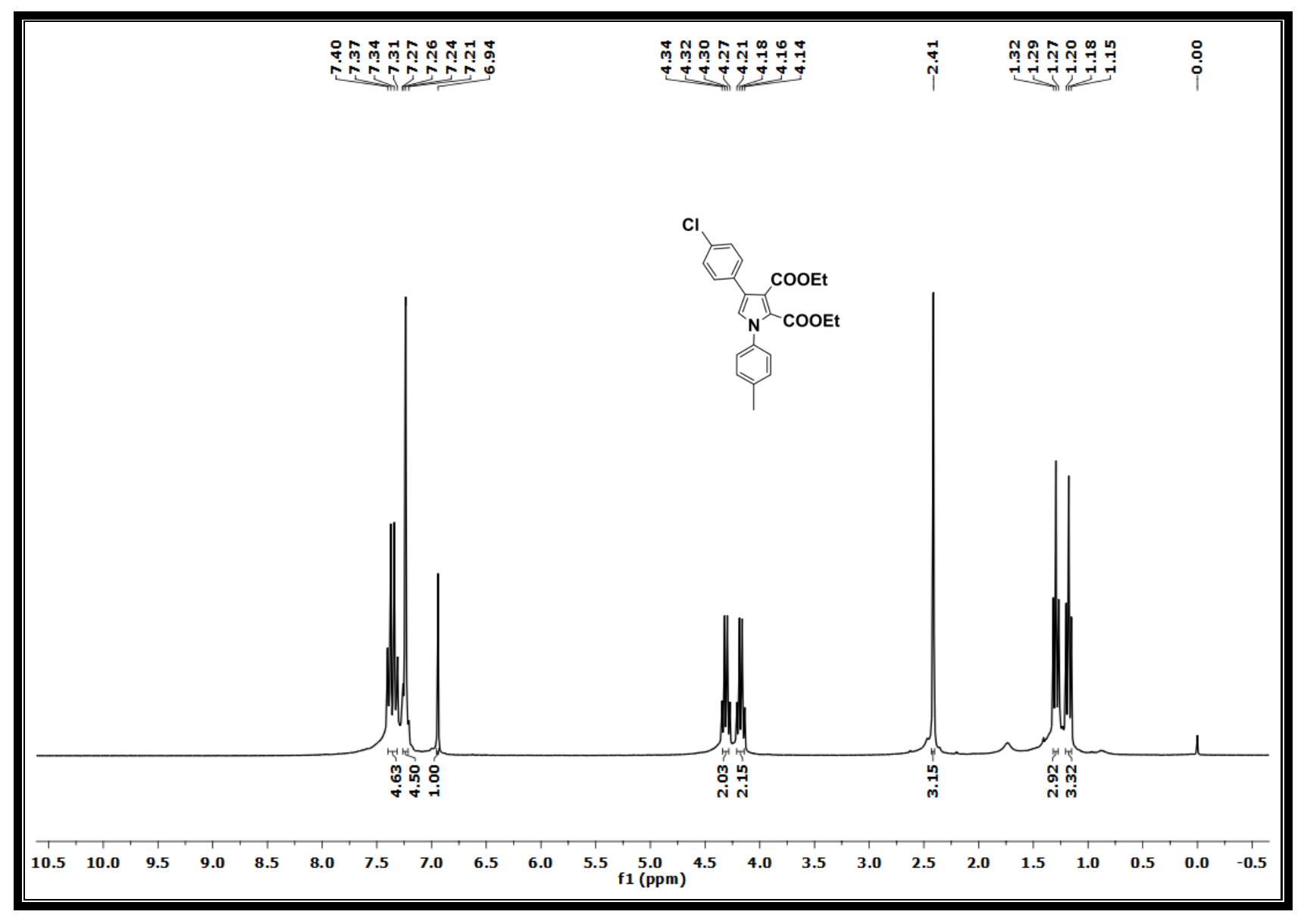

Fig.8. ${ }^{1} \mathrm{H}$ NMR spectrum of $\mathbf{3 d}$ 


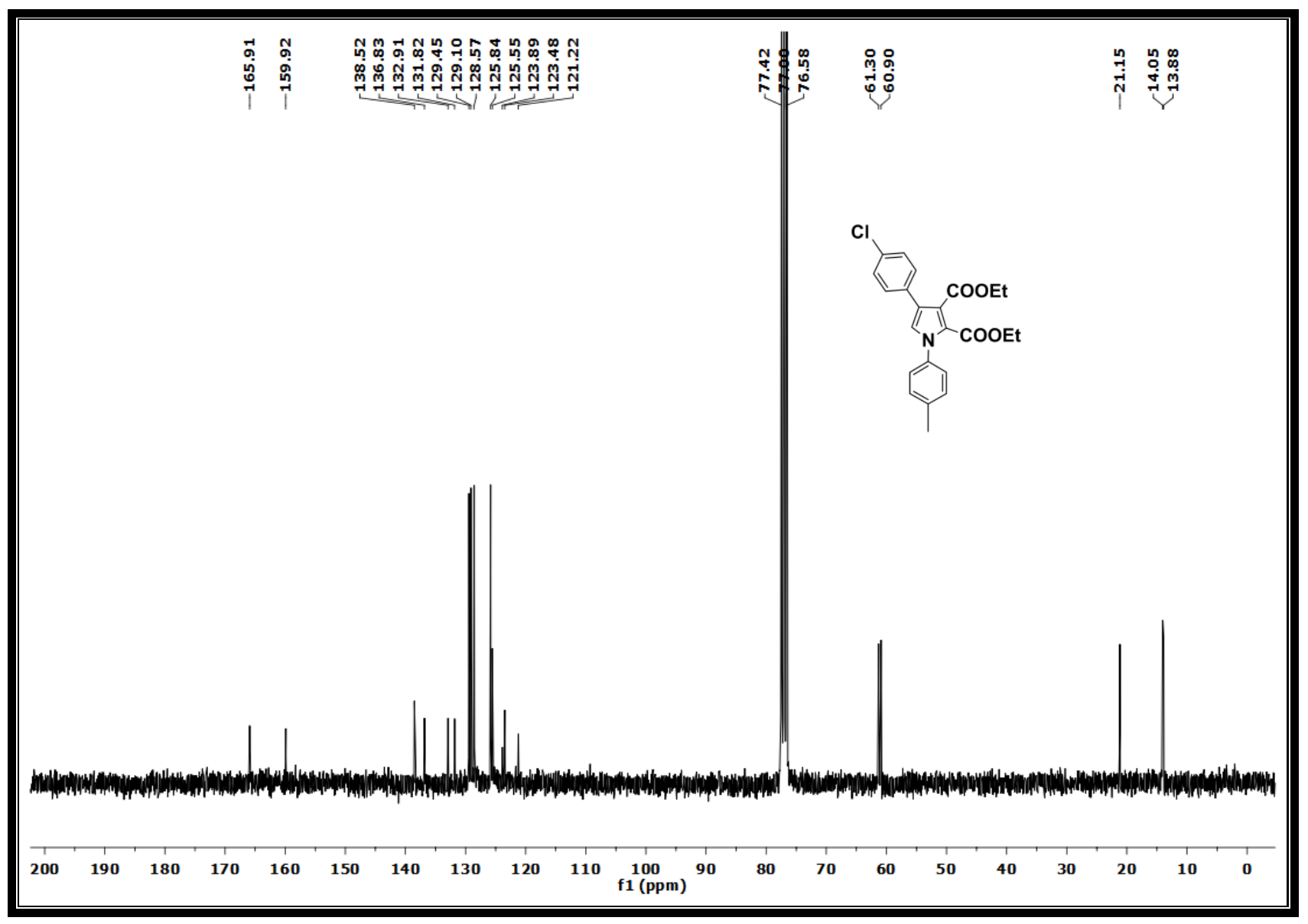

Fig.9. ${ }^{13} \mathrm{C}$ NMR spectrum 3d 


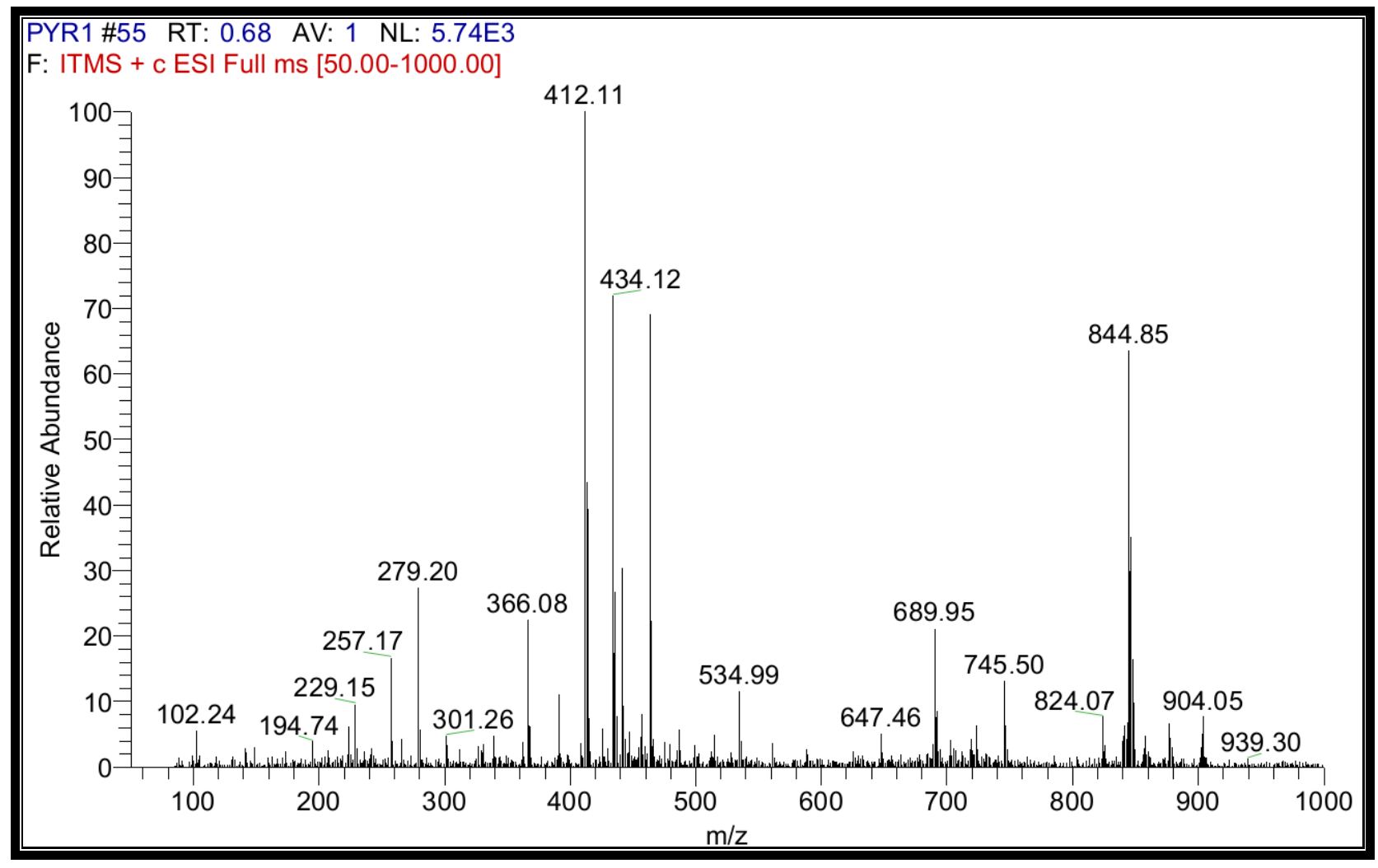

Fig.10. Mass spectrum of $\mathbf{3 d}$ 


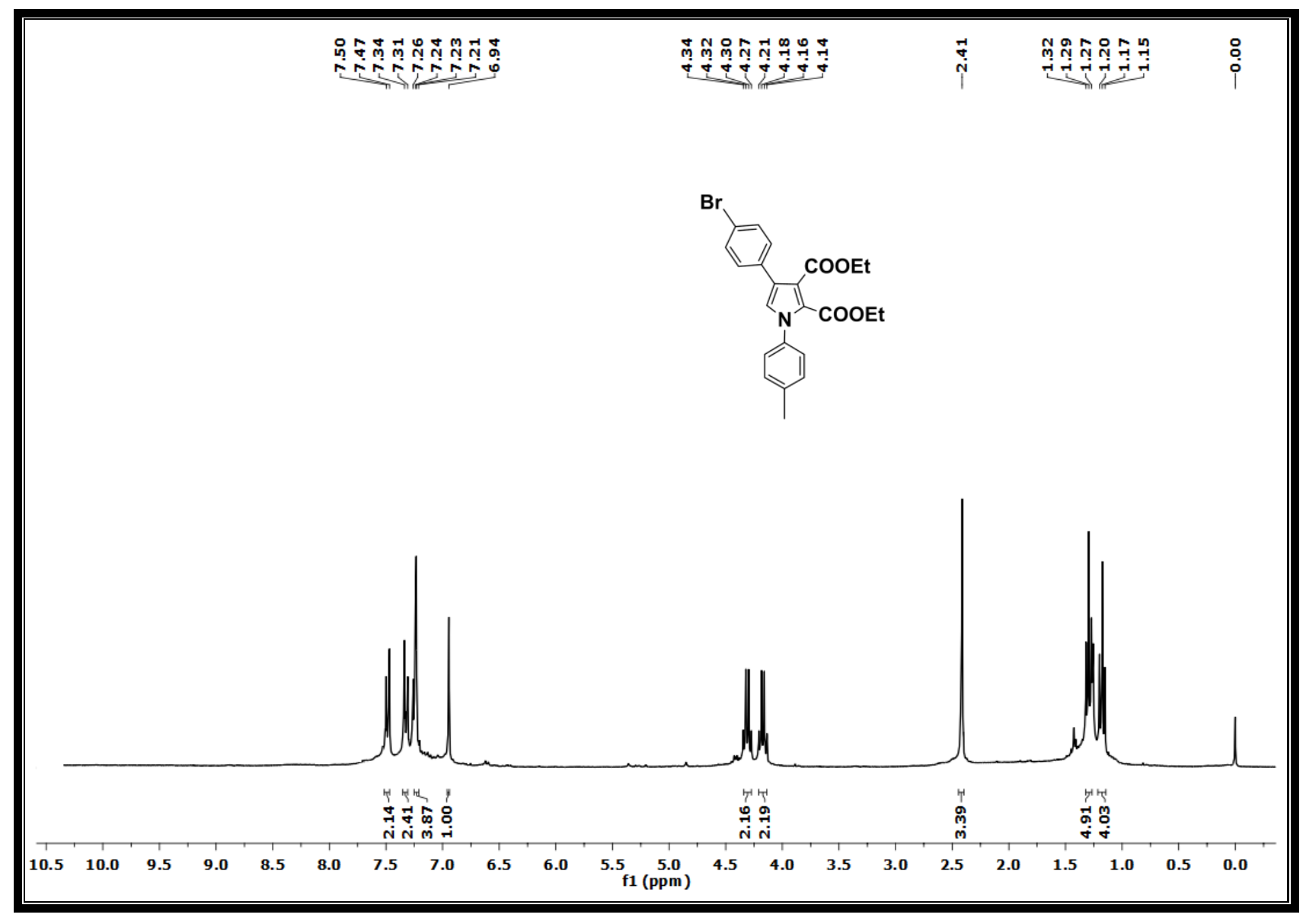

Fig.11. ${ }^{1} \mathrm{H}$ NMR spectrum of $\mathbf{3 e}$ 


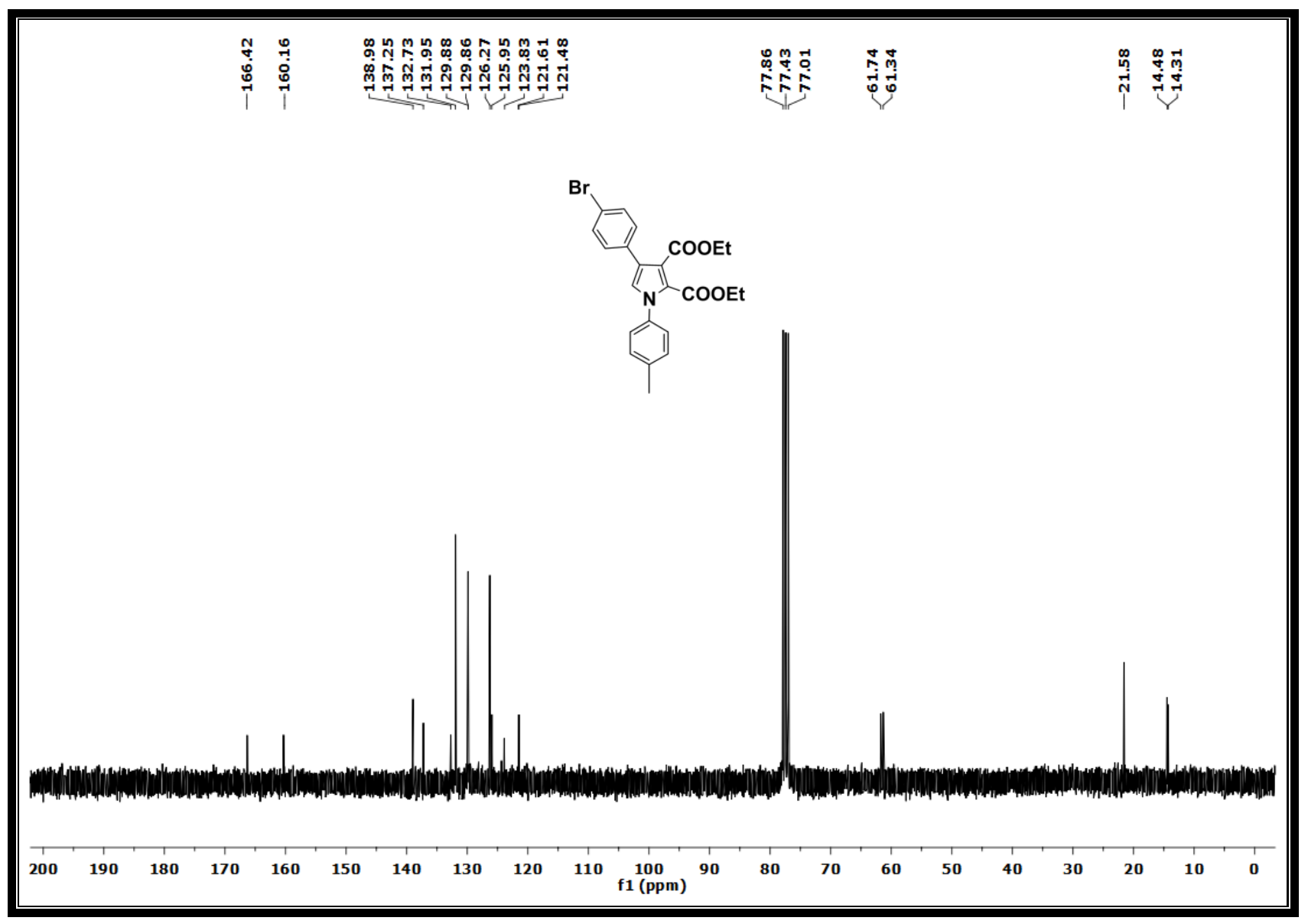

Fig. 12. ${ }^{13} \mathrm{C}$ NMR spectrum $3 \mathbf{e}$ 


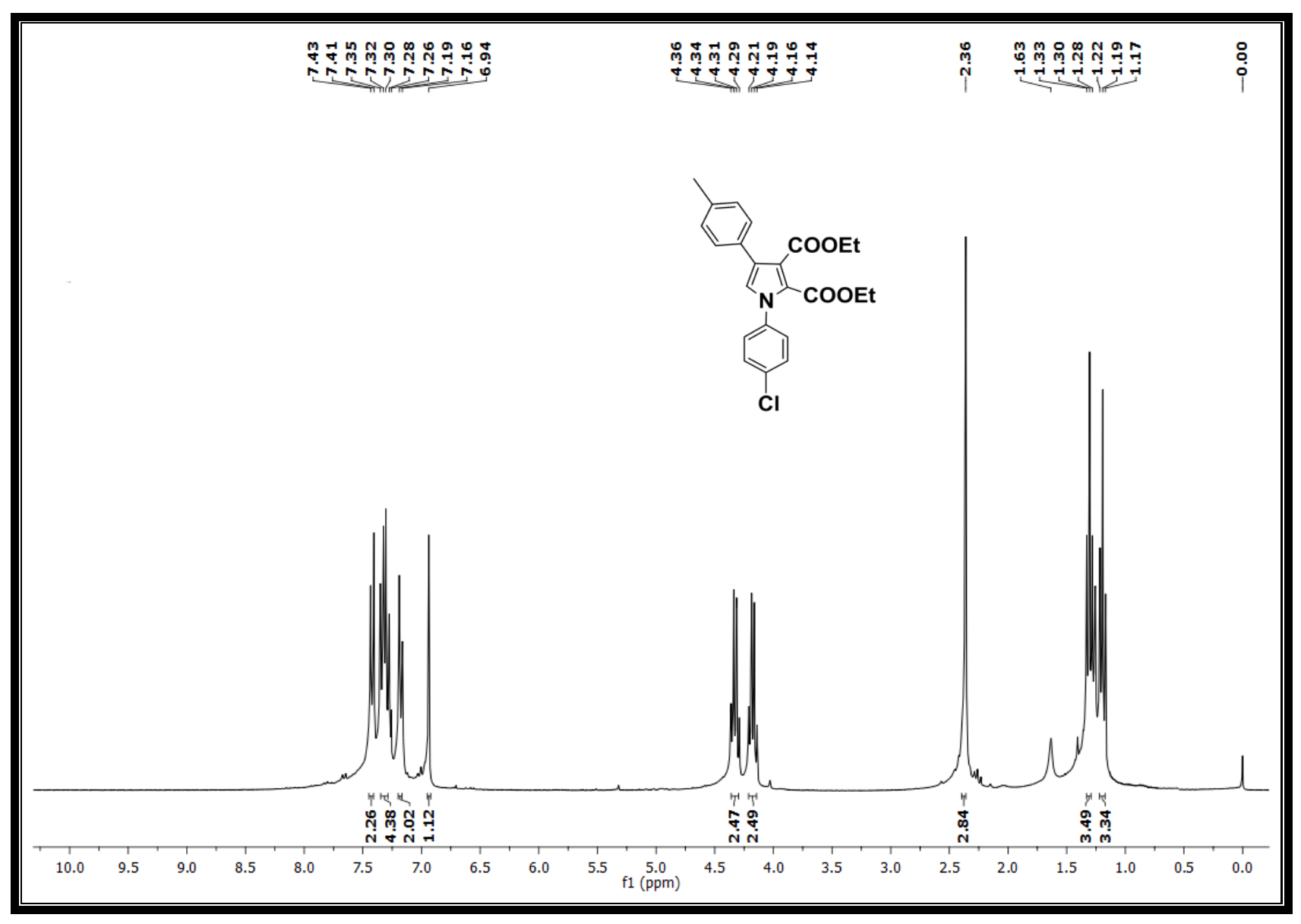

Fig. 13. ${ }^{1} \mathrm{H}$ NMR spectrum of $\mathbf{3 f}$ 


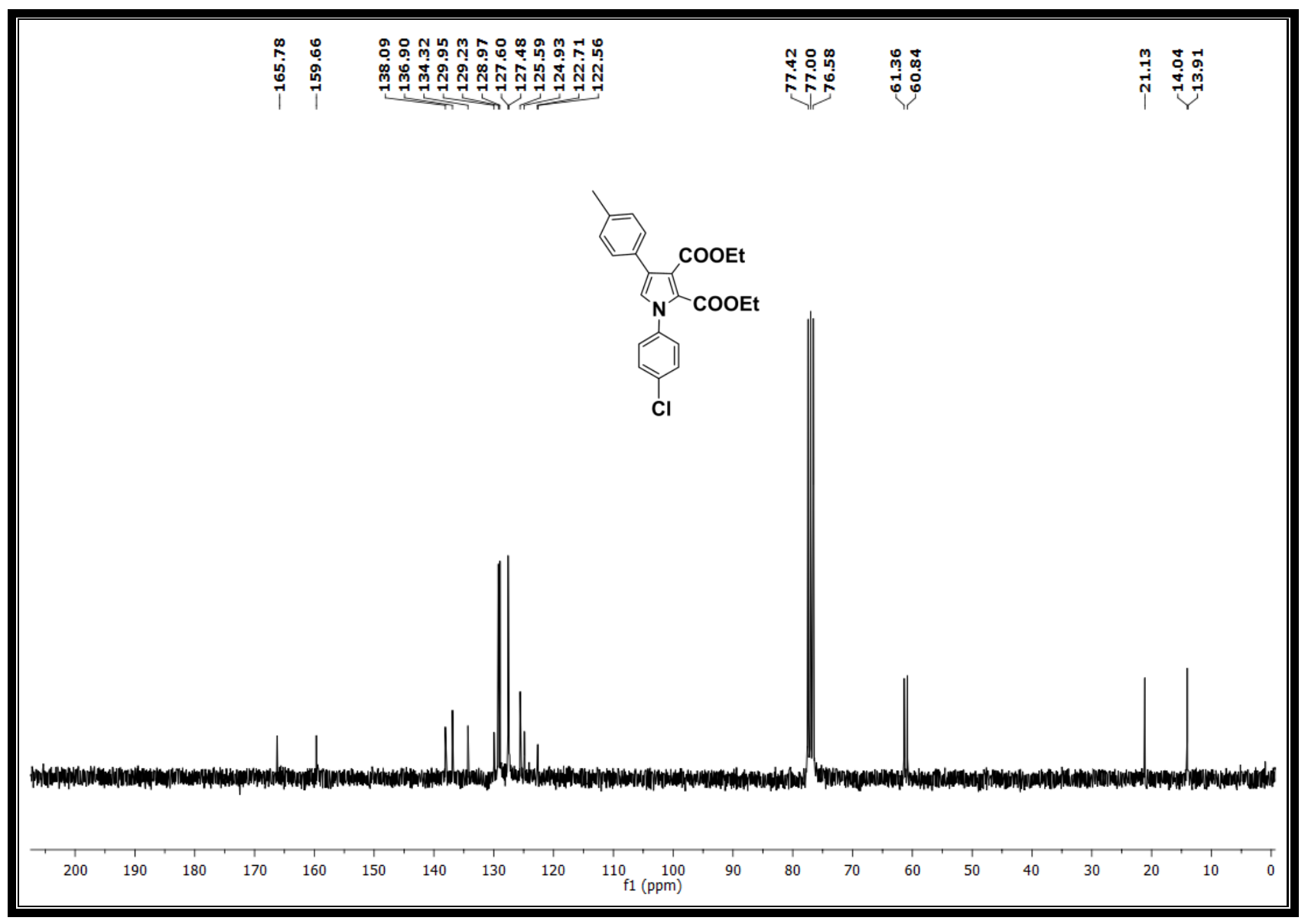

Fig. 14. ${ }^{13} \mathrm{C}$ NMR spectrum $\mathbf{3 f}$ 


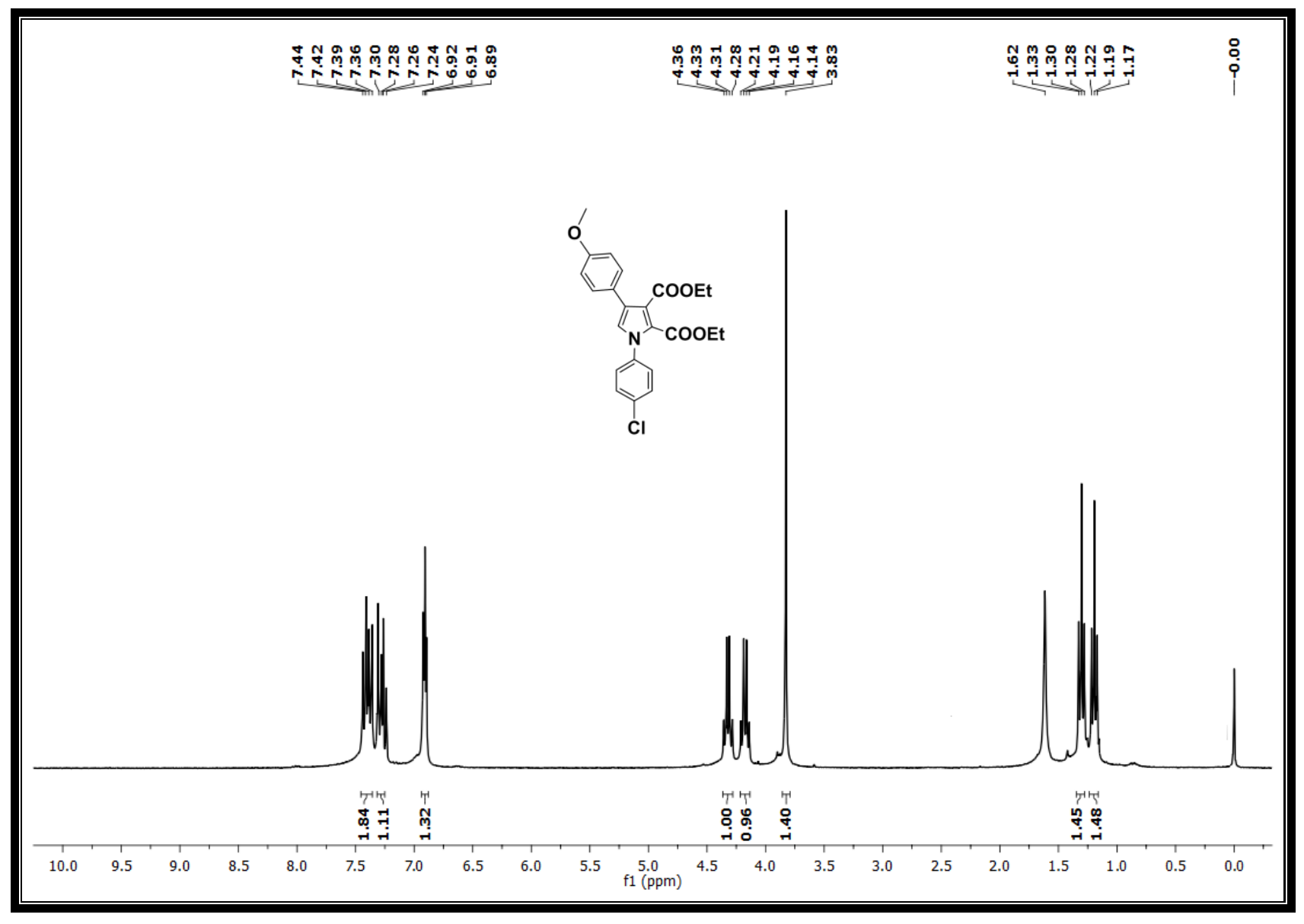

Fig. 15. ${ }^{1} \mathrm{H}$ NMR spectrum of $\mathbf{3 g}$ 


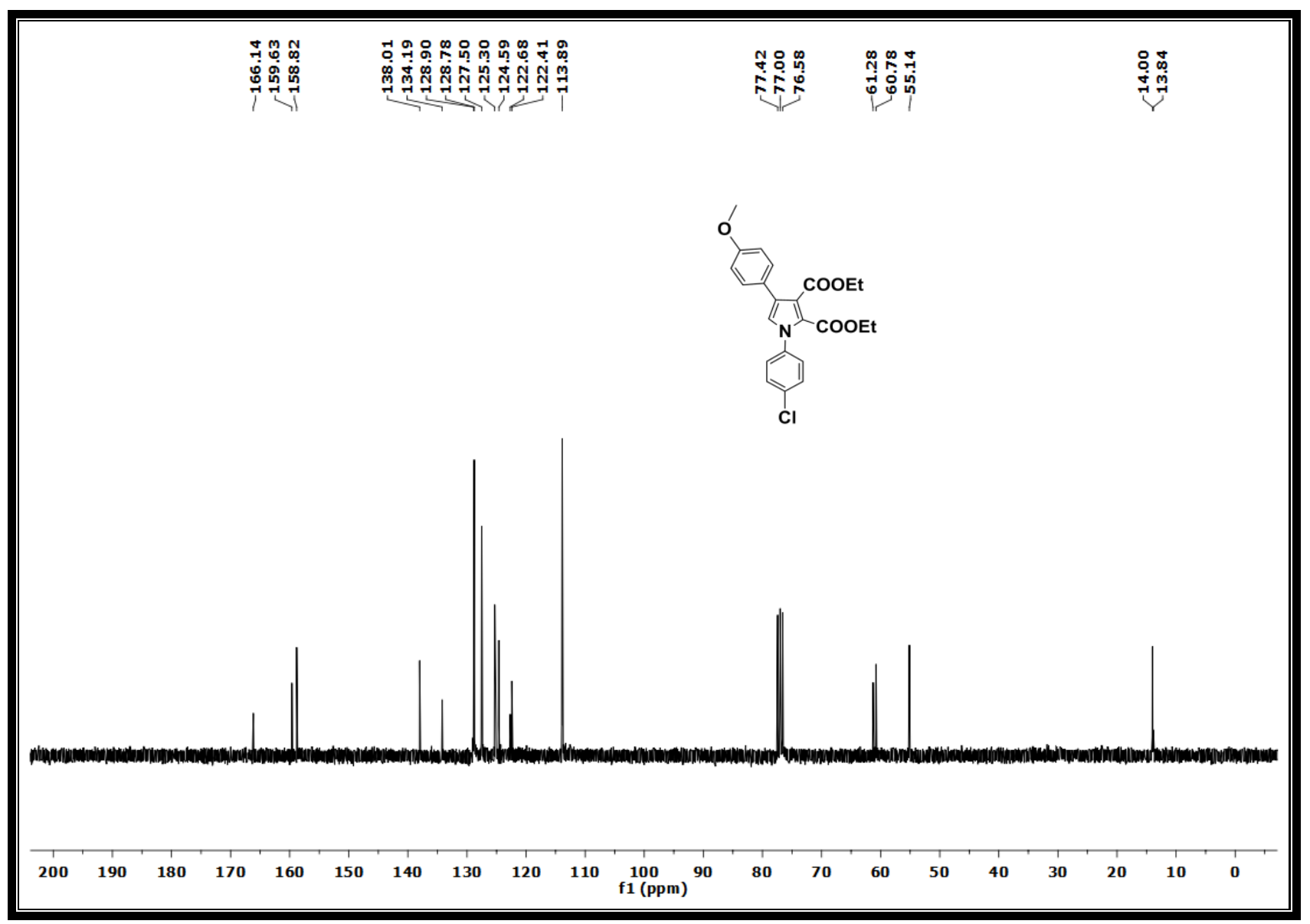

Fig.16. ${ }^{13} \mathrm{C}$ NMR spectrum $3 g$ 


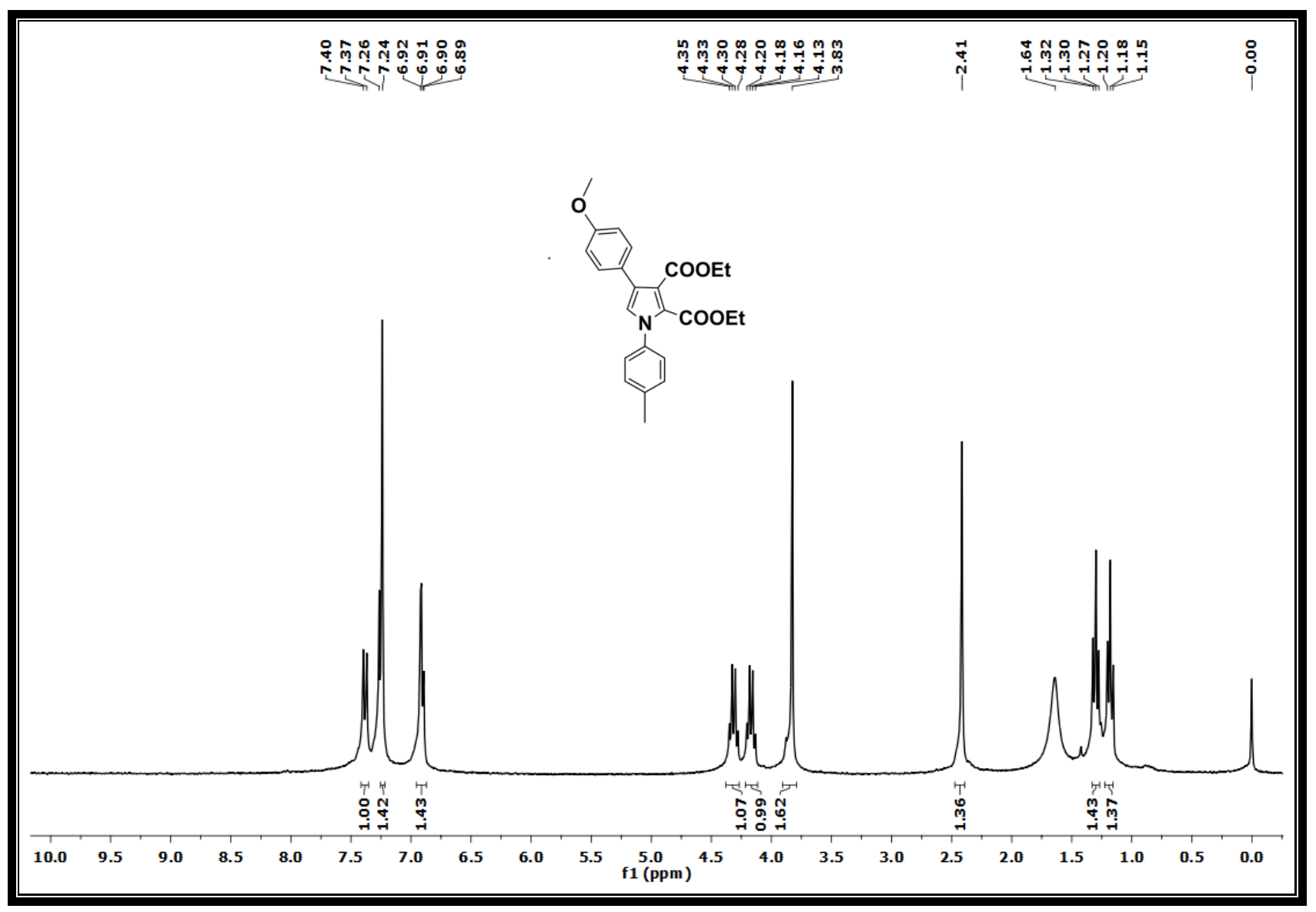

Fig. 17. ${ }^{1} \mathrm{H}$ NMR spectrum $\mathbf{3 h}$ 


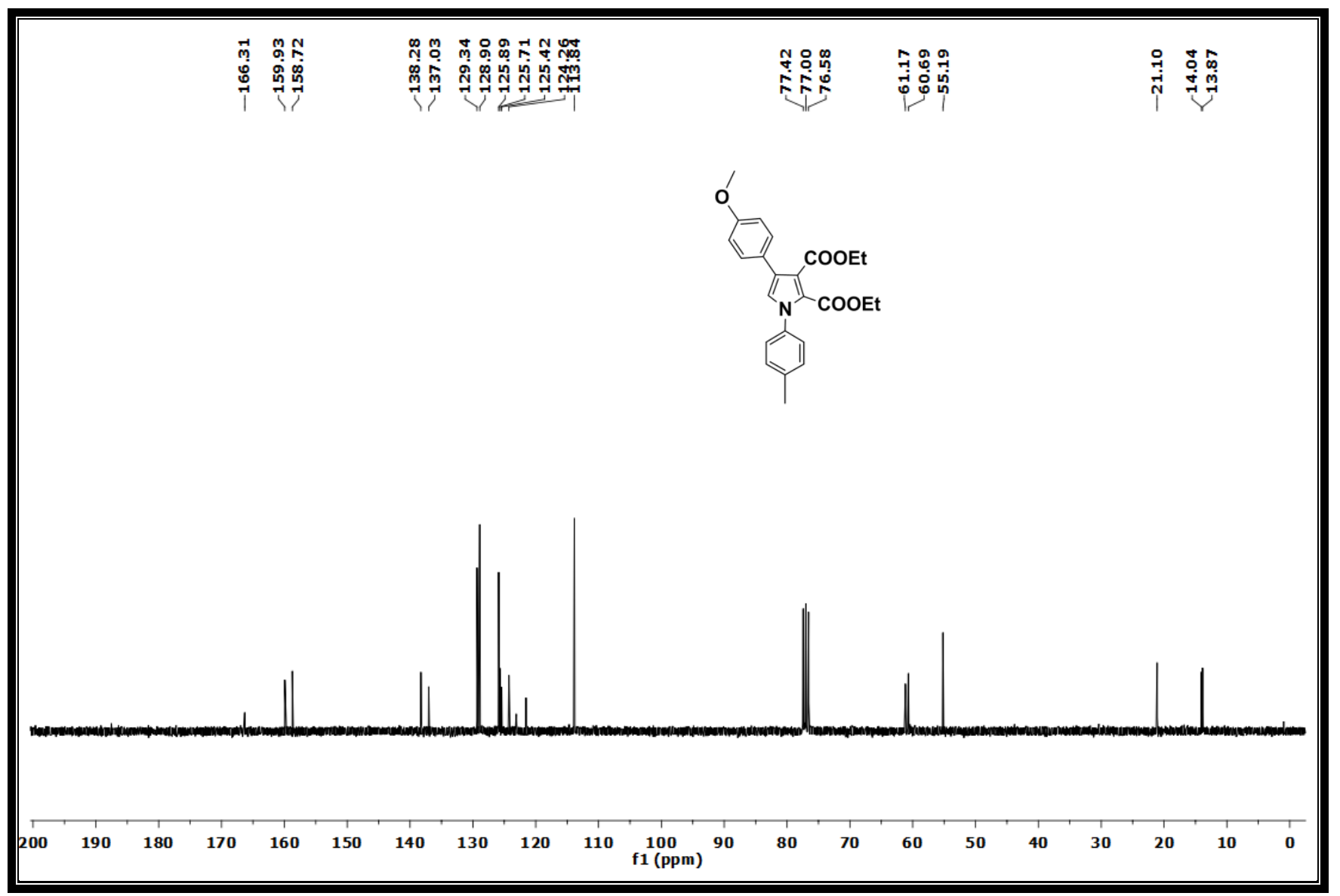

Fig.18. ${ }^{13} \mathrm{C}$ NMR spectrum $\mathbf{3 h}$ 


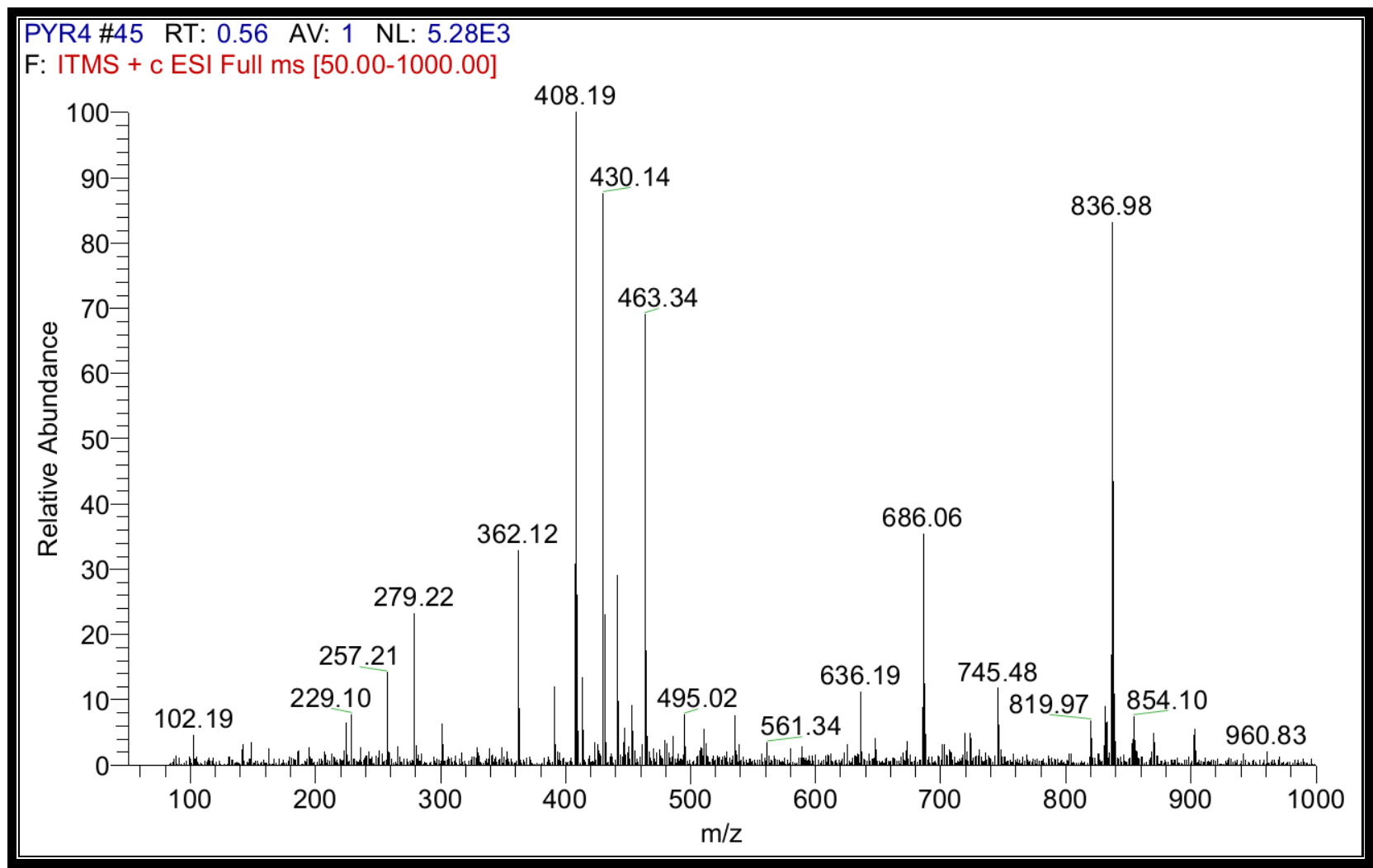

Fig.19. Mass spectrum of $\mathbf{3 h}$ 


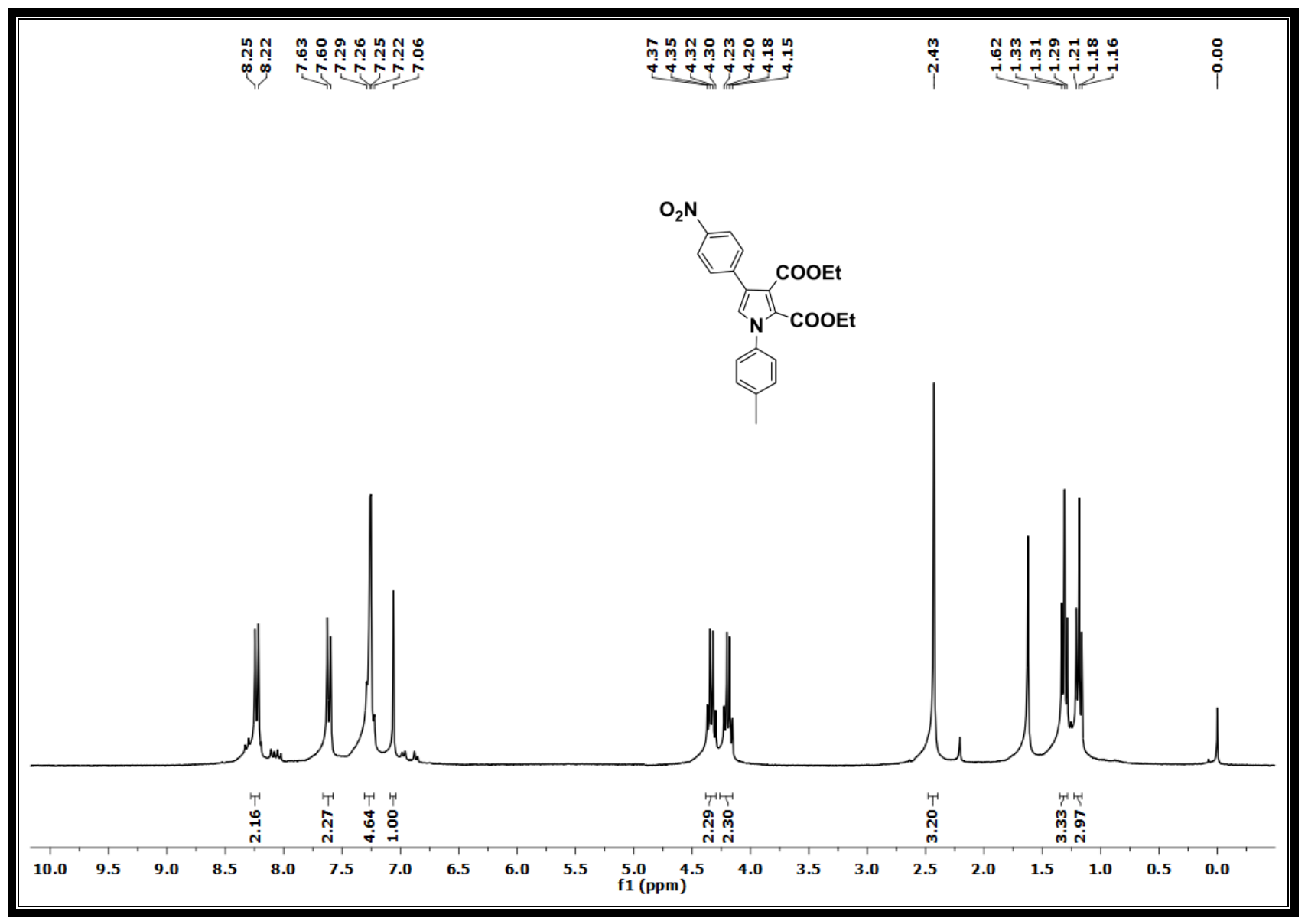

Fig.20. ${ }^{1} \mathrm{H}$ NMR spectrum 3i 


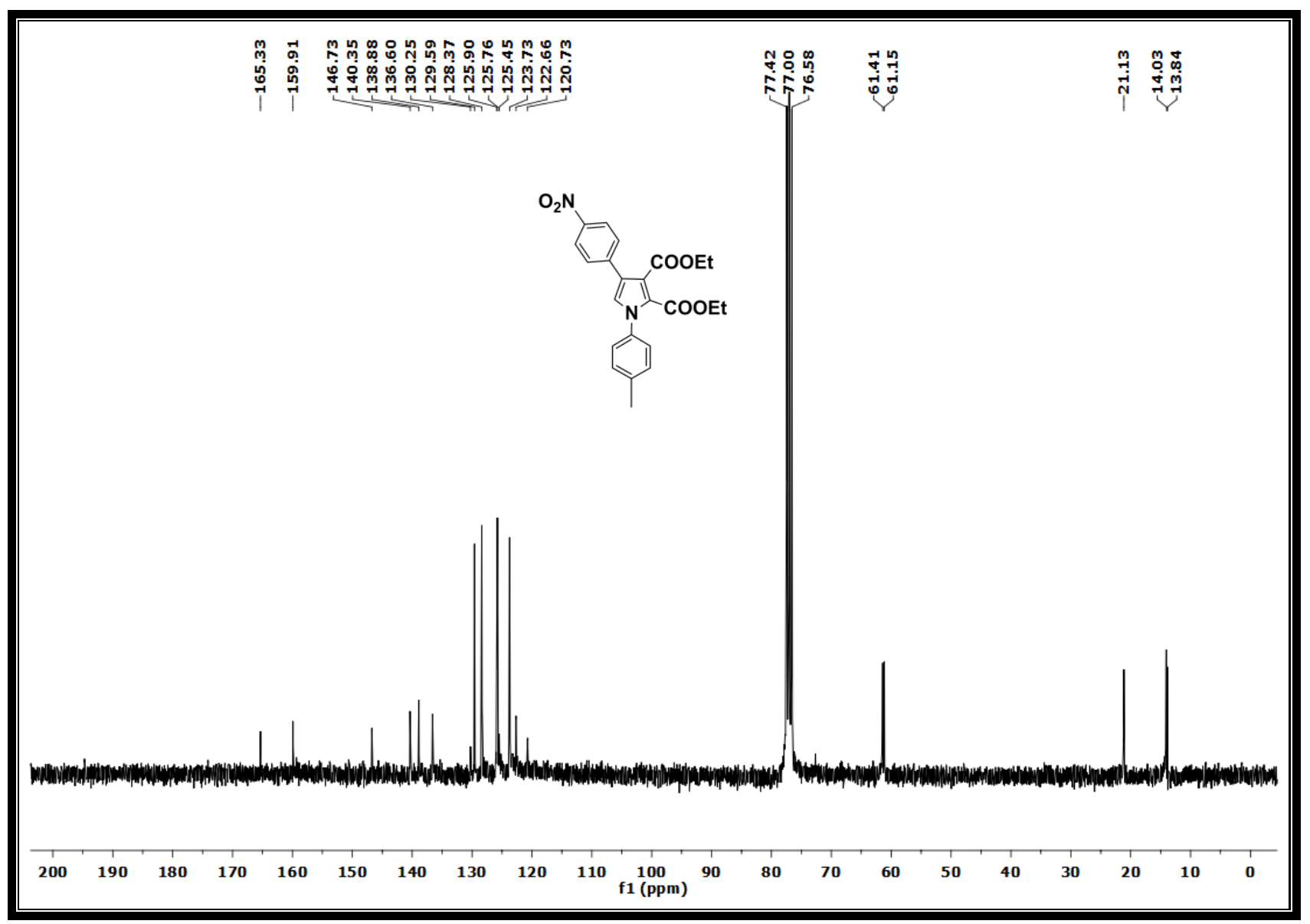

Fig. 21. ${ }^{13} \mathrm{C}$ NMR spectrum $3 \mathbf{i}$ 


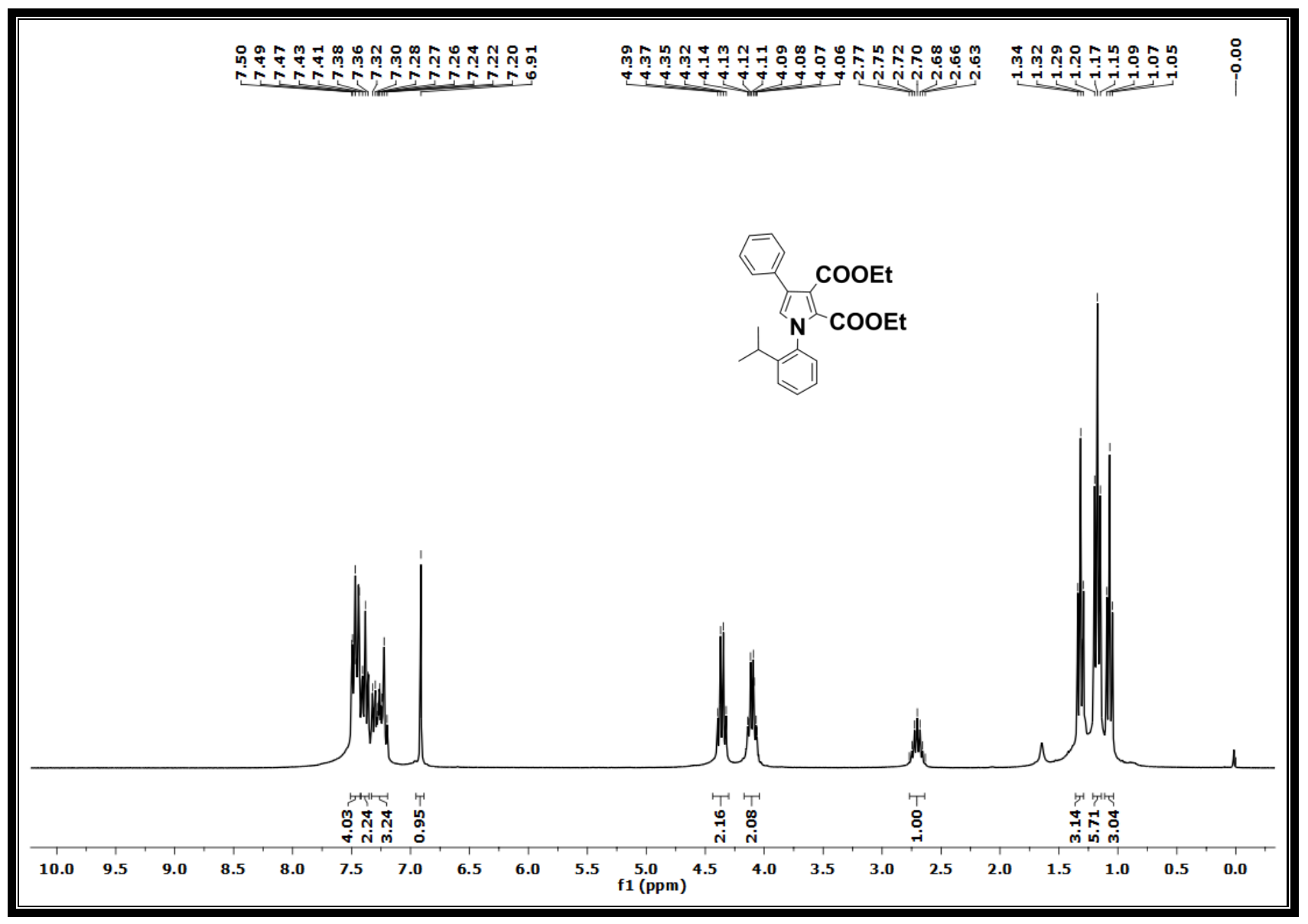

Fig.22. ${ }^{1} \mathrm{H}$ NMR spectrum $\mathbf{3 j}$ 


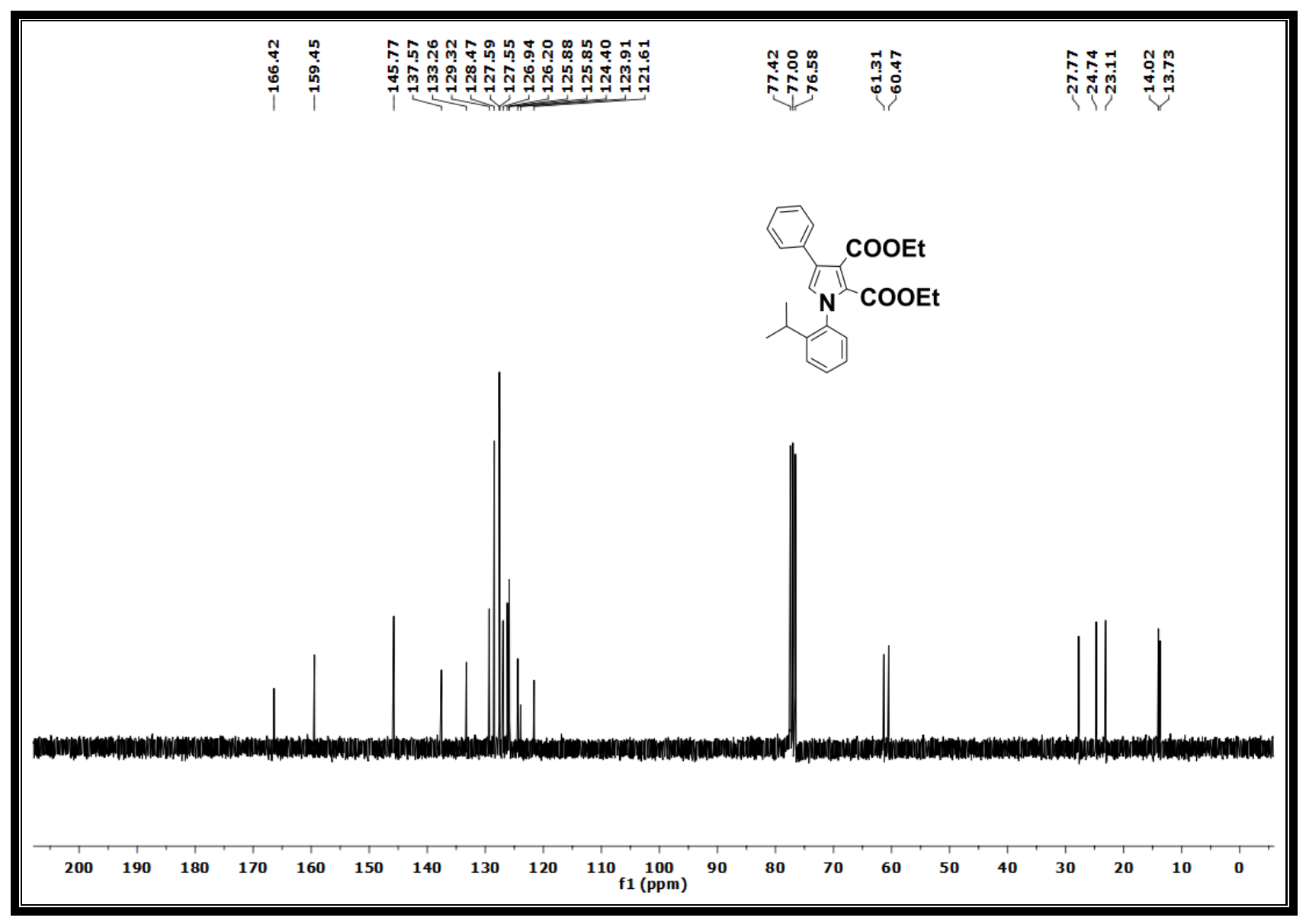

Fig.23. ${ }^{13} \mathrm{C}$ NMR spectrum $\mathbf{3 j}$ 


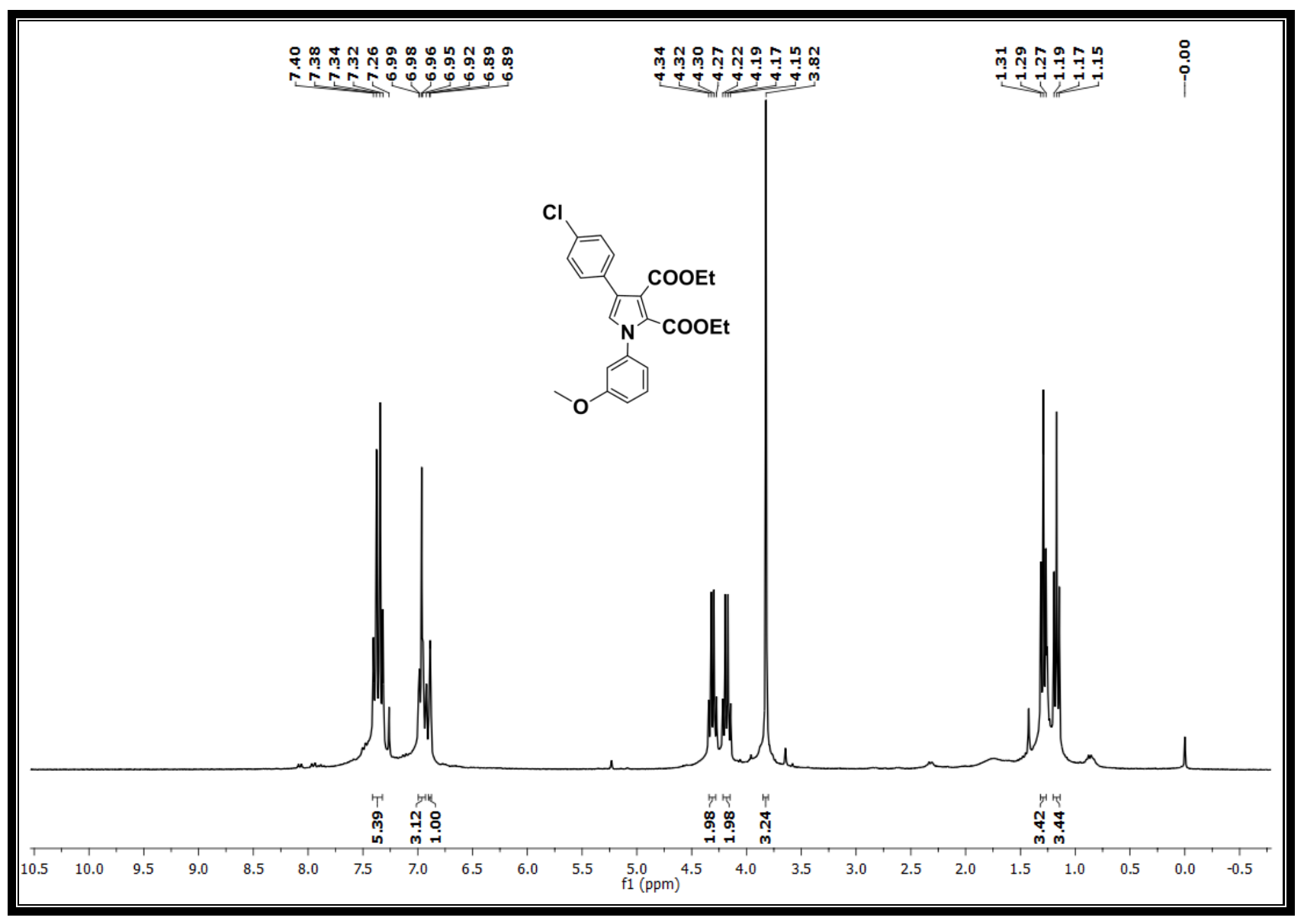

Fig. 24. ${ }^{1} \mathrm{H}$ NMR spectrum $\mathbf{3 k}$ 


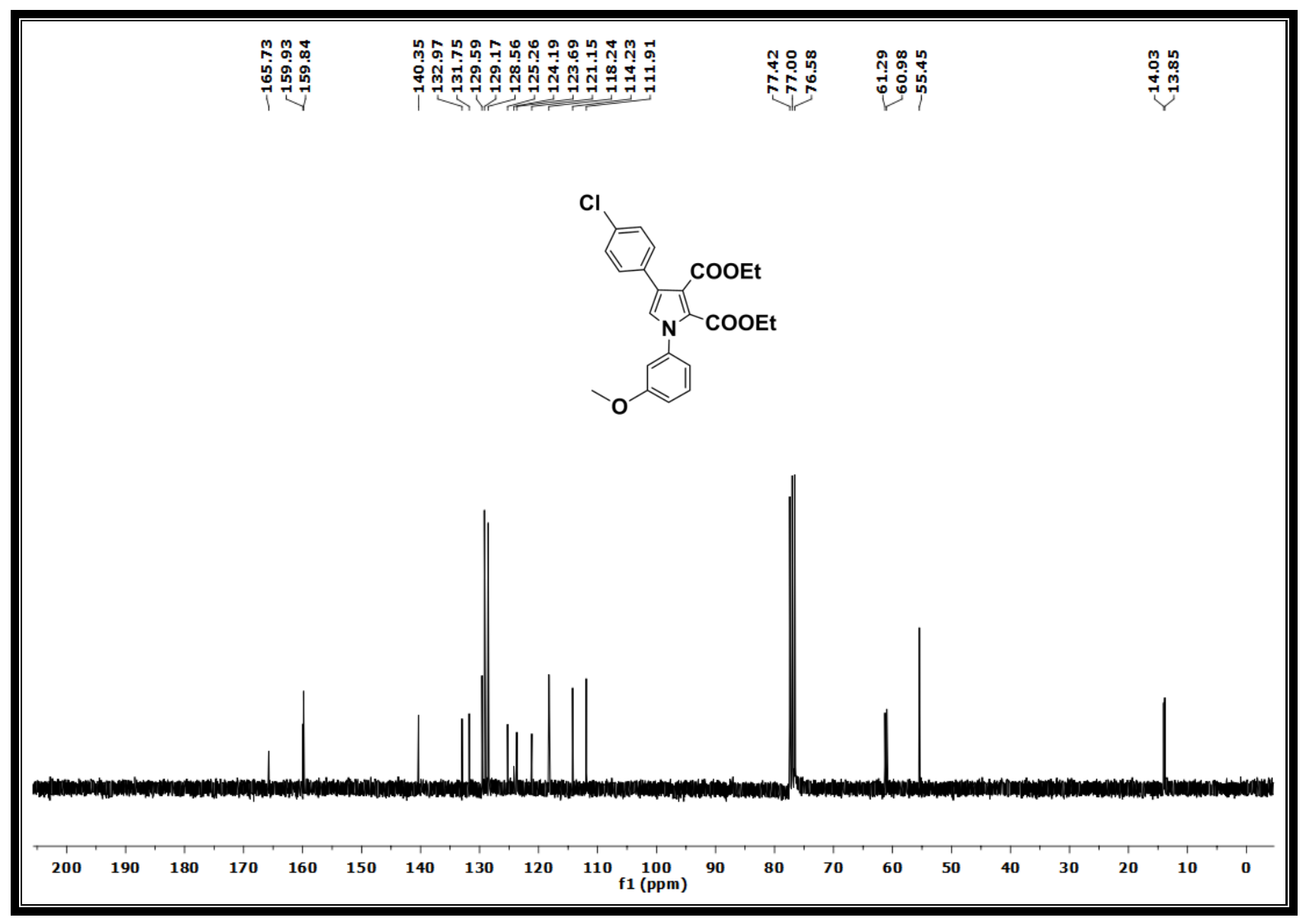

Fig. 25. ${ }^{13} \mathrm{C}$ NMR spectrum $\mathbf{3 k}$ 


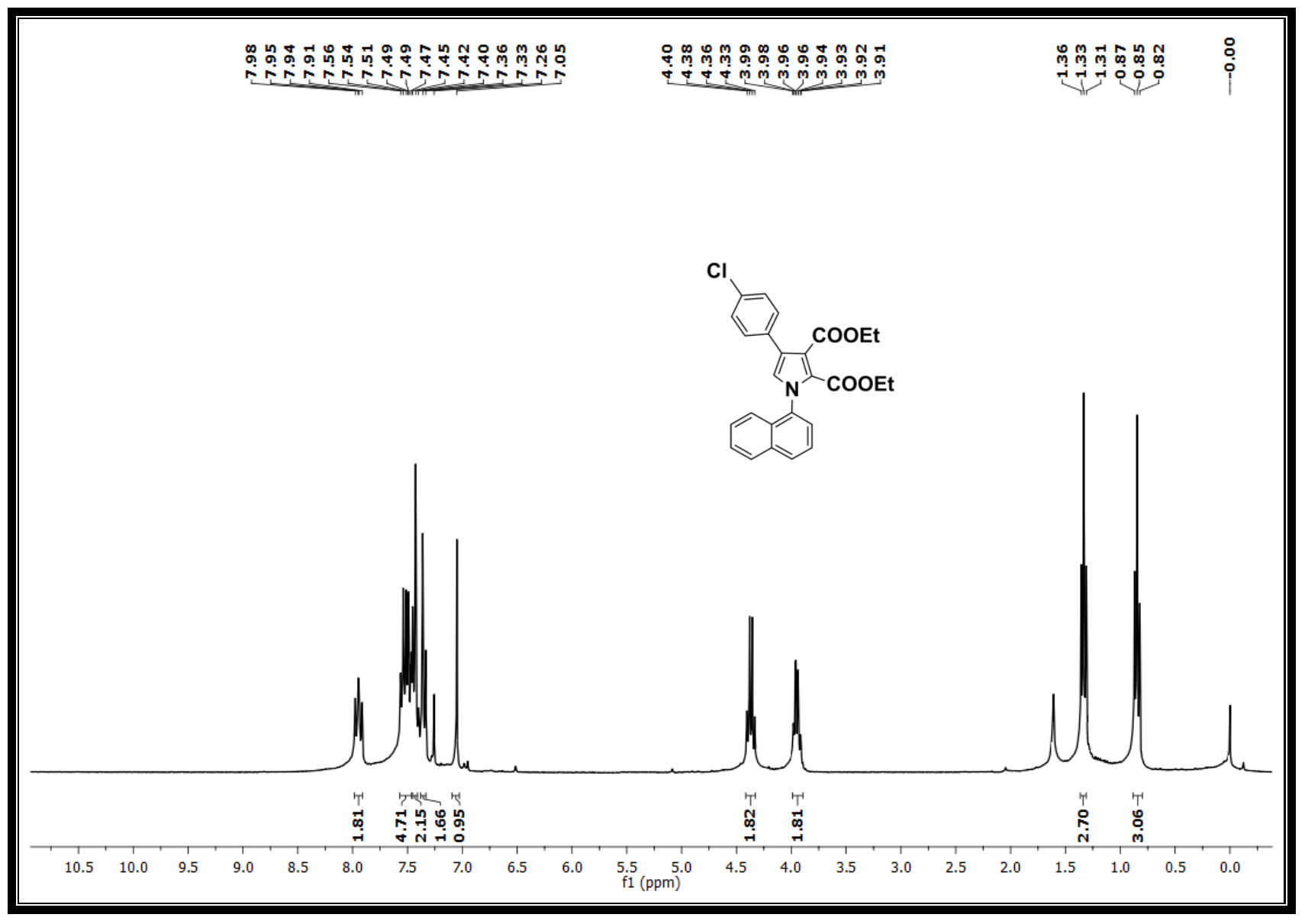

Fig.26. ${ }^{1} \mathrm{H}$ NMR spectrum 3 I 


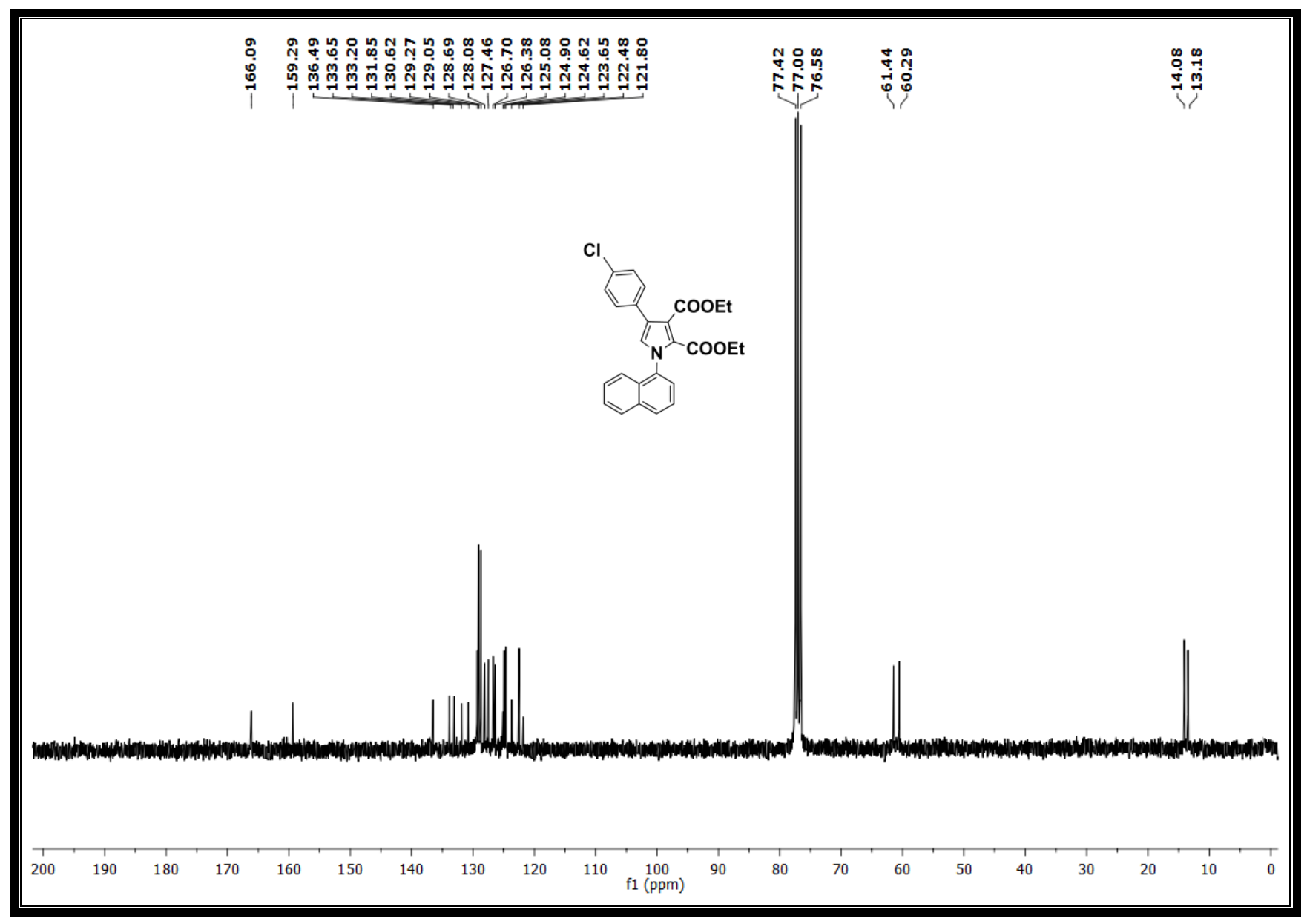

Fig.27. ${ }^{13} \mathrm{C}$ NMR spectrum 31 


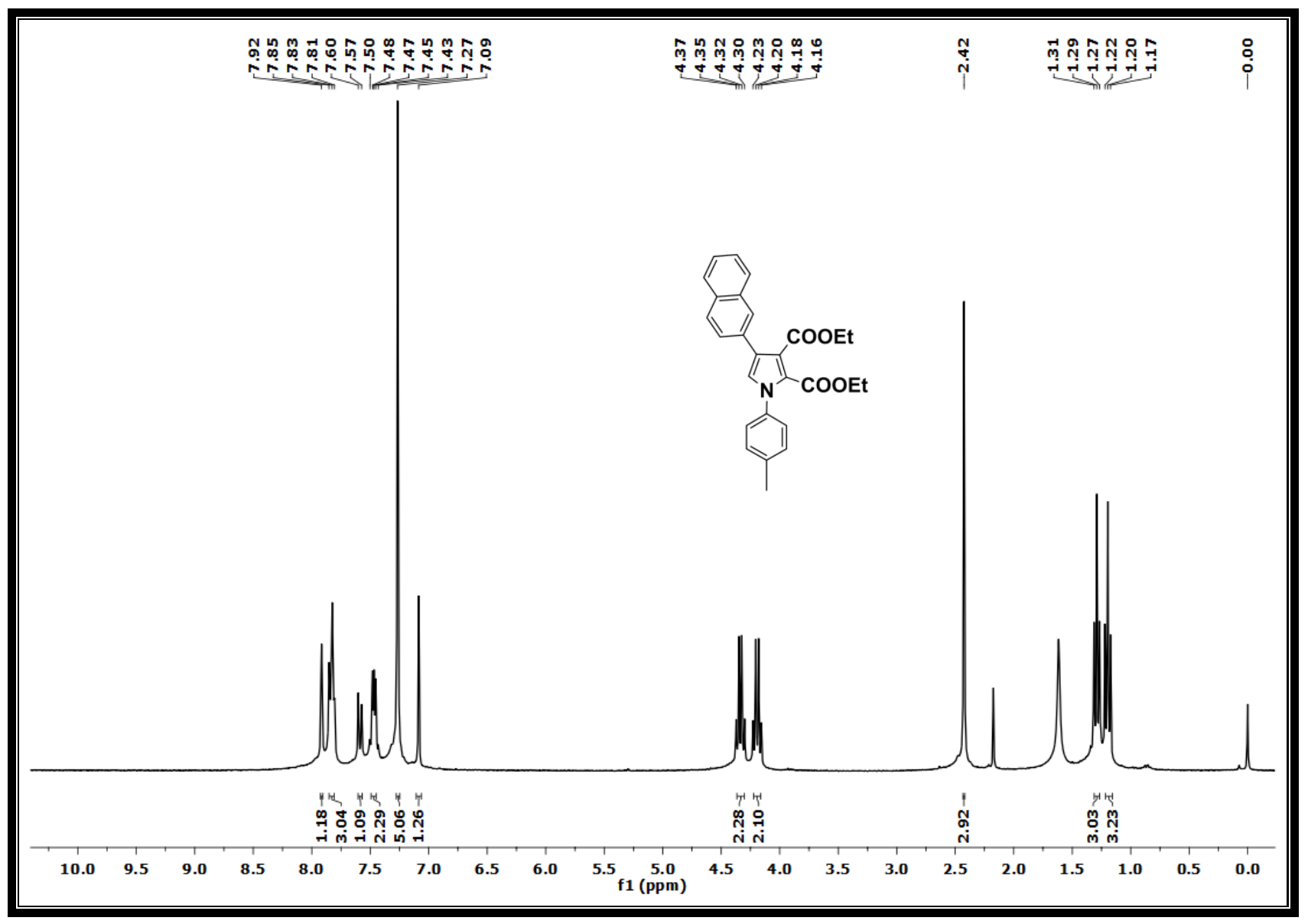

Fig.28. ${ }^{1}$ H NMR spectrum $\mathbf{3 m}$ 


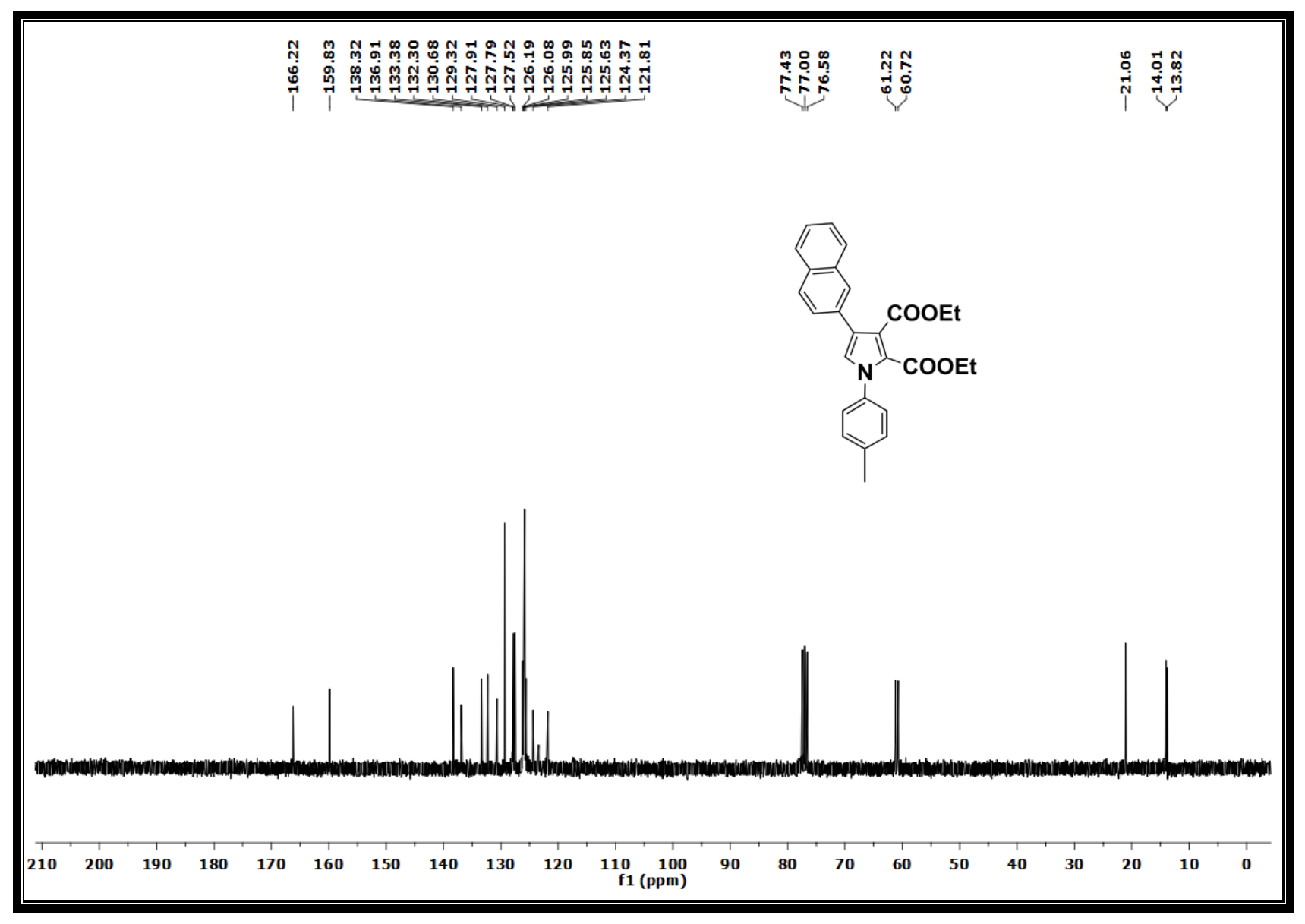

Fig.29. ${ }^{13} \mathrm{C}$ NMR spectrum $\mathbf{3 m}$ 


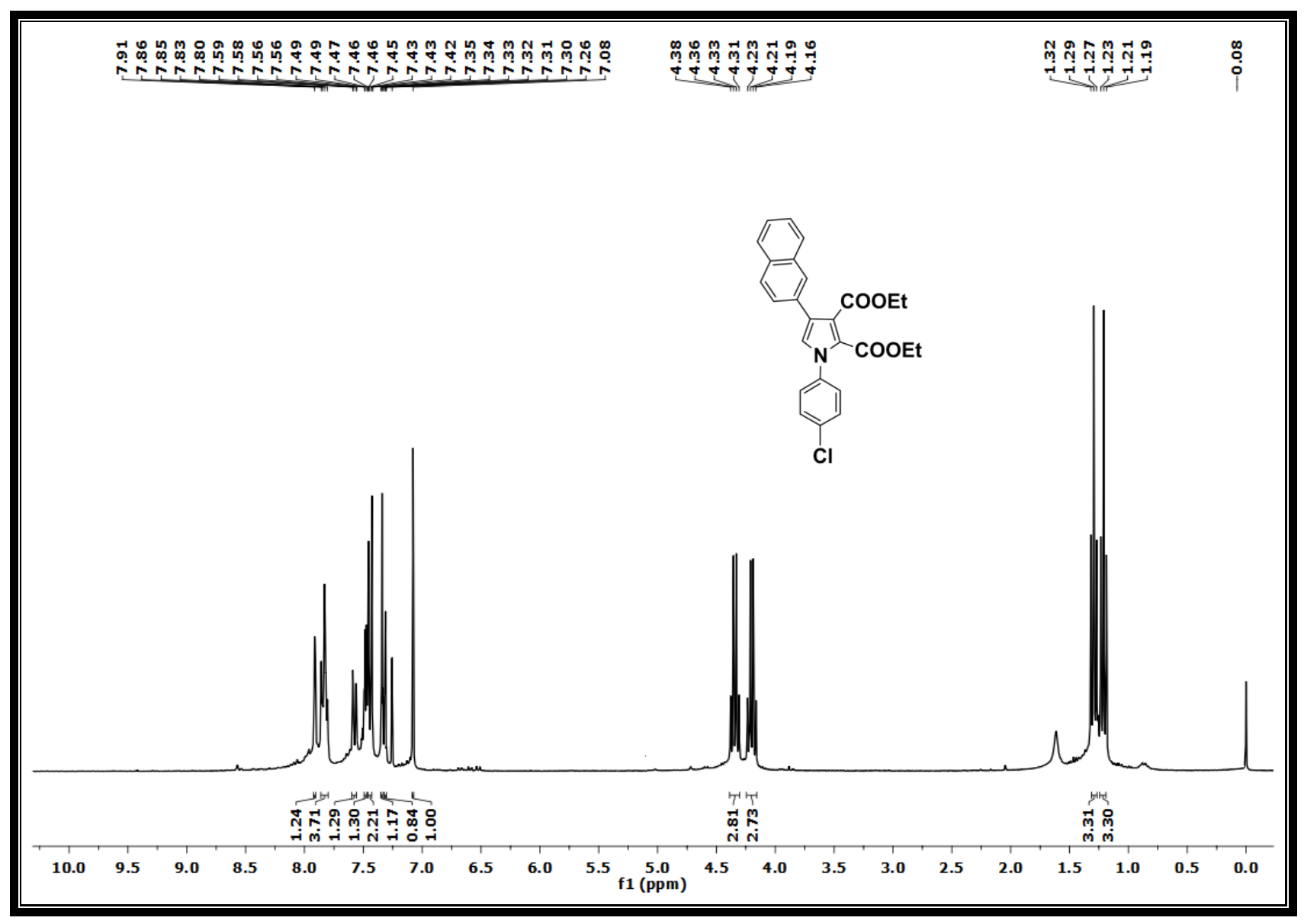

Fig.30. ${ }^{1}$ H NMR spectrum 3n 


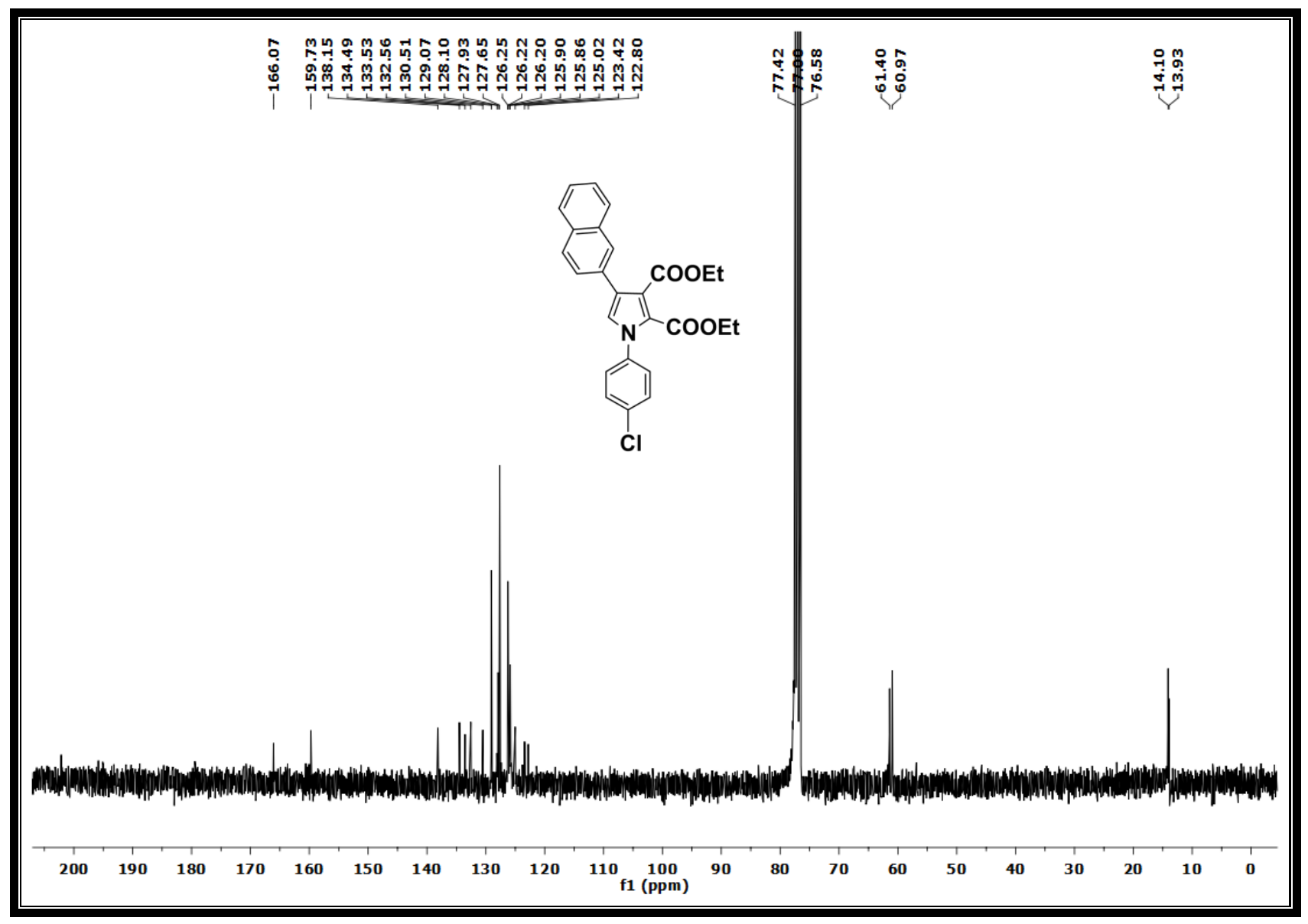

Fig. $31 .{ }^{13}$ C NMR spectrum 3n 


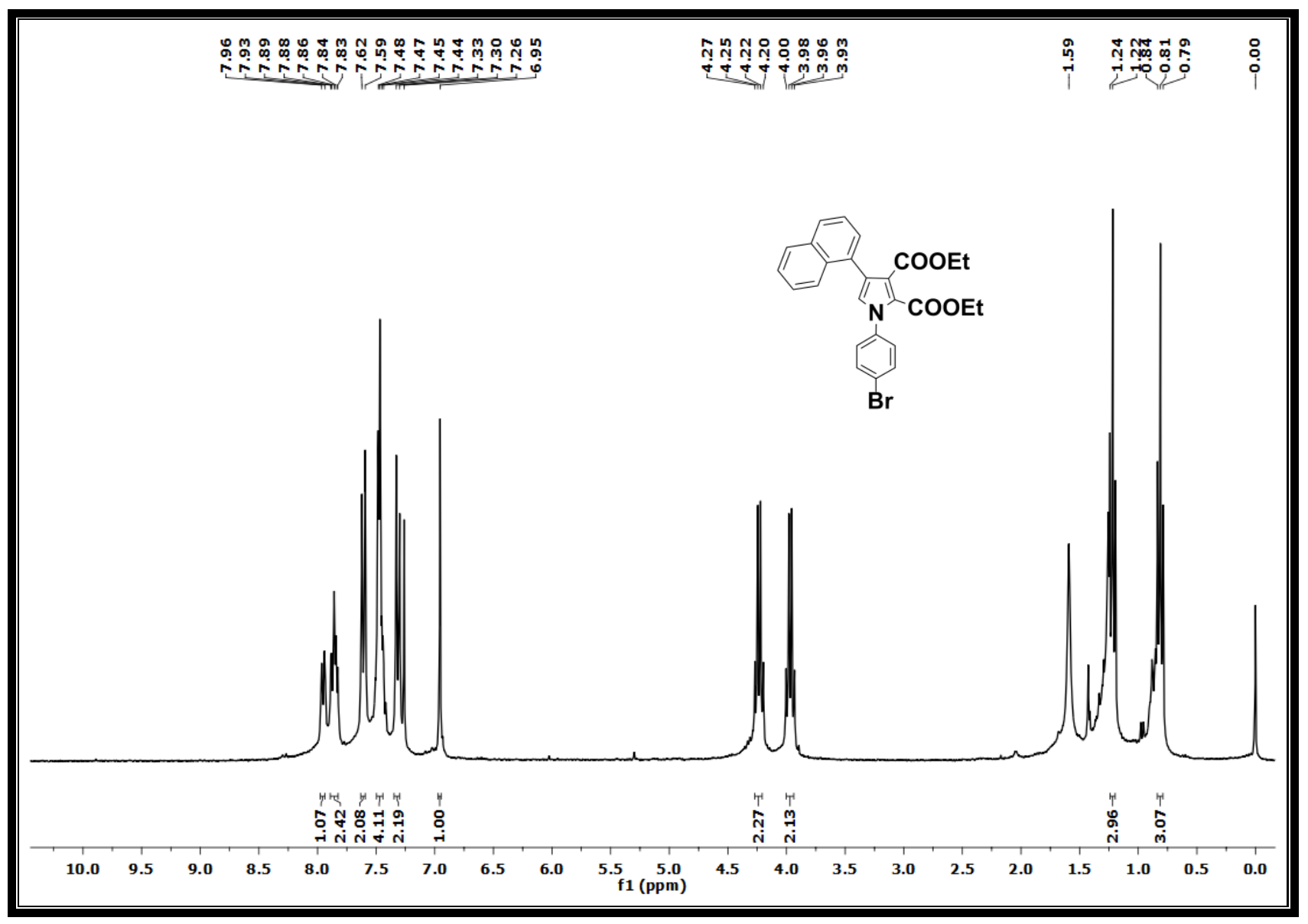

Fig.32. ${ }^{1} \mathrm{H}$ NMR spectrum 30 


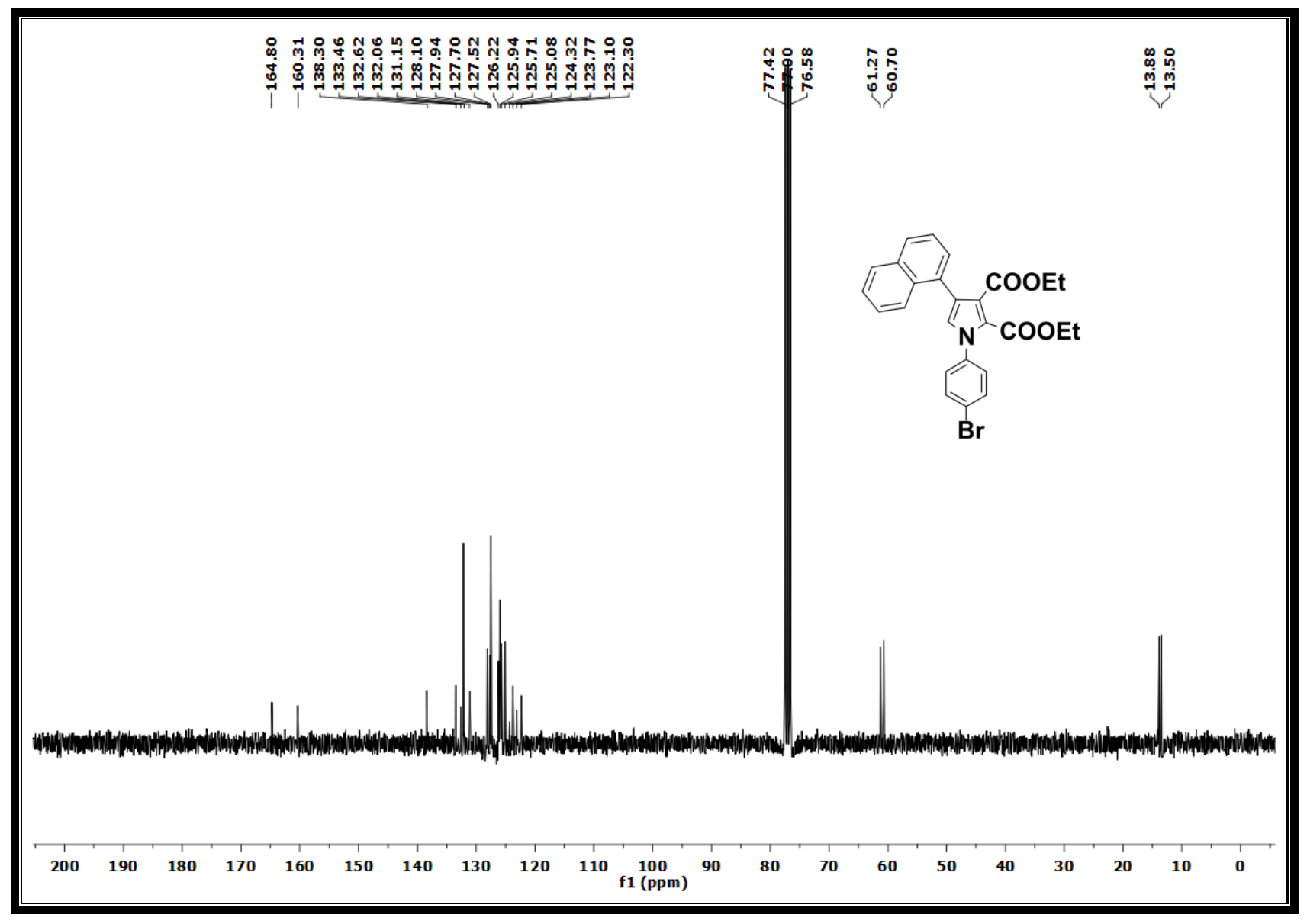

Fig.33. ${ }^{13} \mathrm{C}$ NMR spectrum 30 


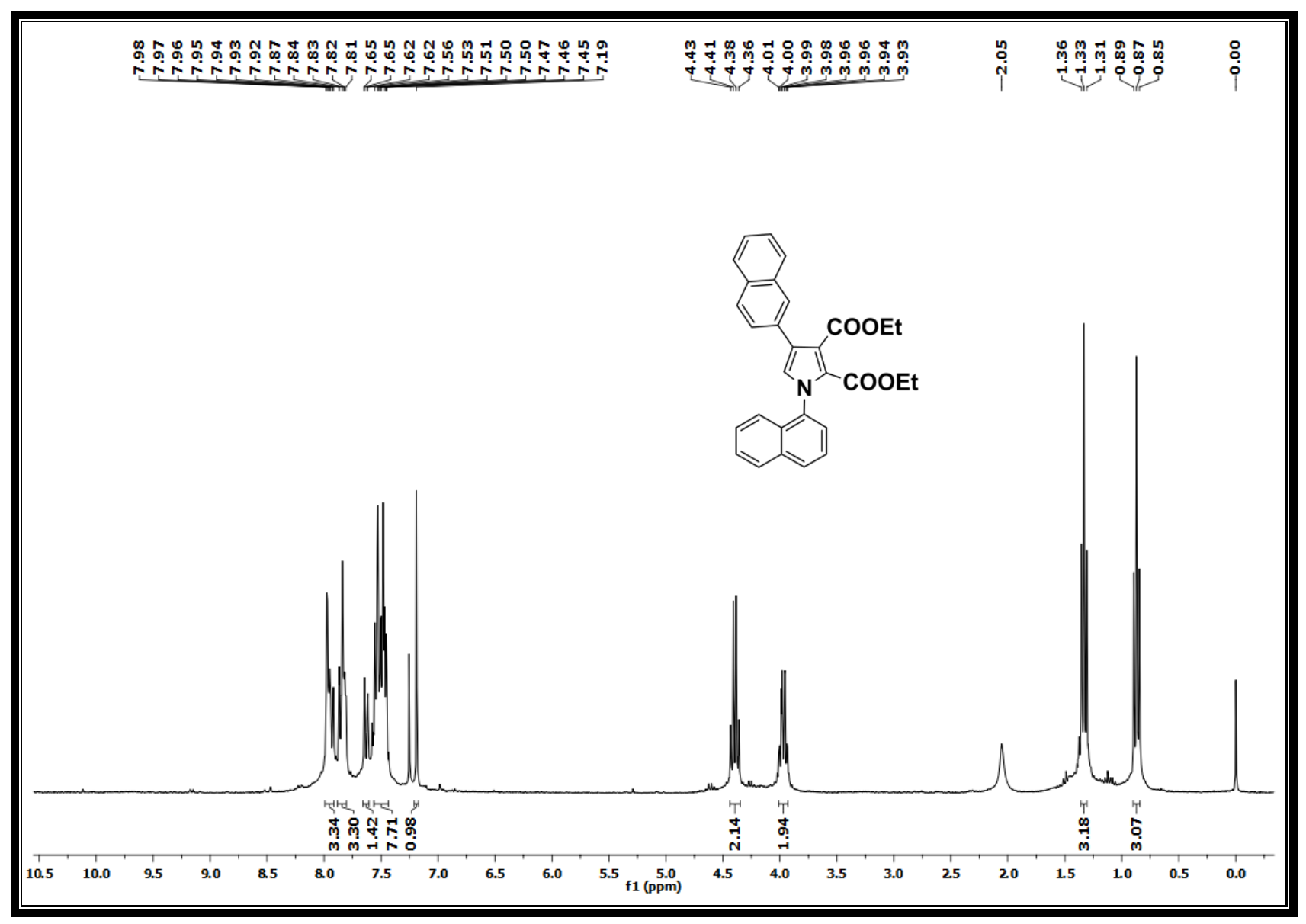

Fig. $34 .{ }^{1} \mathrm{H}$ NMR spectrum $\mathbf{3 p}$ 


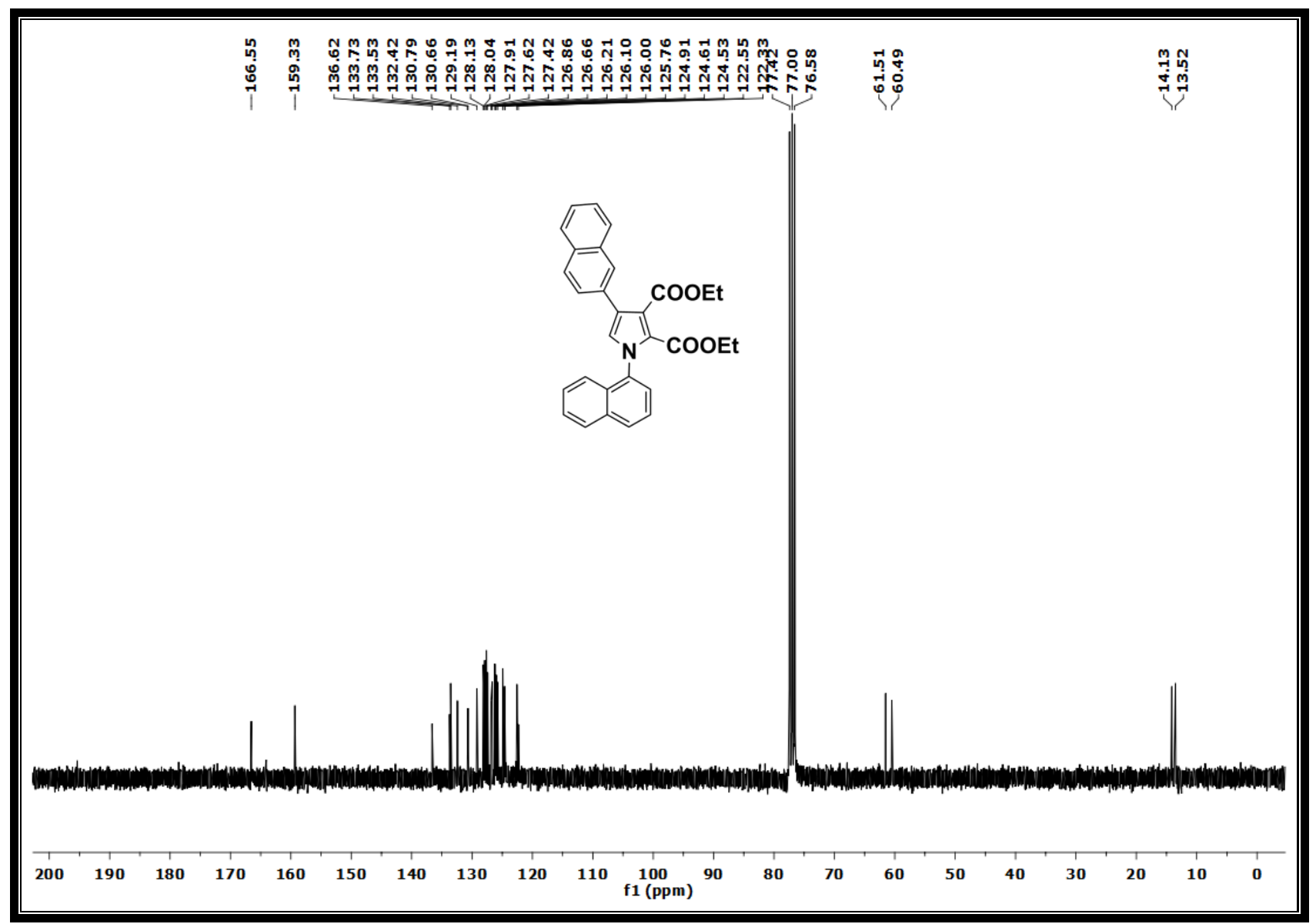

Fig. 35. ${ }^{13}$ C NMR spectrum 3p 


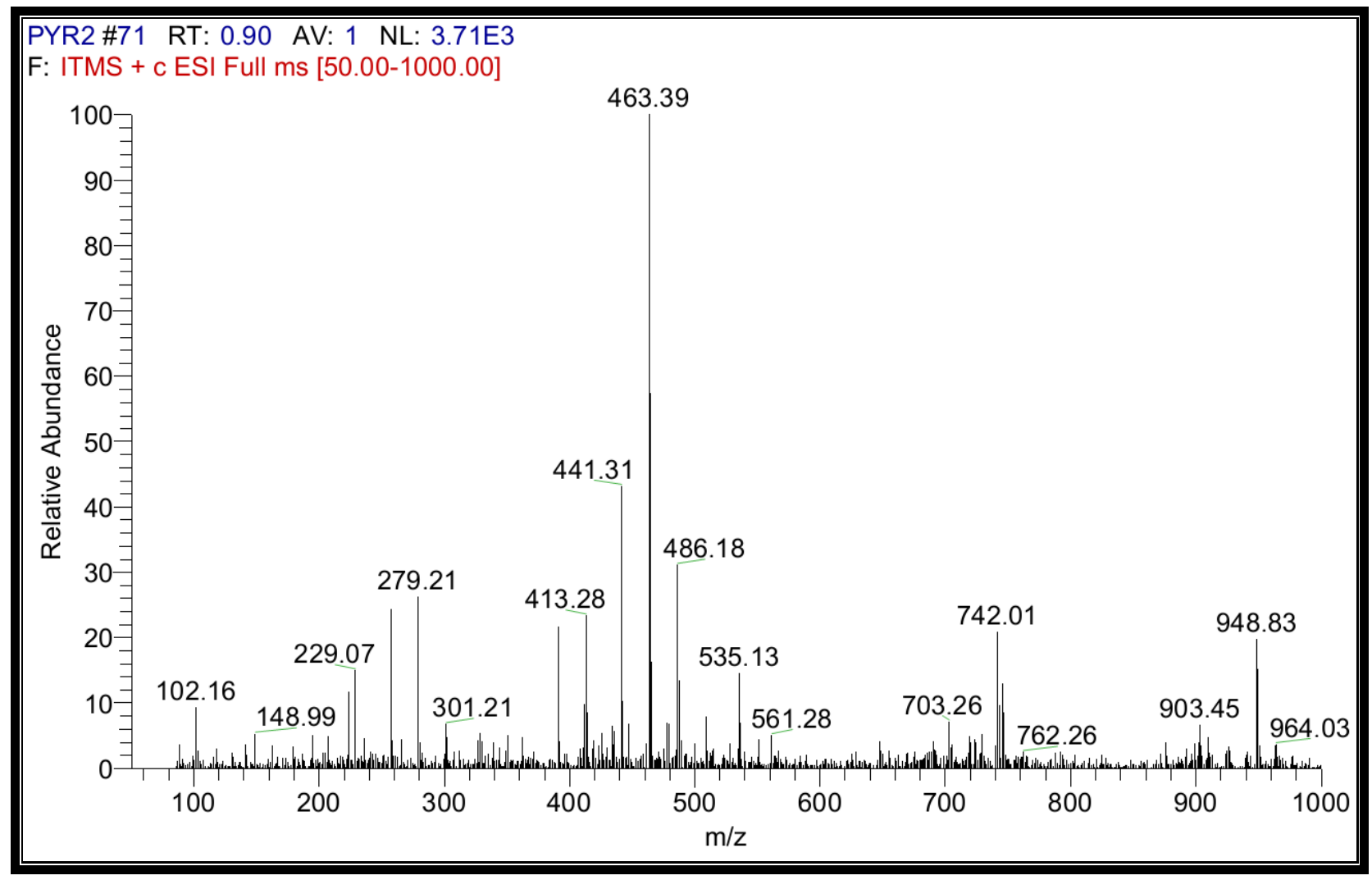

Fig.36. Mass spectrum of $\mathbf{3 p}$ 


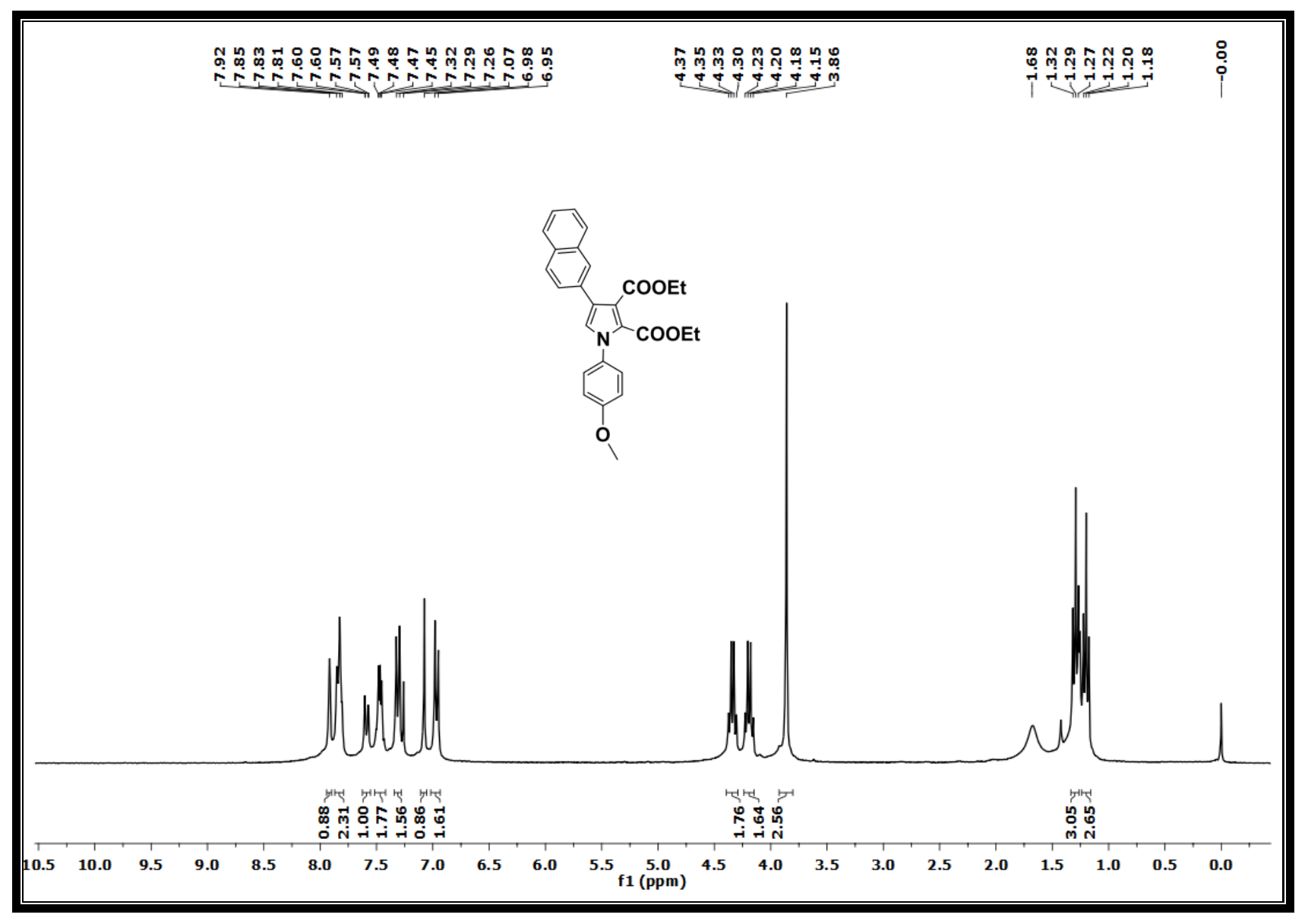

Fig. 37. ${ }^{1}$ H NMR spectrum $\mathbf{3 q}$ 


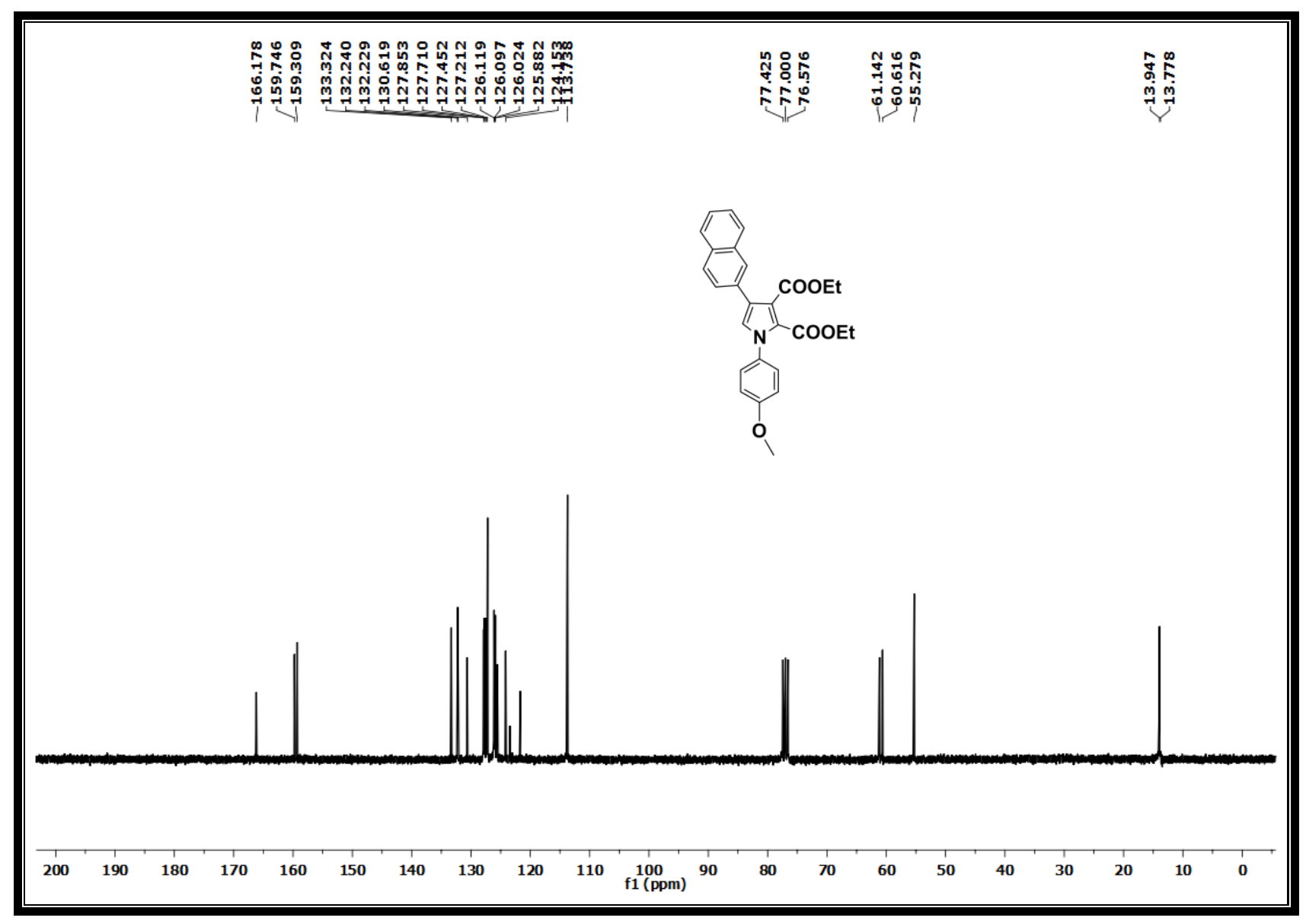

Fig. 38. ${ }^{13}$ C NMR spectrum $\mathbf{3 q}$ 


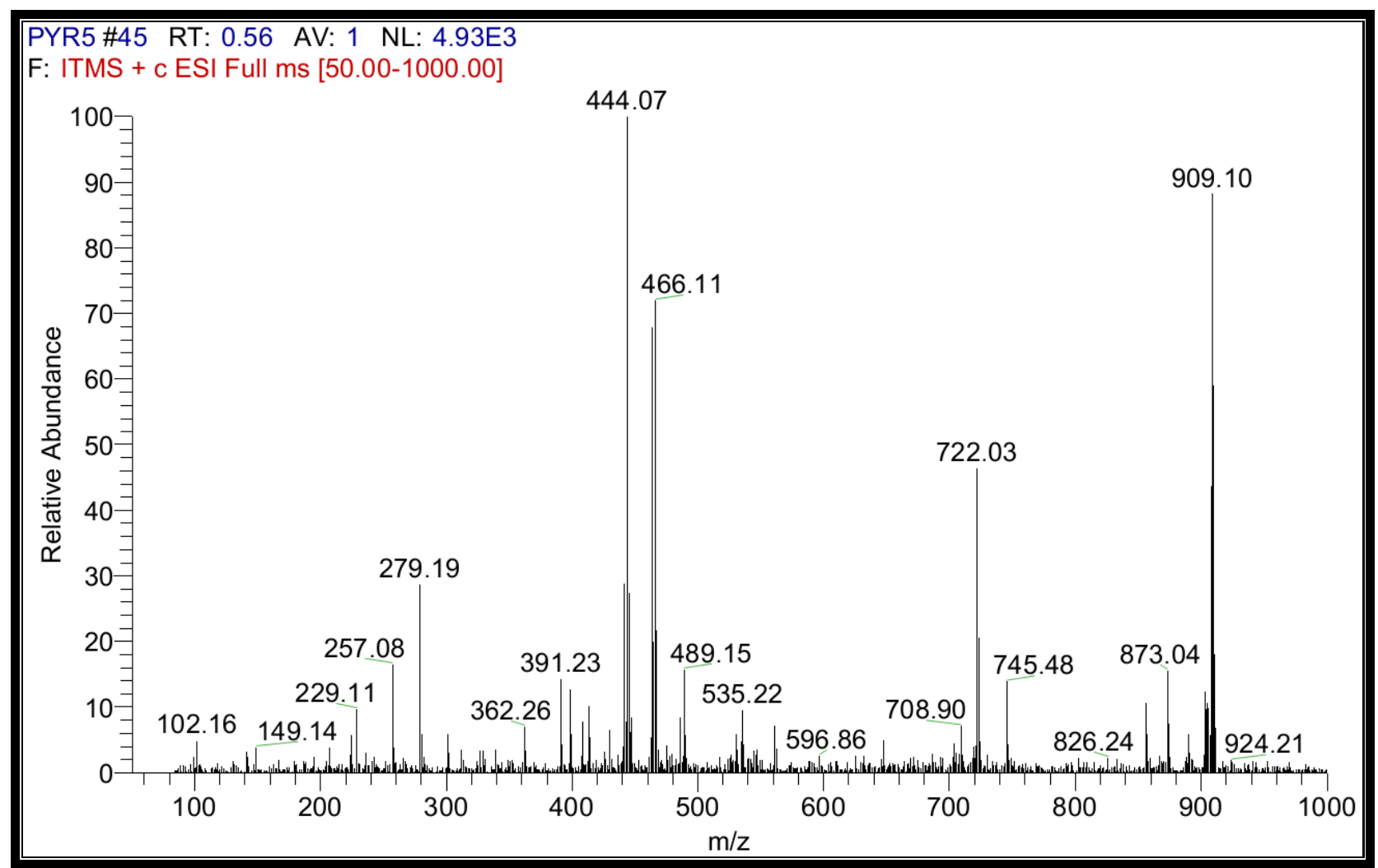

Fig.39. Mass spectrum of $\mathbf{3 q}$ 


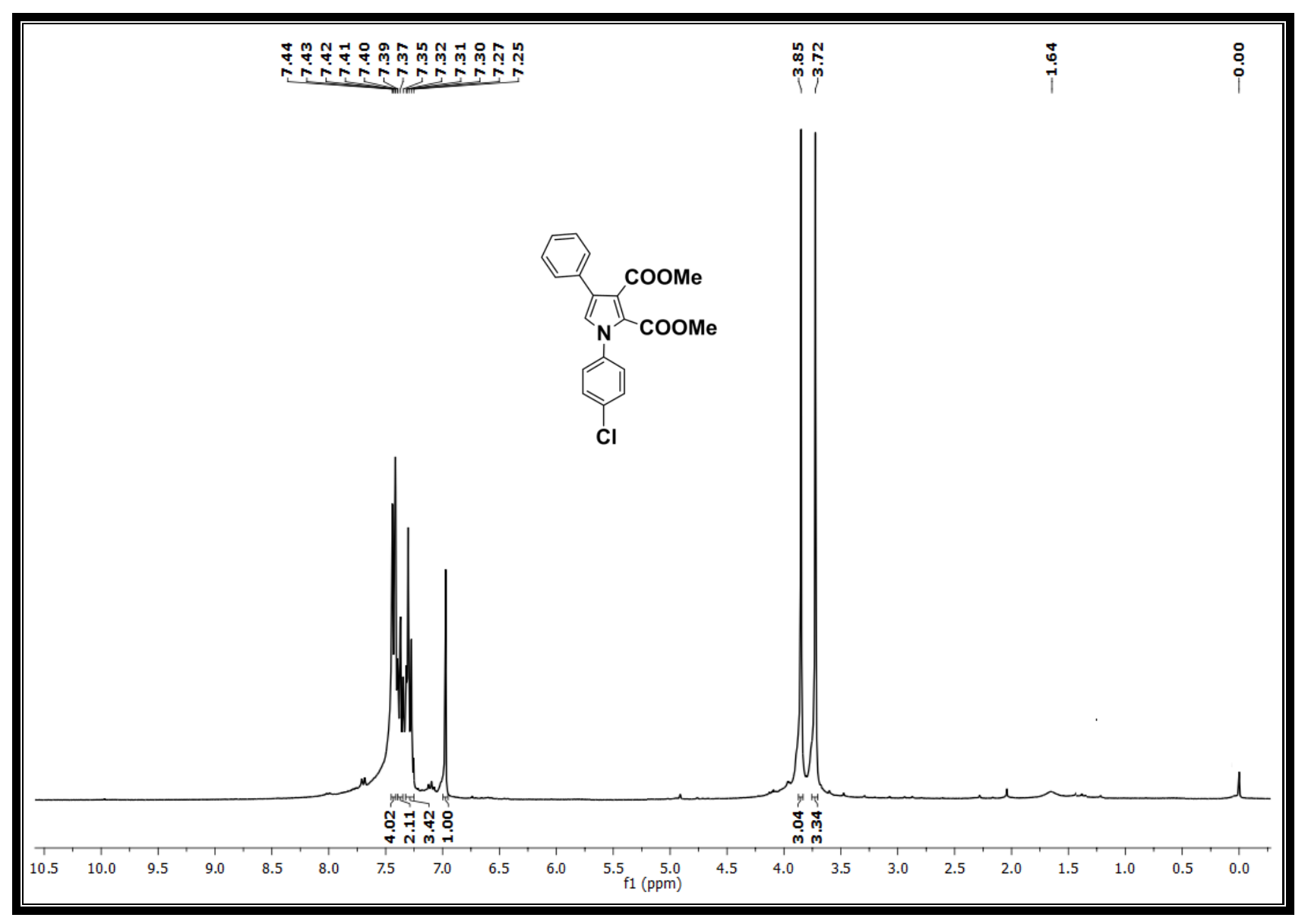

Fig.40. ${ }^{1}$ H NMR spectrum $3 \mathbf{r}$ 


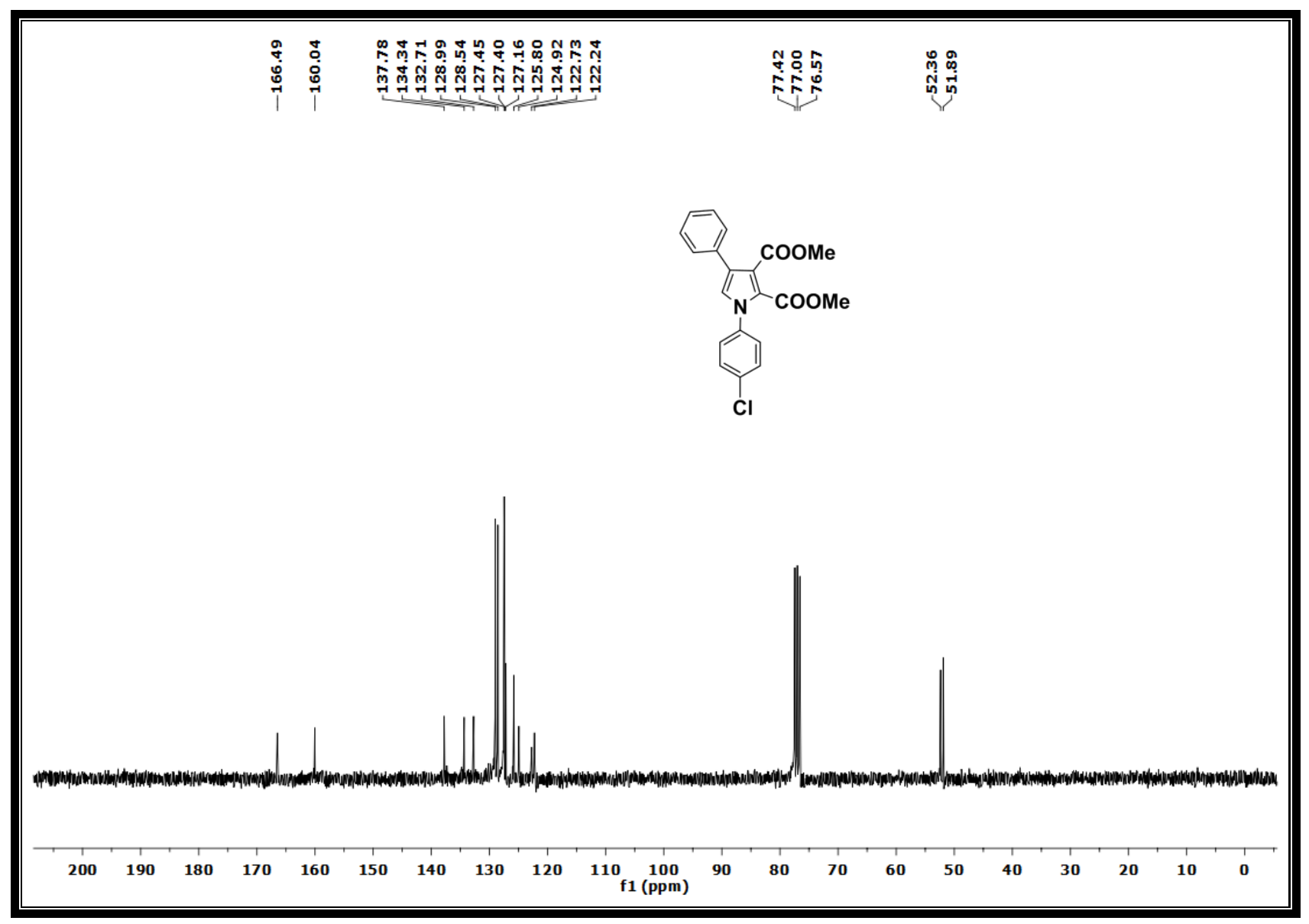

Fig. $41 .{ }^{13} \mathrm{C}$ NMR spectrum $3 \mathbf{r}$ 


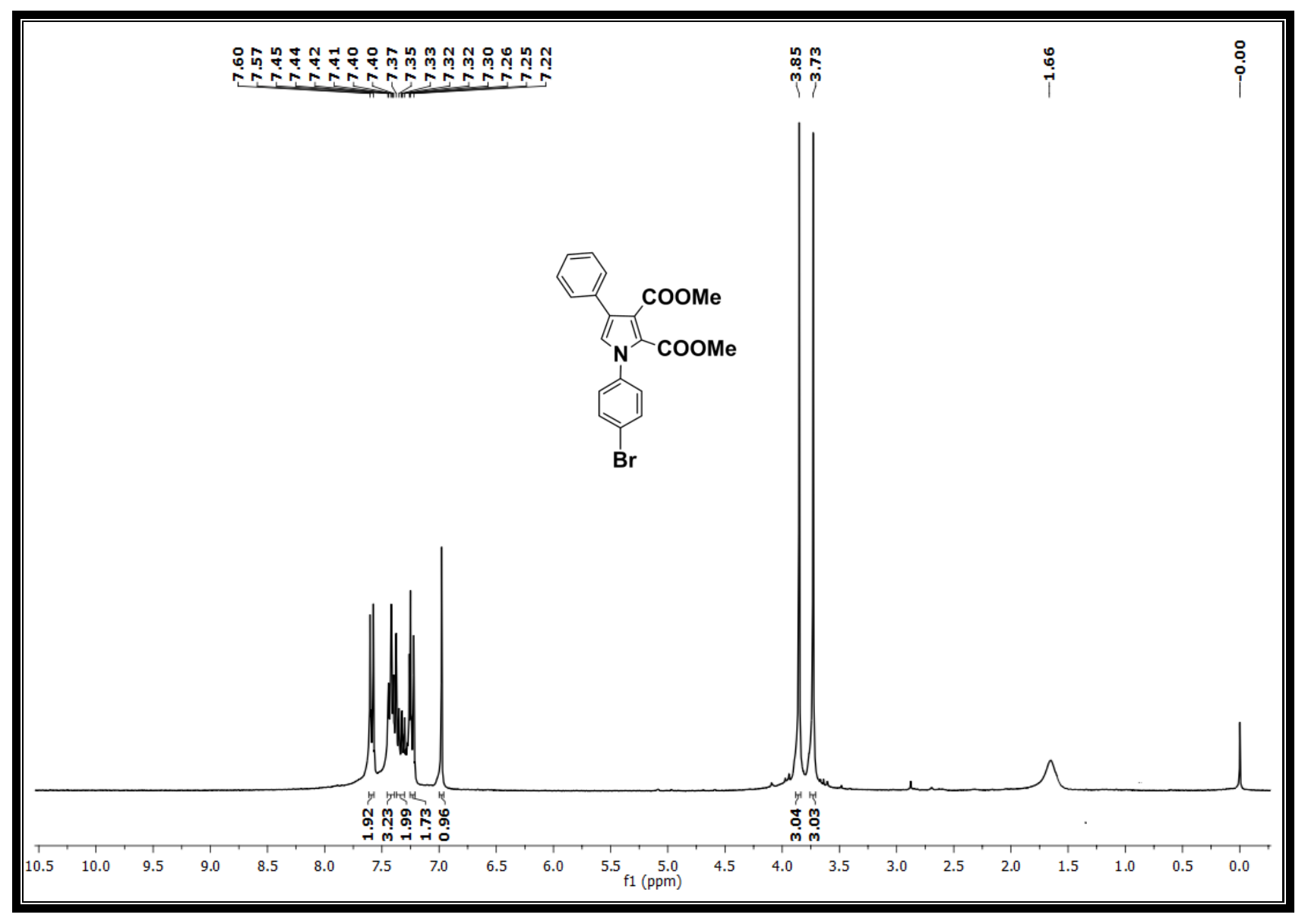

Fig.42. ${ }^{1} \mathrm{H}$ NMR spectrum 3 s 


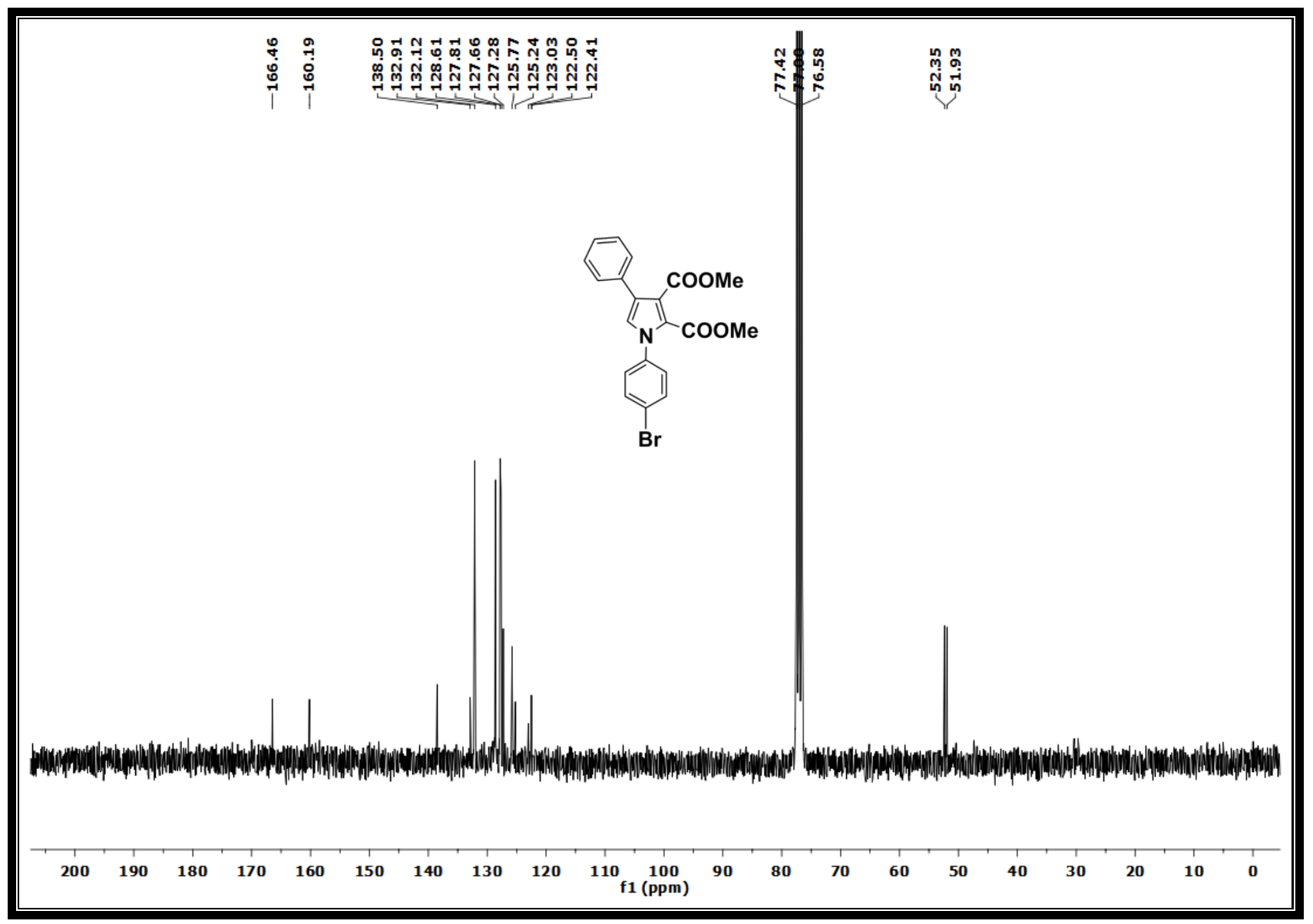

Fig.43. ${ }^{13} \mathrm{C}$ NMR spectrum 3 s 


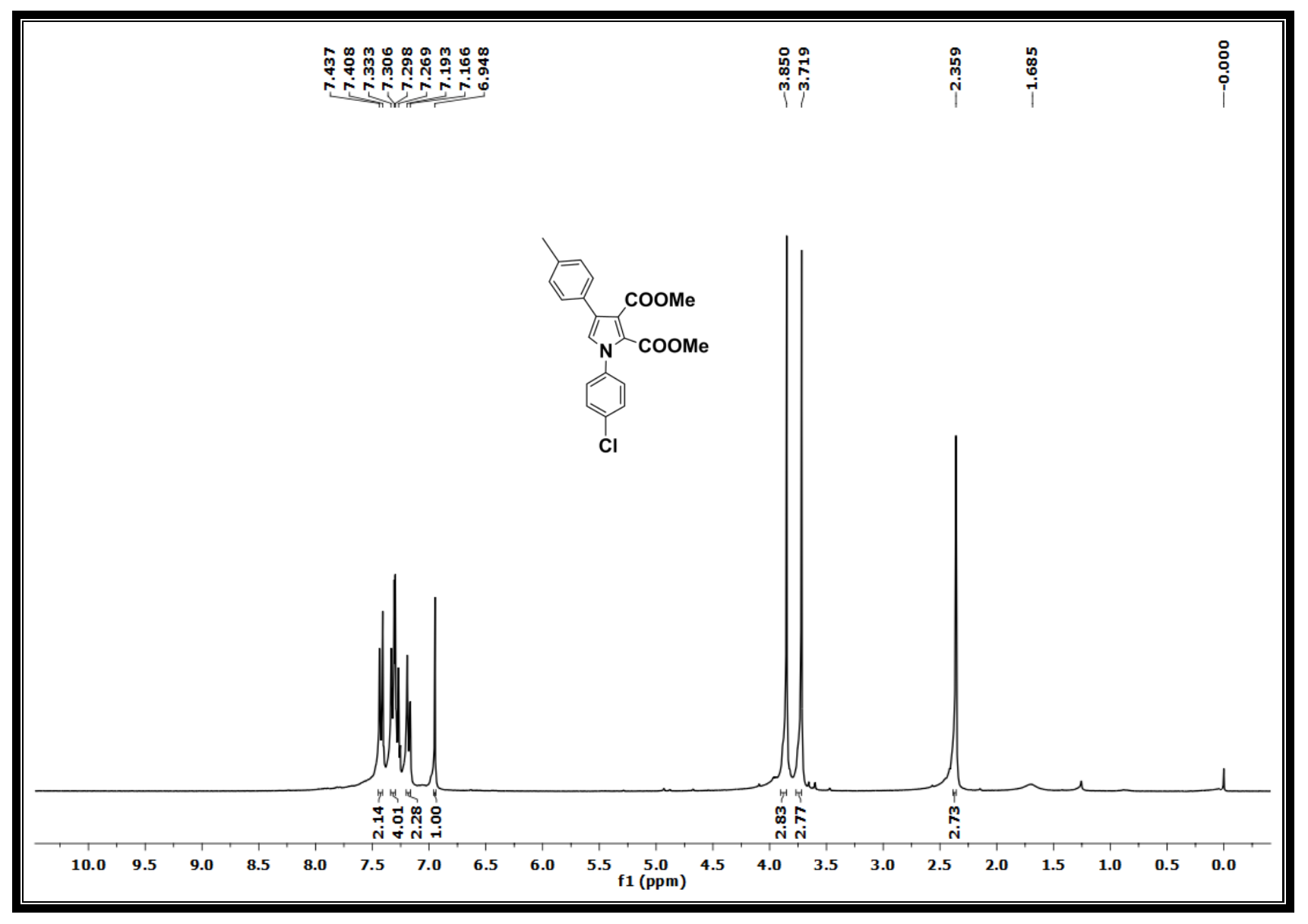

Fig.44. ${ }^{1} \mathrm{H}$ NMR spectrum $3 \mathbf{t}$ 


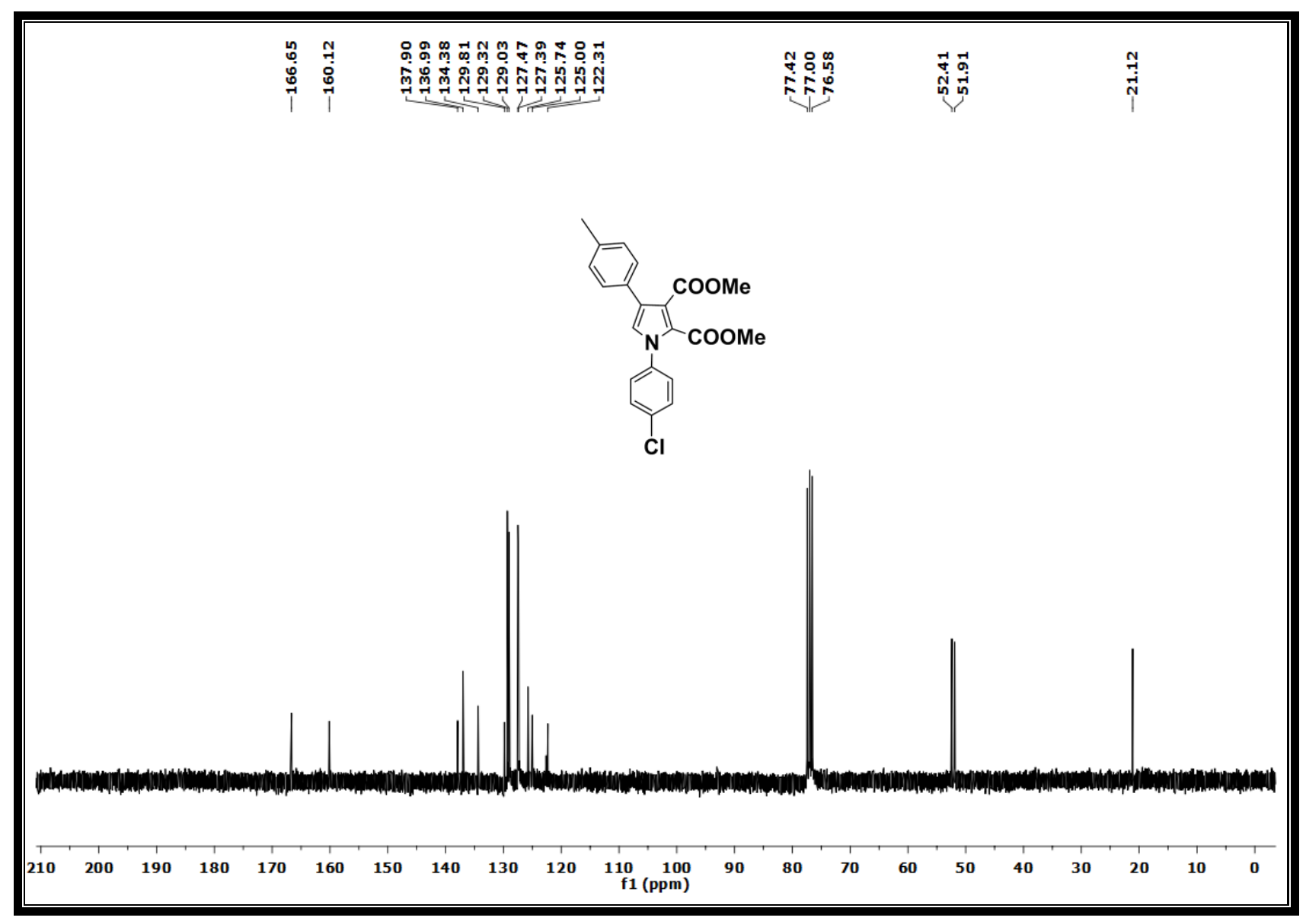

Fig. $45 .{ }^{13} \mathrm{C}$ NMR spectrum $3 t$ 


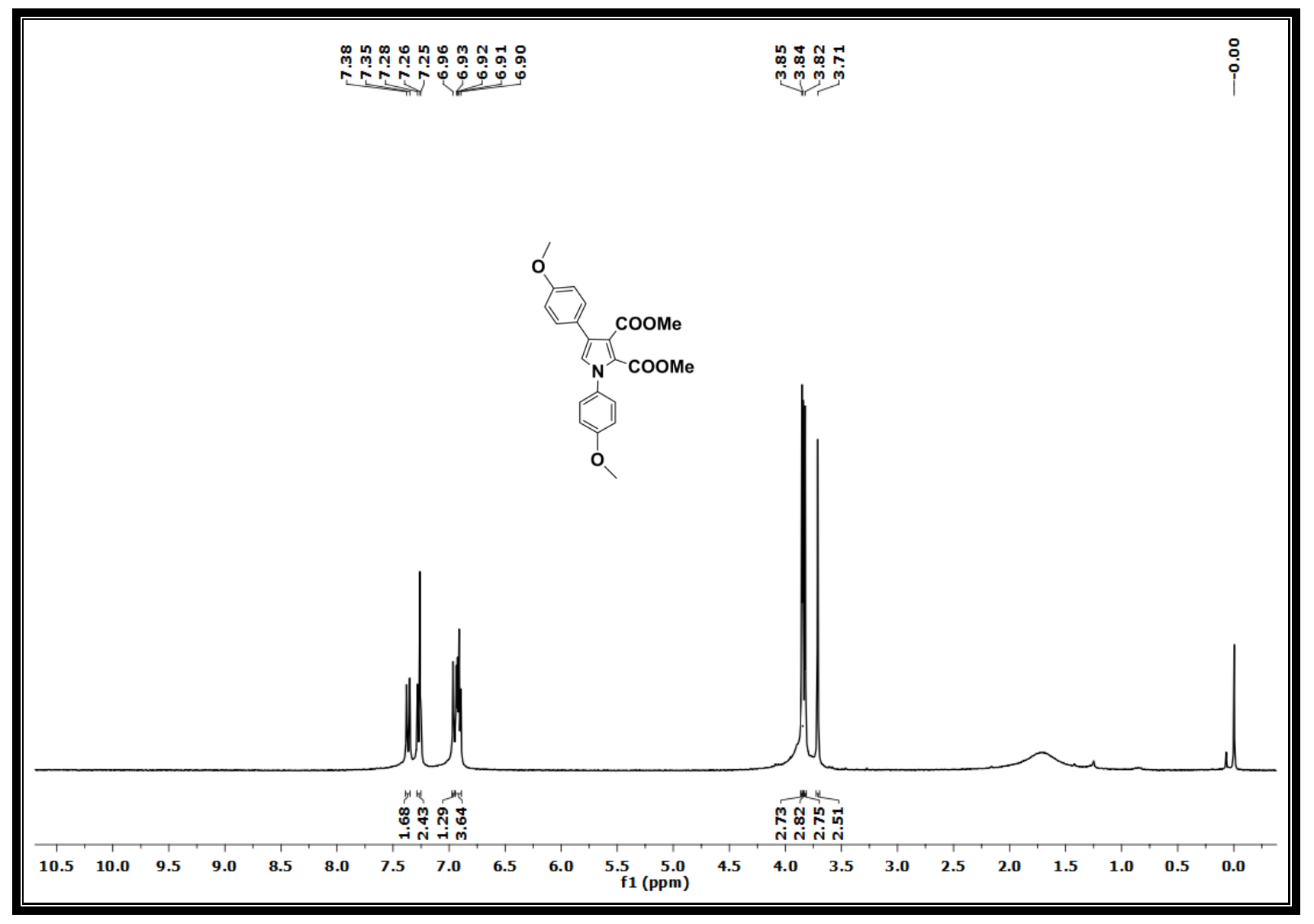

Fig.46. ${ }^{1} \mathrm{H}$ NMR spectrum $\mathbf{3 u}$ 


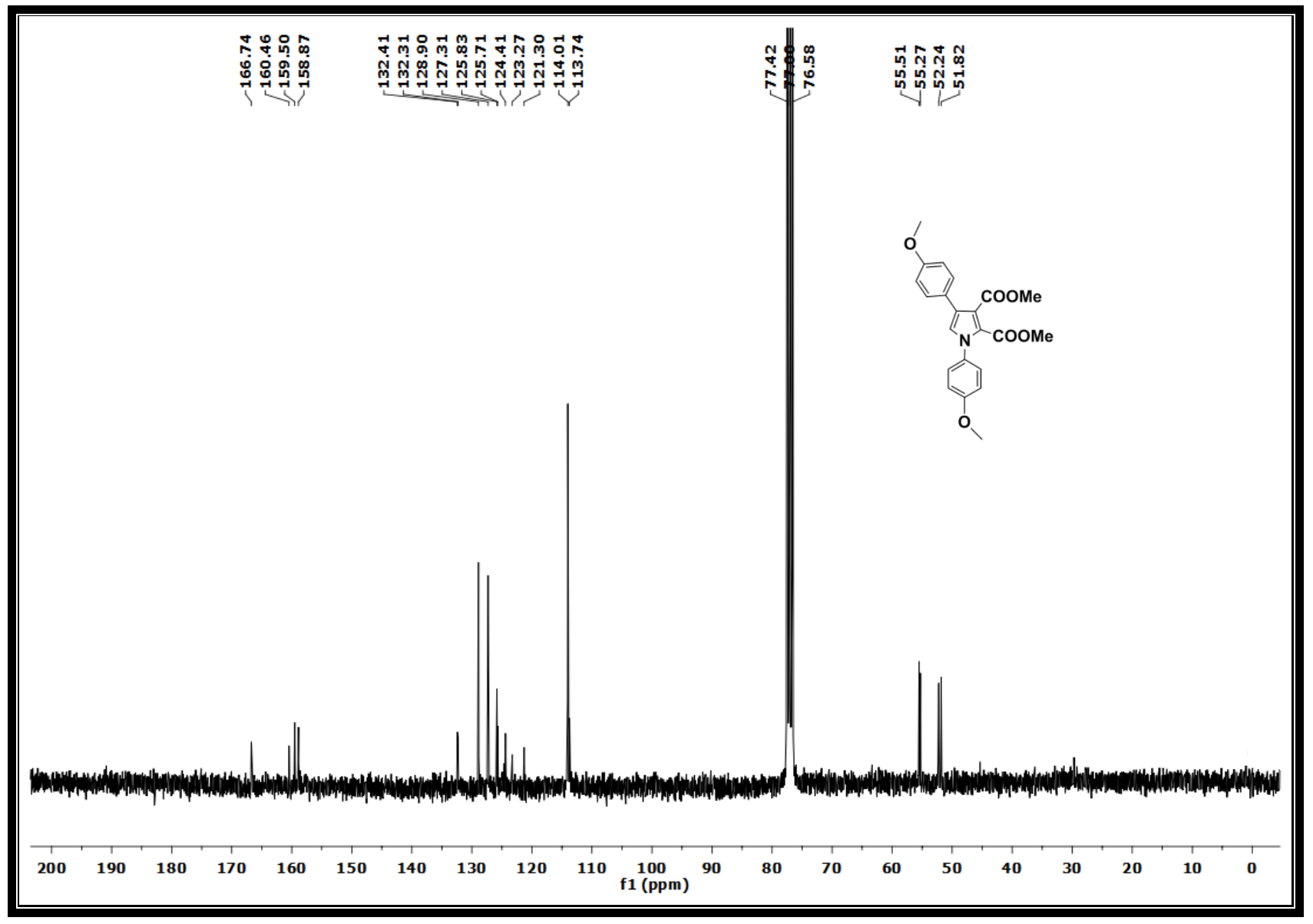

Fig.47. ${ }^{13} \mathrm{C}$ NMR spectrum $3 \mathbf{u}$ 


\section{REFERENCES}

1. Khetan, S.K.; Hiriyakkanavar, J.G.; George, M.V. Tetrahedron 1968, 24, 1567. 